\title{
The identification of DNA binding regions of the $\sigma 54$ factor using artificial neural network
}

\author{
FERREIRA, L. ${ }^{1}$; RAITTZ, R. T. ${ }^{1}$; MARCHAUKOSKI, J. N. ${ }^{1}$; WEISS, V. A. ${ }^{1}$; SANTOS-WIESS, I. C. R. ${ }^{1}$; COSTA, P. A. \\ B. ${ }^{1 ;}$ VOYCEIK, R. ${ }^{1}$; RIGO, L. U. ${ }^{2}$ \\ 1 - Laboratory of Bioinformatics, Federal University of Parana, Curitiba, Parana, Brazil; \\ 2 - Department of Biochemistry and Molecular Biology, Federal University of Parana, Curitiba, Parana, Brazil.
}

\begin{abstract}
Transcription of many bacterial genes is regulated by alternative RNA polymerase sigma factors as the sigma 54 ( $\sigma 54)$. A single essential $\sigma$ promotes transcription of thousands of genes and many alternative $\sigma$ factors promote transcription of multiple specialized genes required for coping with stress or development. Bacterial genomes have two families of sigma factors, sigma $70(\sigma 70)$ and sigma $54(\sigma 54)$. o54 uses a more complex mechanism with specialized enhancers-binding proteins and DNA melting and is well known for its role in regulation of nitrogen metabolism in proteobacteria. The identification of these regulatory elements is the main step to understand the metabolic networks. In this study, we propose a supervised pattern recognition model with neural network to identify Transcription Factor Binding Sites (TFBSs) for $\sigma 54$. This approach is capable of detecting $\sigma 54$ TFBSs with sensitivity higher than $98 \%$ in recent published data. False positives are reduced with the addition of ANN and feature extraction, which increase the specificity of the program. We also propose a free, fast and friendly tool for $\sigma 54$ recognition and a $\sigma 54$ related genes database, available for consult. S54Finder can analyze from short DNA sequences to complete genomes and is available online. The software was used to determine 654 TFBSs on the complete bacterial genomes database from NCBI and the result is available for comparison. S54Finder does the identification of $\sigma 54$ regulated genes for a large set of genomes allowing evolutionary and conservation studies of the regulation system between the organisms.
\end{abstract}

Keywords: Artificial intelligence, pattern recognition, feature extraction. 


\section{Background}

Prokaryotic transcription process starts from the recognition of binding site on DNA sequence by RNA polymerase. The DNA dependent RNA Polymerase (RNAP) is the main enzyme in the transcription and gene expression [1]. It is composed of two subunits, the core enzyme $\alpha 2 \beta \beta$ with the catalytic site of polymerization and the sigma factors, responsible for promoter recognition [1]. When the enzyme is linked with the sigma factor protein, the complex is called of RNAP holoenzyme $\left(\alpha 2 \beta \beta^{\prime} \sigma\right)$ and is ready for transcription [2].

Bacterial genomes have two families of sigma factors, sigma $70(\sigma 70)$ and sigma 54 ( $\sigma 54) . \sigma 70$ is the best studied and is responsible for transcription of the most bacterial genes in the exponential growth [3]. o54 uses a more complex mechanism with specialized enhancers-binding proteins and DNA melting and is well known for its role in regulation of nitrogen metabolism in proteobacteria [4].

Each sigma factor recognizes different binding sites in the DNA sequence and is responsible for changing the transcription pattern mediated thorough redirection of transcription initiation [5]. The identification of these regulatory elements is the main step to understand the metabolic networks [6].

A single essential $\sigma$ promotes transcription of thousands of genes and many alternative $\sigma$ factors promote transcription of multiple specialized genes required for coping with stress or development [7].

The number of sigma factors varies from one in Mycoplasma genitalia [8], seven in Escherichia coli [9] to more than 60 in Streptomyces coelicolor [10].

It was already shown that $\sigma 70$ recognize a consensus sequence of hexamers placed between -10/-35 nucleotides upstream the start site and o54 recognize high GC sequences located $-24 /-12$ nucleotides upstream from the start site [11].

The $\sigma 54$ factor has an important role in agriculture, due to the control of gene transcription, regulating the nitrogen fixation process that provides ammonia for the plant. This process dispenses chemical soil fertilization, reduces costs and protects the environment [12].

The classical approach for promoter prediction involves the development of algorithms that uses position weight matrices (PWMs) [13-17]. More recently, several methods propose the automatic identification of Transcription Factor Binding Sites (TFBSs) like Gibbs sampling algorithm [18, 19], genetic algorithms [20], and statistical overrepresentation [21, 22]. These methods works with single and double motifs identification but with a high false positive rate [23], and don't allow complete genome sequences.

In this study, we propose the use of a supervised pattern recognition model with neural network to identify TFBSs for $\sigma 54$ for whole genome sequence. The advantages of using neural network in pattern recognition include its generalization ability considering both the nonlinear and the diffuse nature of the datasets [24] and also can achieve high performance when processing extended genome sequences [25].

We also propose a free, fast and friendly tool for $\sigma 54$ recognition and a $\sigma 54$ database available for consult. 


\section{Methods}

\subsection{Candidates sequences for $\sigma 54$ TFBS and features extraction}

The set of intergenic regions to search for 054 TFBSs candidates was defined as a range of 200 bases from the transcriptional start site of each coding sequence. Candidate sequences were set on 16 bases. The selection was done considering the presence of conserved bases (GG and GC) located in -14/-15 and -2/-3 positions [11]. 18 features were extracted from the candidate sequences. The first sixteen were the nucleotides encoded to numbers (A: $0.0 ; \mathrm{C}: 1.0 ; \mathrm{G}: 2.0 ; \mathrm{T}: 3.0)$; the $17^{\text {th }}$ feature was the result of the alignment of the candidate sequence with the consensus sequence, extracted from experimentally confirmed sequences available in literature [11, 26, 27]; and the $18^{\text {th }}$ feature was the result of the alignment of the sequence candidate with a consensus of the less frequent bases (present in the true sequences- called as anticonsensus).

\subsection{Training data set}

Networks were trained using groups of positive sequences collected in the literature and sequences artificially generated, considered as negatives. Those sequences were generated using the MatLab algorithm of string generation. The artificially generated strings were divided into two groups, the first group have only nucleotide base pairs and the same string length of positive sequences. In other side the second group uses also the same length, but it also includes the conserved base pairs shown in the positive sequences.

Positive patterns were defined by sequences experimentally retrieved [26, 27] and a database with 5662 sequences from more than 60 genomes, available in www.sigma54.ca web site [28]. The artificial dataset comprised two groups of random sequences. The groups had the same number of bases observed in the positive pattern and differ by the presence and absence of the highly conserved bases ( $G G$ and $\mathrm{GC)}$.

\subsection{ANN test}

Tests with MLP (Multilayer perceptron), FAN (Free Associative Neurons), RBF (Radial Basis Function) and SVM (Support Vector Machine) neural networks were performed. RBF and SVM did not show better results than MLP and FAN neural networks. The best architecture used to classify the sequences using MLP neural network had 7 neurons in the first hidden layer and three neurons in the second one. For FAN neural network we used the default setting with fuzzy set support of 100 , diffuse neighboring of 6 , shuffling the training set every time. The accuracy for each neural network tested on the same database was $95.19 \%$ for MLP and $97.95 \%$ for the chosen one, the FAN network (Table 1).

Table 1. Performance comparison for neural networks.

\begin{tabular}{lcc}
\hline & MLP & FAN \\
\hline True positives, \% & 92.15 & $\mathbf{9 5 . 5 1}$ \\
False positives, \% & 3.29 & 2.09 \\
True negatives, \% & 96.71 & 97.91 \\
False negatives, \% & 7.85 & 4.49 \\
Accuracy, \% & 95.19 & $\mathbf{9 7 . 9 5}$ \\
\hline
\end{tabular}


The ANN test was performed using 3 datasets from published studies comprising 281 proposed 054 TFBS sequences that were not used for training (Table 2). For more recent data, the sensitivity test showed more than $98 \%$ of right predictions for 054 binding sites sequences. The decrease of sensitivity observed on Barrios database was due to the presence of candidate sequences in the $\sigma 54$ TFBS database.

Table 2. ANN sensitivity in comparison with published data.

\begin{tabular}{lccc}
\hline \multicolumn{1}{c}{ Dataset } & ANN Positive & Total & Sensitivity (\%) \\
\hline Studholme, 2000 [29] & 34 & 34 & 100 \\
Leang, 2009 [30] & 90 & 91 & 98.9 \\
Barrios, 1999 [11] & 127 & 156 & 81.4 \\
\hline
\end{tabular}

The sequences that did not had the conserved bases GG and GC and without known amino acids, were excluded from the test.

\subsection{S54Finder tool}

S54Finder is a fast tool developed to work with complete genome sequences. It works with annotated sequences stored in a gbk file format parsing the information to perform the analysis. If the query is a flat fasta file the software uses an in-house ORFinder to identify and annotate the coding regions and extract the intergenic sequences. The output of the S54Finder is 2 files, a new gbk file with the predicted $\sigma 54$ sequences marked and a text file with the predicted sequences and related ORFs positions to download.

\section{Results and discussion}

There is no TFBSs consensus for $\sigma 54$, with only few sequences confirmed in laboratory. Several studies present candidate sequences as consensus but the size usually is not the same, since it differs between species. What is observed is that among the vast majority of the sequences there are at least 4 (GG and GC) conserved bases. This scenario turns difficult the pattern recognition since it increases the false positive rate. To overcome this problem we added the anti-consensus feature, which allow us to decrease candidate number usually observed in other tools. To test S54Finder false positive rate we used $20^{12}$ sequences generated by combination of bases preserving the high conserved ones (GG and $G C$ ). The results showed that S54Finder identified, from the total of possibilities, $1.89 \%$ of $\sigma 54$ candidate sequences. This result confirms a high specificity of the S54Finder software.

We tried to compare S54Finder with other available tools such as GenomeMatScan, but the test was not possible since GenomeMatScan does not perform whole genome predictions. The main features between the two software applications are contrasted in Table 3.

Table 3. Comparison between S54Finder and GenomeMatScan software.

\begin{tabular}{lll}
\hline & S54Finder & GenomeMatScan \\
\hline Web version & Yes & Yes \\
Local processing & Yes & No \\
$\begin{array}{ll}\text { Method } \\
\text { Data entry }\end{array}$ & FAN & HMM \\
& Genbank file (.gbk) & $\begin{array}{l}\text { Input data copy-pasted into the } \\
\text { browser }\end{array}$ \\
Results & Output in Genbank formats (.gbk), & Shown in browser \\
& MatLab (.mat) and text & No \\
$\begin{array}{l}\text { Processing large } \\
\text { regions of DNA }\end{array}$ & Yes (complete genomes) & \\
\hline HMM: Hidden Markov Models.
\end{tabular}


The distinguish feature of S54Finder is the ability to perform fast whole genome predictions. This allowed us to search for TFBSs in complete bacterial genomes across the entire $\mathrm{NCBI}$ database. From the complete genomes, s54finder obtained 79626 CDS sequences which could be ranked by the level of TFBSs occurrence next to homologous genes of different species.

An in house tool was employed to cluster the CDS. A set of 9417 homologous CDS groups, within 2 or more homologous occurrences were determined. The minimal relative score considered to group a pair of genes was 0.5 . To determine the annotation to be considered for each gene cluster we implemented a kmeans to support the choice in the set of original annotations. The generated data TFBSs, CDSs, annotation, Homologous clusters - were gathered to perform a FASTA/BLAST formatted data base available and a web site (http://200.236.3.16/s54.php), that is available to validate the S54Finder predictions. Table 4 presents a list with the 29 groups that occurred 100 or more times. The glutamine synthetase protein and the nitrogen regulatory protein P II appears in the top of the list with 379 and 329 occurrences, respectively. Due to the importance of these proteins in the biologic nitrogen fixation their presence in the top predicted list was expected. We proposed a score to determine the correlation of a word present in an $\mathrm{NCBI}$ annotation found by s54finde with conserved clusters of genes related to TFBS. A binary matrix where columns represent the occurrence of each word and the lines the homologous groups, was joined to a column containing the number of occurrences of the respective homologous group, $\mathrm{T}$. The ws54score of a word is then the Pearson correlation between the column that represent the word and homologous amount column, T. Our purpose was to identify possibly related words to $\sigma 54$ regulated genes. Highest ws54scores were associated to gene names like glnL (0.3711), glnB (0.2959), glnE (0.2959) and PII (0.2959), whose association with the $\sigma 54$ promoter is well known. However, words like MtrA and Thi1, also appeared in the top 10 list though not common in the literature; unknown conserved domains also appeared - DUF2233 (4) and DUF2971 (11) - in the top 15 list and we suppose they are likely related to the $\sigma 54$. The list with annotation words and s54scores is in the Additional File.

\section{Conclusion}

The S54Finder presents good results regarding research in bacterial genomes. The main advantage is the fact that it is faster than other available tools. The pre-selection of candidates without the use of neural networks has shown that the pattern of conservation, based on published sequences and biologically confirmed, is capable of locating binding sites in bacterial genomes, but generates many false positives.

False positives are reduced with the addition of ANN and feature extraction, which increase the specificity of the program. S54Finder allows the user the identification of o54-regulated genes for a large set of genomes allowing evolutionary and conservation studies of the regulation system between the organisms. The tool and the o54 database are freely available in web.

\section{Authors' contributions}

Following are the contributions of the authors to the work described in this paper. LMF: acquisition of data; analysis and interpretation of data; RTR: made substantial contributions to conception and design of the study; analysis and interpretation of data; JNM: have been involved in drafting the manuscript or revising it critically for important intellectual content; have given final approval of the version to be published; VAW: have been involved in drafting the manuscript or revising it critically for important intellectual content; have given final approval of the version to be published; ICRSW: 
bioRxiv preprint doi: https://doi.org/10.1101/393736; this version posted August 17, 2018. The copyright holder for this preprint (which was not certified by peer review) is the author/funder, who has granted bioRxiv a license to display the preprint in perpetuity. It is made available under aCC-BY-NC-ND 4.0 International license.

have been involved in drafting the manuscript or revising it critically for important intellectual content; have given final approval of the version to be published; PAB: analysis and interpretation of data; RV: participated in the website development and revising the manuscript; LUR: made substantial contributions to conception and design of the study. All authors read and approved the final manuscript.

\section{Acknowledgements}

\section{CNPq (Conselho Nacional de Desenvolvimento Científico e Tecnológico) and CAPES (Coordenação de Aperfeiçoamento de Pessoal de Nível Superior) supported this work.}

\section{References}

[1] Gallegos MT, Cannon WV, Buck M. Functions of the sigma (54) region I in trans and implications for transcription activation. J Biol Chem. 1999; 274(36):25285-90.

[2] Murakami KS, Darst SA. Bacterial RNA polymerases: the whole story. Curr Opin Struct Biol. 2003; 13(1):31-9.

[3] Lonetto M, Gribskov M, Gross CA. The sigma 70 family: sequence conservation and evolutionary relationships. J Bacteriol. 1992; 174 (12):3843-9.

[4] Buck M, Gallegos M-T, Studholme DJ, Guo Y, Gralla JD. The Bacterial Enhancer-Dependent $\varsigma 54(\varsigma N)$ Transcription Factor. J Bacteriol. 2000; 182(15):4129-36.

[5] Salzberg SL, Delcher AL, Kasif S, White O. Microbial gene identification using interpolated Markov models. Nucleic Acids Res. 1998; 26(2):544-8.

[6] Tompa M, Li N, Bailey TL, Church GM, De Moor B, Eskin E, Favorov AV, Frith MC, Fu Y, Kent WJ, Makeev VJ, Mironov AA, Noble WS, Pavesi G, Pesole G, Régnier M, Simonis N, Sinha S, Thijs G, van Helden J, Vandenbogaert M, Weng Z, Workman C, Ye C, Zhu Z. Assessing computational tools for the discovery of transcription factor binding sites. Nat Biotechnol. 2005; 23(1):137-44.

[7] Feklistov A, Sharon BD, Darst SA, Gross CA. Bacterial Sigma Factors: A Historical, Structural, and Genomic Perspective. Annu Rev Microbiol. 2014; 68: 357-376.

[8] Fraser CM, Gocayne JD, White O, Adams MD, Clayton RA, Fleischmann RD, Bult CJ, Kerlavage AR, Sutton G, Kelley JM, Fritchman RD, Weidman JF, Small KV, Sandusky M, Fuhrmann J, Nguyen D, Utterback TR, Saudek DM, Phillips CA, Merrick JM, Tomb JF, Dougherty BA, Bott KF, Hu PC, Lucier TS, Peterson SN, Smith HO, Hutchison CA 3rd, Venter JC. The minimal gene complement of Mycoplasma genitalium. Science, 1995; 270: 397-403.

[9] Ishihama A. Functional modulation of Escherichia coli RNA polymerase. Annu Rev Microbiol., 2000; 54: $499-518$. [10] Bentley SD, Chater KF, Cerdeno-Tarraga AM, Challis GL, Thomson NR, James KD, Harris DE, Quail MA, Kieser H, Harper D, Bateman A, Brown S, Chandra G, Chen CW, Collins M, Cronin A, Fraser A, Goble A, Hidalgo J, Hornsby T, Howarth S, Huang CH, Kieser T, Larke L, Murphy L, Oliver K, O'Neil S, Rabbinowitsch E, Rajandream MA, Rutherford K, Rutter S, Seeger K, Saunders D, Sharp S, Squares R, Squares S, Taylor K, Warren T, Wietzorrek A, Woodward J, Barrell BG, Parkhill J, Hopwood DA. Complete genome sequence of the model actinomycete Streptomyces coelicolor A3(2). Nature, 2002; 417: 141-147.

[11] Barrios H, Valderrama B, Morett, E. Compilation and analysis of s54-dependent promoter sequences. Nucleic Acids Res., 1999; 27: 4305-4313.

[12] Postgate JR. Biological nitrogen fixation: Fundamental. Phil. Trans. R. Soc. Lond., 1982; 296: 375-85.

[13] Li QZ, Lin H. The recognition and prediction of s70 promoters in Escherichia coli K-12. Journal of Theoretical Biology. 2006; 242: 135-141.

[14] Tsunoda T, Takagi T. Estimating transcription factor bindability on DNA. Bioinformatics, 1999; 15(7):622-30.

[15] Messeguer X, Escudero R, Farré D, Núñez O, Martínez J, Albà MM. PROMO: detection of known transcription regulatory elements using species-tailored searches. Bioinformatics, 2002; 18(2):333-4.

[16] Mathelier A, Zhao X, Zhang AW, Parcy F, Worsley-Hunt R, Arenillas DJ, Buchman S, Chen CY, Chou A, lenasescu H, Lim J, Shyr C, Tan G, Zhou M, Lenhard B, Sandelin A, Wasserman WW. JASPAR 2014: an extensively expanded and updated open-access database of transcription factor binding profiles. Nucleic Acids Res. 2014; 42: D142-7.

[17] Cartharius K, Frech K, Grote K, Klocke B, Haltmeier M, Klingenhoff A, Frisch M, Bayerlein M, Werner T. MatInspector and beyond: promoter analysis based on transcription factor binding sites. Bioinformatics, 2005; 21(13):2933-42.

[18] Frith MC, Hansen U, Spouge JL, Weng Z. Finding functional sequence elements by multiple local alignment. Nucleic Acids Res., 2004; 32(1):189-200.

[19] Roth FP, Hughes JD, Estep PW, Church GM. Finding DNA regulatory motifs within unaligned noncoding sequences clustered by whole-genome mRNA quantitation. Nat Biotechnol., 1998; 16(10):939-45.

[20] Gertz J, Riles L, Turnbaugh P, Ho S-W, Cohen BA. Discovery, validation, and genetic dissection of transcription factor binding sites by comparative and functional genomics. Genome Res., 2005; 15(8):1145-52.

[21] Van Helden J, André B, Collado-Vides J. Extracting regulatory sites from the upstream region of yeast genes by computational analysis of oligonucleotide frequencies. J Mol Biol., 1998; 281(5):827-42.

[22] Touzain F, Schbath S, Debled-Rennesson I, Aigle B, Kucherov G, Leblond P. SIGffRid: A tool to search for sigma factor binding sites in bacterial genomes using comparative approach and biologically driven statistics. BMC Bioinformatics, 2008; 9(1):73.

[23] de Avila e Silva S, Gerhardt GJ, Echeverrigaray S. Rule's extraction from neural networks applied to the prediction and recognition of prokaryotic promoters. 2011;34: 353-360.

[24] Lakshmi KV, Padmavathamma M. Modeling an Expert System for Diagnosis of Gestational Diabetes Mellitus Based On Risk Factors. J Computer Eng (IOSRJCE), 2013; 8: 29-32.

[25] Cotik, V, Zaliz, RR, Zwir, I. A hybrid promoter analysis methodology for prokaryotic genomes. Fuzzy Sets and Systems. 2005; 1:83-102. 
bioRxiv preprint doi: https://doi.org/10.1101/393736; this version posted August 17, 2018. The copyright holder for this preprint (which was

not certified by peer review) is the author/funder, who has granted bioRxiv a license to display the preprint in perpetuity. It is made available under aCC-BY-NC-ND 4.0 International license.

[26] Souza JA. Reconhecimento de padrões usando indexação recursiva. Tese de Doutorado, Universidade Federal de Santa Catarina, 1999.

[27] Schwab S, Souza EM, Yates MG, Persuhn DC, Steffens MBR, Chubatsu L S, Pedrosa FO, Rigo LU. The gInAntrBC operon of Herbaspirillum seropedicae is transcribed by two oppositely regulated promoters upstream of glnA. Can. J. Microbiol., 2007; 53: 100-105.

[28] Conway K. s54 Promoter data base. http://www.sigma54.ca/PromoterApp/Web/data.aspx. Accessed: 16 Feb 2012. [29] Studholme DJ, Wigneshwereraraj SR, Gallegos MT, Buck M. Functionality of Purified sN (s54) and a NifA-Like Protein from the Hyperthermophile Aquifex aeolicus. J Bacteriol. 2000; 182 (6): 1616-1623.

[30] Leang C. Krushkal J, Ueki T, Puljic M, Sun J, Juárez K, Núñez C, Reguera G, Didonato R, Postier B, Adkins RM, Lovley DR. Genome-wide analysis of the RpoN regulon in Geobacter sulfurreducens. BMC Genomics. 2009; 10: 331. 


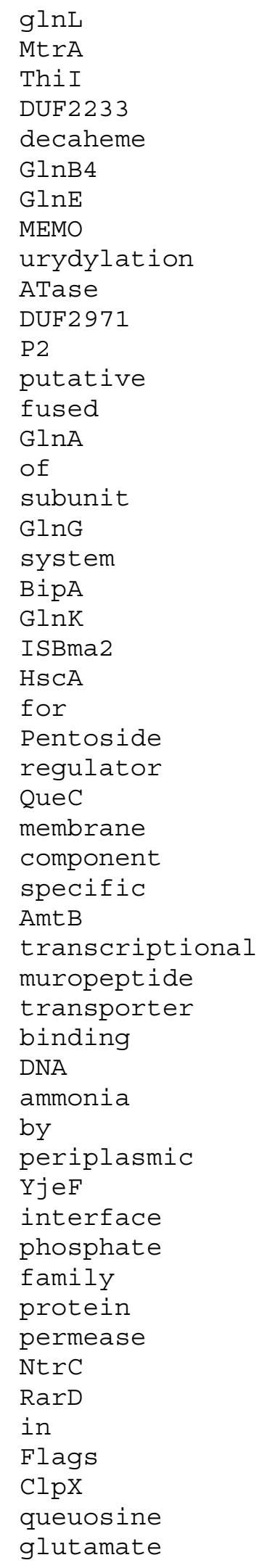




\begin{tabular}{|c|c|c|}
\hline 53 & unknown & 0.18234 \\
\hline 54 & type & 0.18205 \\
\hline 55 & dependent & 0.182 \\
\hline 56 & function & 0.18026 \\
\hline 57 & SufB & 0.17839 \\
\hline 58 & xanthine & 0.17827 \\
\hline 59 & diffusion & 0.17625 \\
\hline 60 & murein & 0.17586 \\
\hline 61 & Empty & 0.17556 \\
\hline 62 & dehydrogenase & 0.17545 \\
\hline 63 & galaxtoside & 0.17522 \\
\hline 64 & connecting & 0.17522 \\
\hline 65 & $\mathrm{HBP}$ & 0.17522 \\
\hline 66 & $\mathrm{PdxB}$ & 0.17522 \\
\hline 67 & locus & 0.17241 \\
\hline 68 & $A B C$ & 0.17221 \\
\hline 69 & biosynthesis & 0.17205 \\
\hline 70 & pectin & 0.17205 \\
\hline 71 & polar & 0.17027 \\
\hline 72 & inner & 0.17015 \\
\hline 73 & like & 0.17004 \\
\hline 74 & $\mathrm{GlnB}$ & 0.16994 \\
\hline 75 & kinase & 0.1689 \\
\hline 76 & PAP2 & 0.16888 \\
\hline 77 & acid & 0.16851 \\
\hline 78 & Hexuronide & 0.1685 \\
\hline 79 & tricarboxylate & 0.1683 \\
\hline 80 & PTS & 0.16826 \\
\hline 81 & PadR & 0.16758 \\
\hline 82 & RecG & 0.16667 \\
\hline 83 & sensing & 0.16576 \\
\hline 84 & III & 0.16425 \\
\hline 85 & YVTN & 0.16424 \\
\hline 86 & ExaC & 0.16424 \\
\hline 87 & achromobactin & 0.16424 \\
\hline 88 & $\mathrm{XkdN}$ & 0.16424 \\
\hline 89 & $\operatorname{Tct} A$ & 0.16424 \\
\hline 90 & synthetase & 0.16413 \\
\hline 91 & formation & 0.16385 \\
\hline 92 & sigma & 0.16298 \\
\hline 93 & and & 0.16239 \\
\hline 94 & $20 \mathrm{~S}$ & 0.16059 \\
\hline 95 & nitrogen & 0.1603 \\
\hline 96 & GTP & 0.15945 \\
\hline 97 & Predicted & 0.15833 \\
\hline 98 & portion & 0.15793 \\
\hline 99 & glucitol & 0.15752 \\
\hline 100 & II & 0.15708 \\
\hline 101 & NR & 0.15673 \\
\hline 102 & reductase & 0.15659 \\
\hline 103 & arsenate & 0.1557 \\
\hline 104 & acetylmuramoyl & 0.15565 \\
\hline 105 & FhlA & 0.1541 \\
\hline 106 & ТурА & 0.15374 \\
\hline
\end{tabular}




\begin{tabular}{|c|c|}
\hline 107 & assimilatory \\
\hline 108 & hydrolase \\
\hline 109 & Tyr \\
\hline 110 & chain \\
\hline 111 & guanosine \\
\hline 112 & RNA \\
\hline 113 & CsiR \\
\hline 114 & superfamily \\
\hline 115 & LivK \\
\hline 116 & NS2 \\
\hline 117 & factor \\
\hline 118 & beta \\
\hline 119 & diaminohydroxyphosphoribosylaminopyrimidine \\
\hline 120 & FeS \\
\hline 121 & gamma \\
\hline 122 & protoporphyrin \\
\hline 123 & camphor \\
\hline 124 & nucleotide \\
\hline 125 & $\mathrm{ATP}$ \\
\hline 126 & modulated \\
\hline 127 & ISSfl2 \\
\hline 128 & into \\
\hline 129 & coproporphyrinogen \\
\hline 130 & AsmA \\
\hline 131 & IIBC \\
\hline 132 & ribosomal \\
\hline 133 & styLTI \\
\hline 134 & PrpR \\
\hline 135 & PrpB \\
\hline 136 & repressor \\
\hline 137 & $\operatorname{CrcB}$ \\
\hline 138 & efflux \\
\hline 139 & Rets \\
\hline 140 & IS1665 \\
\hline 141 & UrtC \\
\hline 142 & UrtE \\
\hline 143 & uera \\
\hline 144 & AmiC \\
\hline 145 & $\mathrm{TTT}$ \\
\hline 146 & invasin \\
\hline 147 & La \\
\hline 148 & signal \\
\hline 149 & Precursor \\
\hline 150 & activase \\
\hline 151 & carboxypeptidase \\
\hline 152 & $\mathrm{TC}$ \\
\hline 153 & multidrug \\
\hline 154 & IS902 \\
\hline 155 & IS116 \\
\hline 156 & 54 \\
\hline 157 & operon \\
\hline 158 & interaction \\
\hline 159 & bound \\
\hline 160 & lyase \\
\hline
\end{tabular}

0.15368

0.15311

0.15262

0.15088

0.15075

0.15043

0.14888

0.14845

0.14829

0.14758

0.14732

0.14723

0.14694

0.14671

0.14658

0.14647

0.14609

0.14574

0.14555

0.14538

0.145

0.145

0.14478

0.14478

0.14426

0.14386

0.14321

0.14321

0.14321

0.14184

0.141

0.14097

0.14047

0.14047

0.14047

0.14047

0.14047

0.14047

0.14047

0.13984

0.1396

0.13906

0.13905

0.13903

0.138

0.13733

0.13704

0.13694

0.13694

0.13639

0.13596

0.13523

0.13383

0.13326 


\begin{tabular}{|c|c|}
\hline 161 & synthase \\
\hline 162 & EIIB \\
\hline 163 & transaldolase \\
\hline 164 & Lysine \\
\hline 165 & chb \\
\hline 166 & chitobiose \\
\hline 167 & YeaH \\
\hline 168 & AstC \\
\hline 169 & ArgM \\
\hline 170 & pentulose \\
\hline 171 & tyrosyl \\
\hline 172 & Two \\
\hline 173 & Ntr \\
\hline 174 & alpha \\
\hline 175 & Ile \\
\hline 176 & chelatase \\
\hline 177 & Fis \\
\hline 178 & sigma 54 \\
\hline 179 & accessory \\
\hline 180 & resistance \\
\hline 181 & thioesterase \\
\hline 182 & ArgT \\
\hline 183 & $\mathrm{HU}$ \\
\hline 184 & containing \\
\hline 185 & val \\
\hline 186 & leu \\
\hline 187 & arginine \\
\hline 188 & succunate \\
\hline 189 & CoxI \\
\hline 190 & PhoQ \\
\hline 191 & nifK \\
\hline 192 & $\mathrm{NifH} 2$ \\
\hline 193 & nifD \\
\hline 194 & DedA \\
\hline 195 & with \\
\hline 196 & racemase \\
\hline 197 & lytic \\
\hline 198 & acetaldehyde \\
\hline 199 & oligosaccharide \\
\hline 200 & salt \\
\hline 201 & CelA \\
\hline 202 & YeaG \\
\hline 203 & autokinase \\
\hline 204 & LicB \\
\hline 205 & TrmB \\
\hline 206 & sulfur \\
\hline 207 & transmembrane \\
\hline 208 & IIA \\
\hline 209 & oxidoreductase \\
\hline 210 & the \\
\hline 211 & amide \\
\hline 212 & residue \\
\hline 213 & expression \\
\hline 214 & IS110 \\
\hline
\end{tabular}
0.13302
0.13298
0.13284
0.13231
0.13224
0.13224
0.13224
0.13224
0.13224
0.13224
0.1317
0.1316
0.13142
0.13059
0.13039
0.12978
0.12977
0.1296
0.12953
0.12938
0.12915
0.12883
0.12872
0.12778
0.12735
0.12735
0.12685
0.12675
0.12675
0.12675
0.12675
0.12675
0.12675
0.12648
0.12616
0.12577
0.12495
0.12453
0.12401
0.12401
0.12401
0.12401
0.12401
0.12401
0.12401
0.12294
0.12257
0.1224
0.12235
0.12145
0.12137
0.12107
0.12063
0.12063 


\begin{tabular}{|c|c|}
\hline 215 & glucan \\
\hline 216 & $\mathrm{Mg}$ \\
\hline 217 & HypA \\
\hline 218 & IIC \\
\hline 219 & CheR \\
\hline 220 & enzyme \\
\hline 221 & isoenzymes \\
\hline 222 & NtrB \\
\hline 223 & domain \\
\hline 224 & outer \\
\hline 225 & protease \\
\hline 226 & RbsR \\
\hline 227 & inversion \\
\hline 228 & HBsu \\
\hline 229 & HupB \\
\hline 230 & DUF1481 \\
\hline 231 & aminotransferase \\
\hline 232 & Zras \\
\hline 233 & ZraR \\
\hline 234 & alternative \\
\hline 235 & nitrite \\
\hline 236 & histidine \\
\hline 237 & Methyltransferase \\
\hline 238 & ligase \\
\hline 239 & Peptidase \\
\hline 240 & sensitivity \\
\hline 241 & terminal \\
\hline 242 & $\operatorname{Ssn} A$ \\
\hline 243 & selenate \\
\hline 244 & YgfK \\
\hline 245 & YgfM \\
\hline 246 & isomerase \\
\hline 247 & $\mathrm{NifA}$ \\
\hline 248 & polymerase \\
\hline 249 & L2 0 \\
\hline 250 & orf \\
\hline 251 & $\mathrm{NifB}$ \\
\hline 252 & LivJ \\
\hline 253 & Meso \\
\hline 254 & deacylase \\
\hline 255 & F33 \\
\hline 256 & ftsX \\
\hline 257 & ybl142 \\
\hline 258 & purH \\
\hline 259 & HisQ \\
\hline 260 & amino \\
\hline 261 & mannitol \\
\hline 262 & SrlR \\
\hline 263 & NorV \\
\hline 264 & NorR \\
\hline 265 & FlRd \\
\hline 266 & AexT \\
\hline 267 & TTSS \\
\hline 268 & prow \\
\hline
\end{tabular}
0.12051
0.12051
0.1203
0.11999
0.11989
0.11979
0.11913
0.11898
0.11895
0.11894
0.11873
0.11852
0.11852
0.11852
0.11852
0.11852
0.11816
0.11784
0.11784
0.11767
0.11767
0.11719
0.11677
0.11673
0.11637
0.11629
0.11584
0.11578
0.11578
0.11578
0.11578
0.11561
0.11526
0.11487
0.11461
0.11457
0.1145
0.11396
0.11396
0.11343
0.11304
0.11304
0.11304
0.11304
0.11292
0.11279
0.1124
0.11212
0.11212
0.11212
0.11212
0.11212
0.11212
0.11212 


\begin{tabular}{|c|c|}
\hline 269 & $\mathrm{CP}$ \\
\hline 270 & iron \\
\hline 271 & mandelate \\
\hline 272 & DUF 400 \\
\hline 273 & small \\
\hline 274 & muconate \\
\hline 275 & PII \\
\hline 276 & phenylalanyl \\
\hline 277 & Organism \\
\hline 278 & translocation \\
\hline 279 & tripartite \\
\hline 280 & high \\
\hline 281 & cation \\
\hline 282 & $\mathrm{NAD}$ \\
\hline 283 & lipopolysaccharide \\
\hline 284 & $\mathrm{HAAT}$ \\
\hline 285 & manno \\
\hline 286 & transglycosylase \\
\hline 287 & Response \\
\hline 288 & UDP \\
\hline 289 & cysQ \\
\hline 290 & DUF1107 \\
\hline 291 & Paps \\
\hline 292 & amidophosphoribosyltransferase \\
\hline 293 & StpA \\
\hline 294 & COG1238 \\
\hline 295 & HybF \\
\hline 296 & Нурв \\
\hline 297 & HypE \\
\hline 298 & Willebrand \\
\hline 299 & von \\
\hline 300 & pyridoxal \\
\hline 301 & substances \\
\hline 302 & $\bmod$ \\
\hline 303 & insertion \\
\hline 304 & fructose \\
\hline 305 & lipoprotein \\
\hline 306 & molybdochelatase \\
\hline 307 & processing \\
\hline 308 & $\mathrm{Cbl}$ \\
\hline 309 & $\mathrm{NaC}$ \\
\hline 310 & yersiniabactin \\
\hline 311 & $933 \mathrm{U}$ \\
\hline 312 & irp3 \\
\hline 313 & UV \\
\hline 314 & complex \\
\hline 315 & decarboxylase \\
\hline 316 & $\mathrm{Fe}$ \\
\hline 317 & HisJ \\
\hline 318 & hydrogenase \\
\hline 319 & MltA \\
\hline 320 & ubiquinol \\
\hline 321 & HyсA \\
\hline 322 & glycyl \\
\hline
\end{tabular}
0.11202
0.11182
0.11179
0.11137
0.11101
0.10996
0.10996
0.10953
0.10922
0.10883
0.10869
0.10853
0.10845
0.10844
0.10815
0.10814
0.10813
0.10813
0.108
0.1076
0.10755
0.10755
0.10755
0.10744
0.10664
0.10664
0.10664
0.10664
0.10664
0.10661
0.10661
0.10577
0.10556
0.10556
0.10553
0.10544
0.10518
0.10501
0.10485
0.10481
0.10481
0.10481
0.10481
0.10481
0.10481
0.10458
0.10394
0.10375
0.10341
0.10329
0.10311
0.10234
0.1022
0.10204 


\begin{tabular}{|c|c|}
\hline 323 & Cyclopropane \\
\hline 324 & assembly \\
\hline 325 & tRNA \\
\hline 326 & guanine \\
\hline 327 & substrate \\
\hline 328 & incorporation \\
\hline 329 & acyl \\
\hline 330 & MipA \\
\hline 331 & helicase \\
\hline 332 & sugar \\
\hline 333 & fixation \\
\hline 334 & hydroxylase \\
\hline 335 & ferredoxin \\
\hline 336 & Glc \\
\hline 337 & CsgA \\
\hline 338 & crl \\
\hline 339 & Curlin \\
\hline 340 & FrsA \\
\hline 341 & fibers \\
\hline 342 & binds \\
\hline 343 & YafO \\
\hline 344 & YafN \\
\hline 345 & holoenzyme \\
\hline 346 & yfed \\
\hline 347 & HycC \\
\hline 348 & hyc \\
\hline 349 & hyp \\
\hline 350 & porin \\
\hline 351 & magnesium \\
\hline 352 & sensor \\
\hline 353 & pyrazinamidase \\
\hline 354 & Glu \\
\hline 355 & Asp \\
\hline 356 & COA \\
\hline 357 & ligand \\
\hline 358 & cysteinyl \\
\hline 359 & associated \\
\hline 360 & $\mathrm{Clp}$ \\
\hline 361 & NADP \\
\hline 362 & CheV \\
\hline 363 & cyclase \\
\hline 364 & cyclic \\
\hline 365 & $\mathrm{NifH}$ \\
\hline 366 & DAACS \\
\hline 367 & MinE \\
\hline 368 & affinity \\
\hline 369 & MprA \\
\hline 370 & proximal \\
\hline 371 & IIB \\
\hline 372 & amidase \\
\hline 373 & ATPases \\
\hline 374 & AldA \\
\hline 375 & propeptide \\
\hline 376 & ethanol \\
\hline
\end{tabular}

0.10187
0.10182
0.10177
0.10167
0.10143
0.10004
0.10003

0.099719

0.099151

0.098995

0.098907

0.09865

0.098486

0.098409

0.098409

0.098409

0.098409

0.098409

0.098409

0.098409

0.098409

0.098409

0.098409

0.098409

0.098409

0.098409

0.098409

0.098301

0.097265

0.097238

0.097079

0.097058

0.096371

0.096284

0.096283

0.095877

0.095662

0.095318

0.095269

0.09521

0.095006

0.09496

0.094563

0.094563

0.094563

0.094501

0.093917

0.093712

0.093647

0.093611

0.093548

0.092855

0.092855

0.092646 


\begin{tabular}{|c|c|}
\hline 377 & polyprenylphenol \\
\hline 378 & lactonizing \\
\hline 379 & 40 \\
\hline 380 & $\mathrm{XdhC}$ \\
\hline 381 & ornithine \\
\hline 382 & FMN \\
\hline 383 & conserved \\
\hline 384 & thiamine \\
\hline 385 & methionyl \\
\hline 386 & $\mathrm{~b} 12$ \\
\hline 387 & dual \\
\hline 388 & L10 \\
\hline 389 & sorbitol \\
\hline 390 & effector \\
\hline 391 & aspartate \\
\hline 392 & antitermination \\
\hline 393 & Glycine \\
\hline 394 & Adenylosuccinate \\
\hline 395 & fimbrial \\
\hline 396 & novel \\
\hline 397 & RutR \\
\hline 398 & $\mathrm{YCCE}$ \\
\hline 399 & YcdM \\
\hline 400 & $\mathrm{Rh}$ \\
\hline 401 & Amt \\
\hline 402 & discriminating \\
\hline 403 & $\mathrm{RpoH}$ \\
\hline 404 & desulfhydrase \\
\hline 405 & LysR \\
\hline 406 & malate \\
\hline 407 & multifunctional \\
\hline 408 & CjaA \\
\hline 409 & FliY \\
\hline 410 & 27133 \\
\hline 411 & $\mathrm{EC}$ \\
\hline 412 & HydG \\
\hline 413 & $\mathrm{HdfR}$ \\
\hline 414 & DUF3225 \\
\hline 415 & RND \\
\hline 416 & exporter \\
\hline 417 & Nass \\
\hline 418 & NasF \\
\hline 419 & InvG \\
\hline 420 & cis \\
\hline 421 & Iichenan \\
\hline 422 & pyruvate \\
\hline 423 & deoxy \\
\hline 424 & NifS \\
\hline 425 & FliA \\
\hline 426 & glucosidase \\
\hline 427 & $\mathrm{XTP}$ \\
\hline 428 & dITP \\
\hline 429 & catabolite \\
\hline 430 & NifQ \\
\hline
\end{tabular}
0.092432
0.092399
0.092327
0.092136
0.092064
0.091958
0.091956
0.091892
0.091878
0.091741
0.091679
0.091667
0.091667
0.091637
0.091519
0.091504
0.091475
0.091279
0.091133
0.091094
0.091094
0.091094
0.091094
0.091094
0.091094
0.091094
0.090743
0.090472
0.090343
0.090256
0.090215
0.090179
0.090179
0.090179
0.090179
0.090179
0.090179
0.090179
0.089732
0.089548
0.089265
0.089265
0.089265
0.089141
0.088744
0.088577
0.088574
0.088478
0.088395
0.08764
0.087451
0.087451
0.08738
0.087106 


\begin{tabular}{|c|c|c|}
\hline 431 & Rlu & 0.086522 \\
\hline 432 & BztA & 0.086522 \\
\hline 433 & yhdW & 0.086522 \\
\hline 434 & AcrE & 0.086522 \\
\hline 435 & AapJ & 0.086522 \\
\hline 436 & axial & 0.086522 \\
\hline 437 & Era & 0.08652 \\
\hline 438 & Flagellar & 0.086266 \\
\hline 439 & transferase & 0.086115 \\
\hline 440 & transduction & 0.085925 \\
\hline 441 & machinery & 0.085511 \\
\hline 442 & loop & 0.085464 \\
\hline 443 & nitric & 0.085282 \\
\hline 444 & flhb & 0.084481 \\
\hline 445 & elongation & 0.08441 \\
\hline 446 & cell & 0.084229 \\
\hline 447 & nif & 0.083758 \\
\hline 448 & cytoplasmic & 0.083676 \\
\hline 449 & chaperone & 0.083508 \\
\hline 450 & fatty & 0.083409 \\
\hline 451 & class & 0.08311 \\
\hline 452 & $y f c z$ & 0.082864 \\
\hline 453 & $50 \mathrm{~S}$ & 0.082544 \\
\hline 454 & acids & 0.082296 \\
\hline 455 & bifunctional & 0.082202 \\
\hline 456 & CmeB & 0.08195 \\
\hline 457 & Nupc & 0.08195 \\
\hline 458 & NarP & 0.08195 \\
\hline 459 & BpeB & 0.08195 \\
\hline 460 & YpfH & 0.08195 \\
\hline 461 & ArpB & 0.08195 \\
\hline 462 & detox & 0.08195 \\
\hline 463 & citrate & 0.081808 \\
\hline 464 & purines & 0.081643 \\
\hline 465 & NarQ & 0.081631 \\
\hline 466 & to & 0.081556 \\
\hline 467 & integrase & 0.081461 \\
\hline 468 & zinc & 0.081406 \\
\hline 469 & UrtB & 0.08124 \\
\hline 470 & isochorismate & 0.081229 \\
\hline 471 & possible & 0.081212 \\
\hline 472 & cyanate & 0.081033 \\
\hline 473 & metabolite & 0.080956 \\
\hline 474 & switch & 0.080928 \\
\hline 475 & rhodanese & 0.080512 \\
\hline 476 & trans & 0.080495 \\
\hline 477 & xylose & 0.080484 \\
\hline 478 & endonuclease & 0.080381 \\
\hline 479 & S18 & 0.080338 \\
\hline 480 & uracil & 0.080215 \\
\hline 481 & diacetylchitobiose & 0.080184 \\
\hline 482 & cluster & 0.079937 \\
\hline 483 & leucine & 0.079413 \\
\hline 484 & PLP & 0.079397 \\
\hline
\end{tabular}




\begin{tabular}{|c|c|}
\hline 485 & homoserine \\
\hline 486 & nickel \\
\hline 487 & $\mathrm{NirC}$ \\
\hline 488 & ferrous \\
\hline 489 & $\operatorname{MalT}$ \\
\hline 490 & FGGY \\
\hline 491 & metal \\
\hline 492 & flavorubredoxin \\
\hline 493 & EmrB \\
\hline 494 & QacA \\
\hline 495 & $\operatorname{DctA}$ \\
\hline 496 & oxide \\
\hline 497 & acyltransferase \\
\hline 498 & 28 \\
\hline 499 & Probable \\
\hline 500 & UvrABC \\
\hline 501 & Gm22 51 \\
\hline 502 & gulonate \\
\hline 503 & VacB \\
\hline 504 & YjeB \\
\hline 505 & $\mathrm{H} f \mathrm{lB}$ \\
\hline 506 & $\mathrm{HF}$ \\
\hline 507 & DsbD \\
\hline 508 & Maltose \\
\hline 509 & polysaccharide \\
\hline 510 & FlgD \\
\hline 511 & topological \\
\hline 512 & Mo \\
\hline 513 & FlgG \\
\hline 514 & Gsic \\
\hline 515 & Der \\
\hline 516 & uridylate \\
\hline 517 & Twin \\
\hline 518 & Site \\
\hline 519 & ascorbate \\
\hline 520 & Pyrroline \\
\hline 521 & capping \\
\hline 522 & mulitfunctional \\
\hline 523 & SIS \\
\hline 524 & $\mathrm{HflK}$ \\
\hline 525 & or \\
\hline 526 & octulosonic \\
\hline 527 & repair \\
\hline 528 & cytochrome \\
\hline 529 & sensory \\
\hline 530 & exodeoxyribonuclease \\
\hline 531 & directed \\
\hline 532 & propionate \\
\hline 533 & erythritol \\
\hline 534 & flavin \\
\hline 535 & glycosylase \\
\hline 536 & YtfJ \\
\hline 537 & LepA \\
\hline 538 & Phop \\
\hline
\end{tabular}
0.079234
0.079227
0.079207
0.079207
0.079207
0.079128
0.07906
0.079045
0.078876
0.078876
0.078876
0.078696
0.078601
0.078467
0.078409
0.078317
0.078292
0.078292
0.078292
0.078292
0.078292
0.078292
0.078292
0.07817
0.077849
0.077752
0.077544
0.077544
0.077514
0.077378
0.077105
0.076849
0.076803
0.07623
0.07596
0.075922
0.075863
0.075812
0.075812
0.075812
0.075621
0.07533
0.075246
0.075197
0.075099
0.075085
0.075008
0.07411
0.07409
0.073914
0.073899
0.073872
0.073848
0.073848 


$\begin{array}{ll}539 & \text { Gals } \\ 540 & \text { RsuA } \\ 541 & \text { Rmf } \\ 542 & \text { YejH } \\ 543 & \text { YeiM } \\ 544 & \text { 516 } \\ 545 & \text { EIIBC } \\ 546 & \text { hemin } \\ 547 & \text { scaffolding } \\ 548 & \text { muramidase } \\ 549 & \text { NADP } \\ 550 & \text { GntR } \\ 551 & \text { nitrate } \\ 552 & \text { tryptophanyl } \\ 553 & \text { octaprenyl } \\ 554 & \text { excisionase } \\ 555 & \text { threonyl } \\ 556 & \text { branching } \\ 557 & \text { potasium } \\ 558 & \text { Uncharacterized } \\ 559 & \text { element } \\ 560 & \text { secretion } \\ 561 & \text { SufS } \\ 562 & \text { RpiR } \\ 563 & \text { pathway } \\ 564 & \text { regulon } \\ 565 & \text { cofactor } \\ 566 & \text { PAS } \\ 567 & \text { YcfS } \\ 568 & \text { YnhG } \\ 569 & \text { FliE } \\ 570 & \text { involved } \\ 571 & \text { diguanylate } \\ 572 & \text { division } \\ 573 & \text { serine } \\ 574 & \text { large } \\ 575 & \text { succinic } \\ 576 & \text { FixX } \\ 577 & \text { MucC } \\ 578 & \text { storage } \\ 579 & \text { cyano } \\ 580 & \text { deazaguanine } \\ 581 & \text { malic } \\ 582 & \text { length } \\ 583 & \text { deoR } \\ 584 & \text { neutral } \\ 585 & \text { an } \\ 586 & \text { FliM } \\ 587 & \text { fragment } \\ 588 & \text { EIIc } \\ 589 & \text { determining } \\ 590 & \text { subfamily } \\ 591 & \text { PAC } \\ 592 & \text { recombinases } \\ & \end{array}$
0.073721
0.073721
0.073721
0.073721
0.073721
0.073721
0.073721
0.073674
0.07362
0.073418
0.073354
0.073339
0.073244
0.072962
0.07276
0.072727
0.072622
0.072371
0.072351
0.072315
0.072158
0.072127
0.071932
0.071844
0.071614
0.07154
0.071207
0.070947
0.070945
0.070945
0.070829
0.070812
0.07075
0.070639
0.070584
0.070254
0.070079
0.070063
0.070063
0.06999
0.069729
0.069729
0.06971
0.06949
0.069475
0.069355
0.06927
0.068923
0.068858
0.068699
0.068645
0.068612
0.068542
0.068319 


\begin{tabular}{|c|c|}
\hline 593 & MFP \\
\hline 594 & utilization \\
\hline 595 & symporter \\
\hline 596 & ribonucleotide \\
\hline 597 & activity \\
\hline 598 & GltI \\
\hline 599 & monooxygenase \\
\hline 600 & HypD \\
\hline 601 & ring \\
\hline 602 & ribose \\
\hline 603 & NlpI \\
\hline 604 & flavodoxin \\
\hline 605 & sorbosone \\
\hline 606 & fagellar \\
\hline 607 & chloroplast \\
\hline 608 & ranscriptional \\
\hline 609 & Ybis \\
\hline 610 & ErfK \\
\hline 611 & 32 \\
\hline 612 & omega \\
\hline 613 & NrtA \\
\hline 614 & $4 \mathrm{Fe}$ \\
\hline 615 & $4 S$ \\
\hline 616 & lactose \\
\hline 617 & prolyl \\
\hline 618 & no \\
\hline 619 & $\mathrm{HAD}$ \\
\hline 620 & excinuclease \\
\hline 621 & histone \\
\hline 622 & modification \\
\hline 623 & proline \\
\hline 624 & FlgJ \\
\hline 625 & basal \\
\hline 626 & antitoxin \\
\hline 627 & maturation \\
\hline 628 & SPO1 \\
\hline 629 & disulfide \\
\hline 630 & body \\
\hline 631 & nucleic \\
\hline 632 & RseC \\
\hline 633 & $\mathrm{Zn}$ \\
\hline 634 & triphosphate \\
\hline 635 & anaerobic \\
\hline 636 & flis \\
\hline 637 & Spo0A \\
\hline 638 & Yqis \\
\hline 639 & aciddehydrogenase \\
\hline 640 & lipoamide \\
\hline 641 & Ptb \\
\hline 642 & uroporphyrinogen \\
\hline 643 & NasT \\
\hline 644 & PrkA \\
\hline 645 & RuBisCO \\
\hline 646 & mismatch \\
\hline
\end{tabular}
0.068213
0.068036
0.067973
0.067783
0.067675
0.067513
0.06747
0.067406
0.067389
0.067356
0.06732
0.067166
0.067126
0.066985
0.066941
0.06676
0.066716
0.066716
0.066618
0.066308
0.066072
0.065626
0.065498
0.06549
0.065485
0.065401
0.065349
0.065196
0.065159
0.065138
0.064852
0.064831
0.064801
0.064785
0.064678
0.064672
0.064558
0.064524
0.064354
0.064173
0.064088
0.064015
0.063787
0.063723
0.063662
0.063662
0.063662
0.063662
0.063662
0.063641
0.063445
0.063381
0.063289
0.063168 


\begin{tabular}{|c|c|}
\hline 647 & IF \\
\hline 648 & heptulosonate \\
\hline 649 & $\mathrm{SCOA}$ \\
\hline 650 & $Y x j C$ \\
\hline 651 & LL \\
\hline 652 & epimerase \\
\hline 653 & Non \\
\hline 654 & RutA \\
\hline 655 & sucrose \\
\hline 656 & CysD \\
\hline 657 & NÄGC \\
\hline 658 & specialized \\
\hline 659 & RpoX \\
\hline 660 & SpaP \\
\hline 661 & rge \\
\hline 662 & $\mathrm{XdhB}$ \\
\hline 663 & FtsH \\
\hline 664 & Thr \\
\hline 665 & cryptic \\
\hline 666 & facilitator \\
\hline 667 & $\mathrm{FlgC}$ \\
\hline 668 & rod \\
\hline 669 & degradation \\
\hline 670 & FlgM \\
\hline 671 & dihydroxyhept \\
\hline 672 & vitamin \\
\hline 673 & ANTAR \\
\hline 674 & NUDIX \\
\hline 675 & NO3 \\
\hline 676 & NO2 \\
\hline 677 & hook \\
\hline 678 & $\mathrm{Tu}$ \\
\hline 679 & repeat \\
\hline 680 & trehalose \\
\hline 681 & motor \\
\hline 682 & KefA \\
\hline 683 & WhiG \\
\hline 684 & urate \\
\hline 685 & FabA \\
\hline 686 & RtcB \\
\hline 687 & heptosyltransferase \\
\hline 688 & OMP 85 \\
\hline 689 & Gros \\
\hline 690 & GroEs3 \\
\hline 691 & HSP10 \\
\hline 692 & $r \mathrm{RNA}$ \\
\hline 693 & $\mathrm{C} 4$ \\
\hline 694 & spermidine \\
\hline 695 & flavoprotein \\
\hline 696 & acetolactate \\
\hline 697 & gluconate \\
\hline 698 & mutase \\
\hline 699 & induced \\
\hline 700 & pyridine \\
\hline
\end{tabular}

0.06313
0.062748
0.062748
0.062748
0.062748
0.062458
0.062303
0.062234
0.061957
0.061834
0.061834
0.061834
0.061834
0.061834
0.061834
0.061834
0.061782
0.061767
0.061705
0.061484
0.061475
0.061462
0.061424
0.061318
0.061229
0.061199
0.06108
0.060959
0.06094
0.06094
0.060921
0.060867
0.06083
0.060828
0.060643
0.060294
0.060294
0.060294
0.060294
0.060294
0.060097
0.060005
0.060005
0.060005
0.060005
0.060001
0.059983
0.059957
0.059704
0.059647
0.059472
0.059415
0.059355
0.059296 


$\begin{array}{ll}701 & \text { asparaginase } \\ 702 & \text { AzlC } \\ 703 & \text { anti } \\ 704 & \text { curli } \\ 705 & \text { Similar } \\ 706 & \text { amphiphile } \\ 707 & \text { HAE1 } \\ 708 & \text { LivH } \\ 709 & \text { L11 } \\ 710 & \text { dinitrogenase } \\ 711 & \text { flgf } \\ 712 & \text { Phe } \\ 713 & \text { aerobic } \\ 714 & \text { nonspecific } \\ 715 & \text { valine } \\ 716 & \text { Acetate } \\ 717 & \text { 16S } \\ 718 & \text { keto } \\ 719 & \text { damage } \\ 720 & \text { macrolide } \\ 721 & \text { solute } \\ 722 & \text { aminobutyrate } \\ 723 & \text { independent } \\ 724 & \text { amine } \\ 725 & \text { STP } \\ 726 & \text { SdaC } \\ 727 & \text { fuculose } \\ 728 & \text { Uvry } \\ 729 & \text { HofQ } \\ 730 & \text { NiFe } \\ 731 & \text { FeMo } \\ 732 & \text { glycerate } \\ 733 & \text { FAD } \\ 734 & \text { maltodextrin } \\ 735 & \text { stop } \\ 736 & \text { premature } \\ 737 & \text { FeS4 } \\ 738 & \text { LRV } \\ 739 & \text { alanine } \\ 740 & \text { Rof } \\ 741 & \text { YaeP } \\ 742 & \text { BfdA } \\ 743 & \text { ImpA } \\ 744 & \text { HcpA } \\ 745 & \text { Hcp } \\ 746 & \text { haemolysin } \\ 747 & \text { DnaG } \\ 748 & \text { 30S } \\ 749 & \text { dicarboxylate } \\ 750 & \text { stress } \\ 751 & \text { output } \\ 752 & \text { GAF } \\ 753 & \text { lysin } \\ 754 & \text { cyclohexanone } \\ & \end{array}$
0.059296
0.059213
0.059082
0.059065
0.059065
0.059001
0.059001
0.058946
0.058898
0.058756
0.058679
0.058537
0.058506
0.058354
0.05821
0.058138
0.058123
0.057957
0.057886
0.057842
0.057764
0.057747
0.0574
0.057379
0.057262
0.057262
0.057262
0.057262
0.057262
0.05706
0.056927
0.056895
0.056662
0.05647
0.056414
0.056414
0.056414
0.056414
0.056384
0.056347
0.056347
0.056347
0.056347
0.056347
0.056347
0.056347
0.056347
0.056302
0.056302
0.056186
0.056083
0.056059
0.056013
0.055996 


\begin{tabular}{|c|c|}
\hline 755 & aminoacylase \\
\hline 756 & pyrophosphokinase \\
\hline 757 & $\mathrm{Na}$ \\
\hline 758 & NUP \\
\hline 759 & respiratory \\
\hline 760 & allantoate \\
\hline 761 & toxin \\
\hline 762 & $\mathrm{TS}$ \\
\hline 763 & $F \lg B$ \\
\hline 764 & chemotaxis \\
\hline 765 & Rsu \\
\hline 766 & pseudouridine \\
\hline 767 & Di \\
\hline 768 & restriction \\
\hline 769 & hydroperoxide \\
\hline 770 & aldolase \\
\hline 771 & semialdehyde \\
\hline 772 & $F \lg E$ \\
\hline 773 & aldehyde \\
\hline 774 & F1F0 \\
\hline 775 & yngC \\
\hline 776 & YfjK \\
\hline 777 & $\mathrm{ACOA}$ \\
\hline 778 & PdhA \\
\hline 779 & HXTX \\
\hline 780 & GltP \\
\hline 781 & $\operatorname{NrfG}$ \\
\hline 782 & Mdto \\
\hline 783 & PhnJ \\
\hline 784 & $\operatorname{slyx}$ \\
\hline 785 & Csd \\
\hline 786 & CspA \\
\hline 787 & cspLB \\
\hline 788 & HpnJ \\
\hline 789 & $\mathrm{NADH}$ \\
\hline 790 & pantothenate \\
\hline 791 & lactate \\
\hline 792 & oxidase \\
\hline 793 & receiver \\
\hline 794 & stimulates \\
\hline 795 & fermentation \\
\hline 796 & alcohol \\
\hline 797 & aliphatic \\
\hline 798 & genes \\
\hline 799 & GcvR \\
\hline 800 & FHL \\
\hline 801 & $\mathrm{Bcp}$ \\
\hline 802 & m2G1207 \\
\hline 803 & HyfA \\
\hline 804 & dyp \\
\hline 805 & $933 \mathrm{R}$ \\
\hline 806 & packaging \\
\hline 807 & CheD \\
\hline 808 & NirD \\
\hline
\end{tabular}
0.055897
0.055815
0.055801
0.055768
0.055622
0.055613
0.055608
0.055555
0.055424
0.05539
0.055369
0.055311
0.055299
0.055193
0.055166
0.055037
0.054996
0.054958
0.05494
0.054519
0.054519
0.054519
0.054519
0.054519
0.054519
0.054519
0.054519
0.054519
0.054519
0.054519
0.054519
0.054519
0.054519
0.054519
0.054411
0.054046
0.054003
0.053941
0.053861
0.053786
0.053786
0.053713
0.053673
0.053629
0.053604
0.053604
0.053604
0.053604
0.053604
0.053604
0.053604
0.053604
0.053604
0.053604 


\begin{tabular}{|c|c|}
\hline 809 & mug \\
\hline 810 & recA \\
\hline 811 & NsrR \\
\hline 812 & carrier \\
\hline 813 & butyryltransferase \\
\hline 814 & ygfu \\
\hline 815 & ter \\
\hline 816 & TesB \\
\hline 817 & fuscose \\
\hline 818 & ybl121 \\
\hline 819 & GreA \\
\hline 820 & $\mathrm{EAT}$ \\
\hline 821 & acylglucosamine \\
\hline 822 & metalloprotease \\
\hline 823 & Hydantoin \\
\hline 824 & exoribonuclease \\
\hline 825 & urea \\
\hline 826 & attachment \\
\hline 827 & Ctc \\
\hline 828 & initiation \\
\hline 829 & Ser \\
\hline 830 & LacI \\
\hline 831 & malonyl \\
\hline 832 & aminoglycoside \\
\hline 833 & homogentisate \\
\hline 834 & putrescine \\
\hline 835 & auxiliary \\
\hline 836 & conductance \\
\hline 837 & Extracellular \\
\hline 838 & isozyme \\
\hline 839 & carbamoyltransferase \\
\hline 840 & thiolase \\
\hline 841 & NodT \\
\hline 842 & isoleucine \\
\hline 843 & adenosine \\
\hline 844 & surface \\
\hline 845 & bicyclomycin \\
\hline 846 & N6 \\
\hline 847 & CheY \\
\hline 848 & $\mathrm{ADP}$ \\
\hline 849 & cellobiose \\
\hline 850 & folding \\
\hline 851 & UreE \\
\hline 852 & Major \\
\hline 853 & AraC \\
\hline 854 & cystine \\
\hline 855 & PAAT \\
\hline 856 & Kdo \\
\hline 857 & WaaA \\
\hline 858 & $\operatorname{RfaG}$ \\
\hline 859 & RfaQ \\
\hline 860 & MscL \\
\hline 861 & m5C967 \\
\hline 862 & YCfR \\
\hline
\end{tabular}
0.053604
0.053269
0.053181
0.053091
0.052812
0.05269
0.05269
0.05269
0.05269
0.05269
0.052634
0.052535
0.052402
0.052344
0.052289
0.052202
0.051929
0.051897
0.051888
0.051822
0.05172
0.051644
0.051584
0.051545
0.051241
0.051117
0.050861
0.050839
0.050682
0.050618
0.05046
0.050448
0.050448
0.05031
0.050049
0.050019
0.049948
0.049948
0.049944
0.049916
0.049585
0.049562
0.049539
0.049532
0.04908
0.049034
0.049034
0.049032
0.049032
0.049032
0.049032
0.049032
0.049032
0.049032 


\begin{tabular}{|c|c|}
\hline 863 & longitudinal \\
\hline 864 & intracellular \\
\hline 865 & RadC \\
\hline 866 & PucI \\
\hline 867 & Folylpolyglutamate \\
\hline 868 & menaquinone \\
\hline 869 & group \\
\hline 870 & opeon \\
\hline 871 & $\mathrm{SC}$ \\
\hline 872 & svp \\
\hline 873 & $\mathrm{CCmB}$ \\
\hline 874 & CcmABCDE \\
\hline 875 & 140 \\
\hline 876 & Atod \\
\hline 877 & ato \\
\hline 878 & pahage \\
\hline 879 & YgjG \\
\hline 880 & QseC \\
\hline 881 & yecA \\
\hline 882 & Mred \\
\hline 883 & Cpn10 \\
\hline 884 & NasA \\
\hline 885 & nifr3 \\
\hline 886 & $\mathrm{XdhA}$ \\
\hline 887 & FlgK \\
\hline 888 & fumarate \\
\hline 889 & $\mathrm{HTH}$ \\
\hline 890 & NarL \\
\hline 891 & IMP \\
\hline 892 & short \\
\hline 893 & uptake \\
\hline 894 & Wza \\
\hline 895 & GsiB \\
\hline 896 & gsiABCD \\
\hline 897 & $\mathrm{Cmr}$ \\
\hline 898 & force \\
\hline 899 & motive \\
\hline 900 & $\operatorname{MdfA}$ \\
\hline 901 & $\mathrm{TPP}$ \\
\hline 902 & deaminase \\
\hline 903 & lysidine \\
\hline 904 & NifZ \\
\hline 905 & squalene \\
\hline 906 & hopene \\
\hline 907 & polyunsaturated \\
\hline 908 & PfaA \\
\hline 909 & MmuP \\
\hline 910 & IscAnif \\
\hline 911 & Low \\
\hline 912 & antibiotic \\
\hline 913 & CRP \\
\hline 914 & suppresses \\
\hline 915 & $\mathrm{HflX}$ \\
\hline 916 & $2 \mathrm{~S}$ \\
\hline
\end{tabular}
0.049032
0.048787
0.04867
0.048655
0.048655
0.0486
0.048406
0.048118
0.048118
0.048118
0.048118
0.048118
0.048118
0.048118
0.048118
0.048118
0.048118
0.048118
0.048118
0.048008
0.048008
0.048008
0.047978
0.047978
0.047933
0.04776
0.047728
0.047494
0.047494
0.047378
0.047213
0.047204
0.047204
0.047204
0.047204
0.047204
0.047204
0.047204
0.046922
0.046435
0.04641
0.046394
0.046289
0.046289
0.046289
0.046289
0.046289
0.046289
0.046244
0.046214
0.045921
0.04592
0.045866
0.04578 


\begin{tabular}{|c|c|}
\hline 917 & $2 \mathrm{Fe}$ \\
\hline 918 & $23 S$ \\
\hline 919 & $\mathrm{ACP}$ \\
\hline 920 & yddR \\
\hline 921 & deacetylase \\
\hline 922 & SugE \\
\hline 923 & quaternary \\
\hline 924 & Atos \\
\hline 925 & homocysteine \\
\hline 926 & Exonuclease \\
\hline 927 & Luciferase \\
\hline 928 & converting \\
\hline 929 & FtsE \\
\hline 930 & entericidin \\
\hline 931 & deoxyheptonate \\
\hline 932 & UPF0319 \\
\hline 933 & deoxyguanosinetriphosphate \\
\hline 934 & U8 \\
\hline 935 & $\mathrm{ACN}$ \\
\hline 936 & siderophore \\
\hline 937 & MS \\
\hline 938 & butyrate \\
\hline 939 & 60 \\
\hline 940 & sodium \\
\hline 941 & aminopeptidase \\
\hline 942 & Mal \\
\hline 943 & dipeptidyl \\
\hline 944 & biotin \\
\hline 945 & trancsriptional \\
\hline 946 & rubrerythrin \\
\hline 947 & $\mathrm{box}$ \\
\hline 948 & Glutaredoxin \\
\hline 949 & VII \\
\hline 950 & $\mathrm{HflC}$ \\
\hline 951 & Insulin \\
\hline 952 & $\mathrm{EF}$ \\
\hline 953 & delta \\
\hline 954 & SoxR \\
\hline 955 & subIIB \\
\hline 956 & $\operatorname{manL}$ \\
\hline 957 & hydratase \\
\hline 958 & FlhF \\
\hline 959 & aminohexanoate \\
\hline 960 & release \\
\hline 961 & pyrimidine \\
\hline 962 & sulfatase \\
\hline 963 & zeaxanthin \\
\hline 964 & $\mathrm{Aba} 2$ \\
\hline 965 & thiol \\
\hline 966 & undecaprenyl \\
\hline 967 & mutH \\
\hline 968 & $\operatorname{ICC}$ \\
\hline 969 & M2 8 \\
\hline 970 & Trk \\
\hline
\end{tabular}

0.04578

0.045744

0.045735

0.045422

0.045419

0.045338

0.045338

0.045338

0.045316

0.045111

0.045085

0.04507

0.04504

0.045038

0.04495

0.04481

0.044627

0.044461

0.044461

0.044365

0.044222

0.044124

0.044124

0.044078

0.043999

0.043965

0.043921

0.043693

0.043667

0.043657

0.043624

0.043465

0.043341

0.043226

0.043226

0.04301

0.042956

0.042698

0.042632

0.042632

0.042452

0.042295

0.042098

0.042058

0.041727

0.041721

0.041717

0.041717

0.041712

0.041646

0.041543

0.041543

0.041543

0.04138 


\begin{tabular}{|c|c|c|}
\hline 971 & bicarbonate & 0.041196 \\
\hline 972 & aromatic & 0.041196 \\
\hline 973 & compound & 0.041149 \\
\hline 974 & M50 & 0.041114 \\
\hline 975 & IV & 0.041091 \\
\hline 976 & aldo & 0.041036 \\
\hline 977 & hypoxanthine & 0.04095 \\
\hline 978 & AtoC & 0.040896 \\
\hline 979 & universal & 0.040859 \\
\hline 980 & xylulokinase & 0.040802 \\
\hline 981 & diphosphate & 0.040773 \\
\hline 982 & Casl & 0.040586 \\
\hline 983 & porter & 0.040586 \\
\hline 984 & acetoacetyl & 0.040511 \\
\hline 985 & DctM & 0.040466 \\
\hline 986 & CspD & 0.040249 \\
\hline 987 & $6 \mathrm{x}$ & 0.040249 \\
\hline 988 & FxsA & 0.040249 \\
\hline 989 & gene & 0.040231 \\
\hline 990 & FliR & 0.04023 \\
\hline 991 & selenium & 0.04023 \\
\hline 992 & including & 0.040099 \\
\hline 993 & FtsW & 0.040058 \\
\hline 994 & $\mathrm{PEP}$ & 0.04001 \\
\hline 995 & SohA & 0.039889 \\
\hline 996 & YhaV & 0.039889 \\
\hline 997 & TupA & 0.039889 \\
\hline 998 & MFS & 0.039854 \\
\hline 999 & Electron & 0.039808 \\
\hline 1000 & inhibitor & 0.039804 \\
\hline 1001 & UPF0005 & 0.039603 \\
\hline 1002 & $\operatorname{RecB}$ & 0.039603 \\
\hline 1003 & $\mathrm{Ffh}$ & 0.039551 \\
\hline 1004 & $\mathrm{BCr}$ & 0.03953 \\
\hline 1005 & UvrB & 0.03953 \\
\hline 1006 & NCS1 & 0.03953 \\
\hline 1007 & encoded & 0.03953 \\
\hline 1008 & drug & 0.039526 \\
\hline 1009 & positive & 0.039132 \\
\hline 1010 & dioxygenase & 0.039109 \\
\hline 1011 & ribulose & 0.039094 \\
\hline 1012 & ketoacyl & 0.039063 \\
\hline 1013 & $\mathrm{HI}$ & 0.038956 \\
\hline 1014 & SecE & 0.038905 \\
\hline 1015 & alkaline & 0.038637 \\
\hline 1016 & channel & 0.038455 \\
\hline 1017 & recognition & 0.038425 \\
\hline 1018 & FlaA & 0.03831 \\
\hline 1019 & IS2 & 0.03831 \\
\hline 1020 & fucose & 0.03831 \\
\hline 1021 & RecBCD & 0.03831 \\
\hline 1022 & Chew & 0.038232 \\
\hline 1023 & hydrocarbon & 0.03818 \\
\hline 1024 & prophage & 0.038167 \\
\hline
\end{tabular}




$\begin{array}{ll}1025 & \text { NusG } \\ 1026 & \text { MutT } \\ 1027 & \text { Flix } \\ 1028 & \text { mgtE } \\ 1029 & \text { LemA } \\ 1030 & \text { sequence } \\ 1031 & \text { urease } \\ 1032 & \text { cobalamin } \\ 1033 & \text { vanX } \\ 1034 & \text { M15D } \\ 1035 & \text { Yaed } \\ 1036 & \text { proton } \\ 1037 & \text { oxo } \\ 1038 & \text { alkylhydroperoxidase } \\ 1039 & \text { Grot } \\ 1040 & \text { RocR } \\ 1041 & \text { Control } \\ 1042 & \text { import } \\ 1043 & \text { exchanger } \\ 1044 & \text { CheA } \\ 1045 & \text { sulfoxide } \\ 1046 & \text { GbdR } \\ 1047 & \text { GlxA } \\ 1048 & \text { NRZ } \\ 1049 & \text { DdpA } \\ 1050 & \text { DdpX } \\ 1051 & \text { KgtP } \\ 1052 & \text { FixB } \\ 1053 & \text { DctD } \\ 1054 & \text { PcaT } \\ 1055 & \text { flgI } \\ 1056 & \text { L1 } \\ 1057 & \text { alanyl } \\ 1058 & \text { tyrosine } \\ 1059 & \text { Co } \\ 1060 & \text { catalytic } \\ 1061 & \text { pump } \\ 1062 & \text { lipoyl } \\ 1063 & \text { FkbM } \\ 1064 & \text { Sel1 } \\ 1065 & \text { EIIAB } \\ 1066 & \text { receptor } \\ 1067 & \text { oxalurate } \\ 1068 & \text { Ggt } \\ 1069 & \text { ywrD } \\ 1070 & \text { Heat } \\ 1071 & \text { Hfq } \\ 1072 & \text { L7 } \\ 1073 & \text { L12 } \\ 1074 & \text { adenine } \\ 1075 & \text { exopolysaccharide } \\ 1076 & \text { core } \\ 1077 & \text { genetic } \\ 1078 & \text { tetrahydropyridine } \\ & \\ 1001\end{array}$
0.038087
0.038087
0.03806
0.03806
0.037946
0.037888
0.037868
0.037672
0.037663
0.037663
0.037663
0.03756
0.037464
0.037464
0.037418
0.037418
0.037394
0.037388
0.037369
0.037265
0.037201
0.037146
0.037146
0.037146
0.037146
0.037146
0.037146
0.037146
0.037146
0.037146
0.037016
0.036773
0.036768
0.03665
0.036577
0.036576
0.036574
0.036427
0.03637
0.03637
0.036362
0.036324
0.036231
0.036231
0.036231
0.036043
0.036042
0.035893
0.035893
0.03587
0.03587
0.035864
0.035835
0.035775 


\begin{tabular}{|c|c|}
\hline 1079 & NifW \\
\hline 1080 & NNP \\
\hline 1081 & CysB \\
\hline 1082 & LambdaBa 04 \\
\hline 1083 & GCVA \\
\hline 1084 & deoxyphosphooctonate \\
\hline 1085 & lysyl \\
\hline 1086 & TonB \\
\hline 1087 & Rossmann \\
\hline 1088 & usher \\
\hline 1089 & PtsN \\
\hline 1090 & septal \\
\hline 1091 & MqsRA \\
\hline 1092 & MqsR \\
\hline 1093 & MqsA \\
\hline 1094 & that \\
\hline 1095 & divisome \\
\hline 1096 & ribosyltransferase \\
\hline 1097 & amidohydrolase \\
\hline 1098 & bacterial \\
\hline 1099 & redoxin \\
\hline 1100 & shock \\
\hline 1101 & nark \\
\hline 1102 & RNase \\
\hline 1103 & palmitoleoyl \\
\hline 1104 & heme \\
\hline 1105 & central \\
\hline 1106 & dephospho \\
\hline 1107 & Internalin \\
\hline 1108 & ceramidase \\
\hline 1109 & from \\
\hline 1110 & FlgN \\
\hline 1111 & DUF2 126 \\
\hline 1112 & Fnr \\
\hline 1113 & TIM \\
\hline 1114 & CoxL \\
\hline 1115 & CutL \\
\hline 1116 & protecting \\
\hline 1117 & acting \\
\hline 1118 & mechanosensitive \\
\hline 1119 & triosephosphate \\
\hline 1120 & sulfonate \\
\hline 1121 & general \\
\hline 1122 & galactosidase \\
\hline 1123 & IscA \\
\hline 1124 & $F \lg A$ \\
\hline 1125 & DSBA \\
\hline 1126 & GstI \\
\hline 1127 & strand \\
\hline 1128 & oxoisovalerate \\
\hline 1129 & presentation \\
\hline 1130 & chemoreceptor \\
\hline 1131 & Cystathionine \\
\hline 1132 & rotamase \\
\hline
\end{tabular}

0.035723

0.035723

0.035723

0.035723

0.035723

0.035633

0.035546

0.03552

0.035436

0.035436

0.035436

0.035317

0.035317

0.035317

0.035317

0.035317

0.035317

0.03527

0.035172

0.035124

0.035044

0.035008

0.034979

0.034815

0.034779

0.034686

0.03463

0.034601

0.034443

0.03443

0.034406

0.034406

0.034402

0.034287

0.034257

0.034251

0.034251

0.034251

0.034251

0.034157

0.034064

0.033942

0.033827

0.033807

0.033783

0.033683

0.033607

0.033488

0.033338

0.033195

0.033195

0.033137

0.032764

0.032667 


\begin{tabular}{|c|c|}
\hline 1133 & PAAR \\
\hline 1134 & ferrodoxin \\
\hline 1135 & CvrA \\
\hline 1136 & NhaP \\
\hline 1137 & volume \\
\hline 1138 & CDP \\
\hline 1139 & host \\
\hline 1140 & Man \\
\hline 1141 & aerotaxis \\
\hline 1142 & three \\
\hline 1143 & rhs \\
\hline 1144 & L33 \\
\hline 1145 & S2 \\
\hline 1146 & IS5 \\
\hline 1147 & E1 \\
\hline 1148 & light \\
\hline 1149 & Ala \\
\hline 1150 & GMP \\
\hline 1151 & oxoadipate \\
\hline 1152 & esterase \\
\hline 1153 & nicotinamidase \\
\hline 1154 & replication \\
\hline 1155 & $\mathrm{CcmI}$ \\
\hline 1156 & FleR \\
\hline 1157 & FleQ \\
\hline 1158 & ISCrol \\
\hline 1159 & ptxB \\
\hline 1160 & sector \\
\hline 1161 & SA0 881 \\
\hline 1162 & Adh3 \\
\hline 1163 & apoprotein \\
\hline 1164 & ClpB \\
\hline 1165 & rho \\
\hline 1166 & sporulation \\
\hline 1167 & antigen \\
\hline 1168 & thiosulfate \\
\hline 1169 & LPS \\
\hline 1170 & SDR \\
\hline 1171 & ferric \\
\hline 1172 & chloramphenicol \\
\hline 1173 & bisphosphatase \\
\hline 1174 & diacylglyceryl \\
\hline 1175 & Hsp90 \\
\hline 1176 & Hemolysin \\
\hline 1177 & thermostable \\
\hline 1178 & lauroyl \\
\hline 1179 & glutathione \\
\hline 1180 & guanylate \\
\hline 1181 & Chitooligosaccharide \\
\hline 1182 & uricase \\
\hline 1183 & ureidoglycolate \\
\hline 1184 & Yen8 \\
\hline 1185 & avirulence \\
\hline 1186 & DsdX \\
\hline
\end{tabular}

0.032667

0.032667

0.032574

0.032574

0.032574

0.032512

0.03251

0.03249

0.03249

0.03249

0.032235

0.032235

0.032205

0.032139

0.031954

0.031952

0.031933

0.031891

0.031844

0.031765

0.031759

0.031692

0.031659

0.031659

0.031659

0.031659

0.031659

0.031659

0.031659

0.031659

0.031659

0.031659

0.031587

0.031558

0.031464

0.031367

0.031367

0.031281

0.031204

0.031197

0.031113

0.030991

0.030896

0.030881

0.030863

0.030863

0.030838

0.0308

0.030745

0.030745

0.030745

0.030745

0.030745

0.030745 


$\begin{array}{ll}1187 & \text { acriflavin } \\ 1188 & \text { FlaG } \\ 1189 & \text { Hcpl } \\ 1190 & \text { hopanoid } \\ 1191 & \text { polA } \\ 1192 & \text { YfhF } \\ 1193 & \text { YadR } \\ 1194 & \text { HesB } \\ 1195 & \text { IscN } \\ 1196 & \text { inositol } \\ 1197 & \text { oxoglutarate } \\ 1198 & \text { region } \\ 1199 & \text { methoxyphenyl } \\ 1200 & \text { plastoquinone } \\ 1201 & \text { filamentation } \\ 1202 & \text { preprotein } \\ 1203 & \text { dichlorophenolindophenol } \\ 1204 & \text { MoFe } \\ 1205 & \text { imidazole } \\ 1206 & \text { GabT } \\ 1207 & \text { IF3 } \\ 1208 & \text { PolX } \\ 1209 & \text { GDH } \\ 1210 & \text { RocG3 } \\ 1211 & \text { RocG } \\ 1212 & \text { MtnE } \\ 1213 & \text { MsuE } \\ 1214 & \text { MsuD } \\ 1215 & \text { L21 } \\ 1216 & \text { isopropylmalate } \\ 1217 & \text { quinolinate } \\ 1218 & \text { cytosine } \\ 1219 & \text { L3 } \\ 1220 & \text { apc } \\ 1221 & \text { hydro } \\ 1222 & \text { AsnC } \\ 1223 & \text { sorbose } \\ 1224 & \text { finger } \\ 1225 & \text { biopolymer } \\ 1226 & \text { oxidative } \\ 1227 & \text { desuccinylase } \\ 1228 & \text { phage } \\ 1229 & \text { Gsid } \\ 1230 & \text { YqeF } \\ 1231 & \text { thil } \\ 1232 & \text { ThlA } \\ 1233 & \text { KamA } \\ 1234 & \text { CodB } \\ 1235 & \text { Yodo } \\ 1236 & \text { dinuclear } \\ 1237 & \text { adhesion } \\ 1238 & \text { Vgr } \\ 1239 & \text { Lrp } \\ 1240 & \text { TerC } \\ & \end{array}$
0.030734
0.030725
0.030555
0.030555
0.03055
0.03055
0.03055
0.03055
0.03055
0.030543
0.030504
0.030479
0.030406
0.030406
0.030397
0.030183
0.030027
0.029904
0.029877
0.029831
0.029831
0.029831
0.029831
0.029831
0.029831
0.029831
0.029831
0.029831
0.029776
0.029775
0.029719
0.0295
0.029499
0.029499
0.029492
0.029489
0.029478
0.029156
0.029034
0.029029
0.029029
0.028974
0.028916
0.028916
0.028916
0.028916
0.028916
0.028916
0.028916
0.02865
0.02865
0.028577
0.028476
0.028476 


$\begin{array}{ll}1241 & \text { FliW } \\ 1242 & \text { Tpx } \\ 1243 & \text { tungstate } \\ 1244 & \text { on } \\ 1245 & \text { CcdB } \\ 1246 & \text { YgeD } \\ 1247 & \text { Escherichia } \\ 1248 & \text { HoxS } \\ 1249 & \text { HupV } \\ 1250 & \text { derivatives } \\ 1251 & \text { GuaD } \\ 1252 & \text { fec } \\ 1253 & \text { IS629 } \\ 1254 & \text { epoxide } \\ 1255 & \text { glucarate } \\ 1256 & \text { particle } \\ 1257 & \text { FlhA } \\ 1258 & \text { RocE } \\ 1259 & \text { RocD } \\ 1260 & \text { RapG } \\ 1261 & \text { LuxR } \\ 1262 & \text { colicin } \\ 1263 & \text { IS } \\ 1264 & \text { PE } \\ 1265 & \text { oxygenase } \\ 1266 & \text { SecF } \\ 1267 & \text { maf } \\ 1268 & \text { AAA } \\ 1269 & \text { IclR } \\ 1270 & \text { M14 } \\ 1271 & \text { L25 } \\ 1272 & \text { MmsB } \\ 1273 & \text { hibadh } \\ 1274 & \text { PstA } \\ 1275 & \text { linoleoyl } \\ 1276 & \text { Lat } \\ 1277 & \text { PmmA } \\ 1278 & \text { YgjR } \\ 1279 & \text { yqjE } \\ 1280 & \text { alx } \\ 1281 & \text { dieonyl } \\ 1282 & \text { during } \\ 1283 & \text { SUHB } \\ 1284 & \text { extragenic } \\ 1285 & \text { BFD } \\ 1286 & \text { Capsid } \\ 1287 & \text { related } \\ 1288 & \text { formyltransferase } \\ 1289 & \text { F0 } \\ 1290 & \text { kD } \\ 1291 & \text { DctQ } \\ 1292 & \text { SMR } \\ 1293 & \text { ClpA } \\ 1294 & \text { FtsI } \\ & \\ 1250\end{array}$
0.028443
0.028443
0.028271
0.02812
0.028002
0.028002
0.028002
0.028002
0.028002
0.028002
0.028002
0.028002
0.027964
0.027862
0.027663
0.027487
0.027271
0.027087
0.027087
0.027087
0.027063
0.027044
0.027044
0.026789
0.026674
0.026671
0.026671
0.026562
0.026548
0.026331
0.026193
0.026173
0.026173
0.026173
0.026173
0.026173
0.026173
0.026173
0.026173
0.026173
0.026173
0.026173
0.026024
0.026024
0.026024
0.026024
0.025889
0.025877
0.025834
0.025834
0.025803
0.025803
0.025803
0.025803 


\begin{tabular}{|c|c|}
\hline 1295 & amidotransferase \\
\hline 1296 & epsilon \\
\hline 1297 & inducible \\
\hline 1298 & antiporter \\
\hline 1299 & Dps \\
\hline 1300 & CRISPR \\
\hline 1301 & distal \\
\hline 1302 & Saes \\
\hline 1303 & fibrinogen \\
\hline 1304 & $R n f G$ \\
\hline 1305 & Blr \\
\hline 1306 & yico \\
\hline 1307 & YieG \\
\hline 1308 & YpdF \\
\hline 1309 & AzgA \\
\hline 1310 & $\mathrm{AD}$ \\
\hline 1311 & $\mathrm{RPME}$ \\
\hline 1312 & MadM \\
\hline 1313 & YufO \\
\hline 1314 & ase \\
\hline 1315 & aspQ \\
\hline 1316 & AnsB \\
\hline 1317 & L31P \\
\hline 1318 & tRNAGln \\
\hline 1319 & MdtP \\
\hline 1320 & shikimate \\
\hline 1321 & Acetoin \\
\hline 1322 & YggA \\
\hline 1323 & lactamase \\
\hline 1324 & solvent \\
\hline 1325 & dehalogenase \\
\hline 1326 & salicylate \\
\hline 1327 & MHS \\
\hline 1328 & monoxide \\
\hline 1329 & $\mathrm{CofH}$ \\
\hline 1330 & $n d k A$ \\
\hline 1331 & NDK \\
\hline 1332 & fbic \\
\hline 1333 & RPST \\
\hline 1334 & EIS \\
\hline 1335 & apyrimidinic \\
\hline 1336 & didemethyl \\
\hline 1337 & deazariboflavin \\
\hline 1338 & $\operatorname{DadA}$ \\
\hline 1339 & UreF \\
\hline 1340 & UxuR \\
\hline 1341 & polyhydroxyalkanoate \\
\hline 1342 & RES \\
\hline 1343 & oxoacid \\
\hline 1344 & ppGpp \\
\hline 1345 & Protoheme \\
\hline 1346 & maleylacetoacetate \\
\hline 1347 & plug \\
\hline 1348 & Rne \\
\hline
\end{tabular}
0.025725
0.025546
0.025482
0.025372
0.025364
0.025295
0.025275
0.025259
0.025259
0.025259
0.025259
0.025259
0.025259
0.025259
0.025259
0.025259
0.025259
0.025259
0.025259
0.025259
0.025259
0.025259
0.025259
0.025259
0.025259
0.025151
0.025019
0.025019
0.025012
0.024999
0.024788
0.024747
0.024731
0.024548
0.024344
0.024344
0.024344
0.024344
0.024344
0.024344
0.024344
0.024344
0.024344
0.024344
0.024344
0.024344
0.024344
0.024327
0.024327
0.024219
0.024219
0.024219
0.024219
0.024219 


$\begin{array}{ll}1349 & \text { GTPase } \\ 1350 & \text { precorrin } \\ 1351 & \text { Strs } \\ 1352 & \text { EryC1 } \\ 1353 & \text { DnrJ } \\ 1354 & \text { DegT } \\ 1355 & \text { FixA } \\ 1356 & \text { Polyphosphate } \\ 1357 & \text { tautomerase } \\ 1358 & \text { GCN5 } \\ 1359 & \text { CAMP } \\ 1360 & \text { Coenzyme } \\ 1361 & \text { kDa } \\ 1362 & \text { RubA } \\ 1363 & \text { FprB } \\ 1364 & \text { AR } \\ 1365 & \text { adrenodoxin } \\ 1366 & \text { LysS } \\ 1367 & \text { bacilysin } \\ 1368 & \text { YwfH } \\ 1369 & \text { LivG } \\ 1370 & \text { FdhD } \\ 1371 & \text { oxoacyl } \\ 1372 & \text { long } \\ 1373 & \text { Orf2 } \\ 1374 & \text { ketoglutarate } \\ 1375 & \text { 10 } \\ 1376 & \text { IS1 } \\ 1377 & \text { carbon } \\ 1378 & \text { valyl } \\ 1379 & \text { helix } \\ 1380 & \text { RpoN } \\ 1381 & \text { anchored } \\ 1382 & \text { FliP } \\ 1383 & \text { GPM2 } \\ 1384 & \text { SOJ } \\ 1385 & \text { NiCoT } \\ 1386 & \text { NICT } \\ 1387 & \text { Ni2 } \\ 1388 & \text { NixA } \\ 1389 & \text { BPG } \\ 1390 & \text { Mtr } \\ 1391 & \text { HoxN } \\ 1392 & \text { HupN } \\ 1393 & \text { transition } \\ 1394 & \text { PGAM } \\ 1395 & \text { PhaC1 } \\ 1396 & \text { PhaA } \\ 1397 & \text { PHA } \\ 1398 & \text { GlgP } \\ 1399 & \text { LppF } \\ 1400 & \text { NRII } \\ 1401 & \text { LysE } \\ 1402 & \text { primase } \\ 1 & \end{array}$
0.02421
0.024181
0.024084
0.024084
0.024084
0.024084
0.024084
0.024081
0.023929
0.023692
0.023547
0.023542
0.023441
0.023438
0.02343
0.02343
0.02343
0.02343
0.02343
0.02343
0.02343
0.023428
0.023374
0.023367
0.023363
0.023362
0.023282
0.02309
0.022981
0.022944
0.022624
0.022582
0.022553
0.022545
0.022516
0.022516
0.022516
0.022516
0.022516
0.022516
0.022516
0.022516
0.022516
0.022516
0.022516
0.022516
0.022516
0.022516
0.022516
0.022516
0.022516
0.022516
0.022312
0.022312 


$\begin{array}{ll}1403 & \text { F0F1 } \\ 1404 & \text { glycoprotease } \\ 1405 & 70 \\ 1406 & \text { Flik } \\ 1407 & \text { PGRS } \\ 1408 & \text { YdiF } \\ 1409 & \text { paraquat } \\ 1410 & \text { Obge } \\ 1411 & \text { tail } \\ 1412 & \text { oligopeptide } \\ 1413 & \text { Fe3 } \\ 1414 & \text { lppQ } \\ 1415 & \text { CysC } \\ 1416 & \text { CysN } \\ 1417 & \text { NrgA } \\ 1418 & \text { BraG } \\ 1419 & \text { monohaem } \\ 1420 & \text { LivF } \\ 1421 & \text { drugs } \\ 1422 & \text { WbbL1 } \\ 1423 & \text { CpsA } \\ 1424 & \text { NH3 } \\ 1425 & \text { RmlD } \\ 1426 & \text { rha } \\ 1427 & \text { YohC } \\ 1428 & 558 \\ 1429 & \text { ARSC } \\ 1430 & \text { lyxo } \\ 1431 & \text { PVDS } \\ 1432 & \text { UvrBC } \\ 1433 & \text { uvra1 } \\ 1434 & \text { cbb3 } \\ 1435 & \text { prolipoprotein } \\ 1436 & \text { ArgR } \\ 1437 & \text { V5 } \\ 1438 & \text { GroES } \\ 1439 & \text { radical } \\ 1440 & \text { VI } \\ 1441 & \text { deadenyltransferase } \\ 1442 & \text { hrcA } \\ 1443 & \text { FlgH } \\ 1444 & \text { ORF1 } \\ 1445 & \text { PrlF } \\ 1446 & \text { sialic } \\ 1447 & \text { IIAB } \\ 1448 & \text { sedoheptulose } \\ 1449 & \text { CtpG } \\ 1450 & \text { MdtH } \\ 1451 & \text { YceB } \\ 1452 & \text { ybbP } \\ 1453 & \text { HDDA } \\ 1454 & \text { primary } \\ 1455 & \text { SsuD1 } \\ 1456 & \text { fmnh } \\ & \end{array}$

0.022312

0.022308

0.022306

0.022188

0.022175

0.022145

0.021989

0.021935

0.021837

0.021808

0.021718

0.021601

0.021601

0.021601

0.021601

0.021601

0.021601

0.021601

0.021601

0.021601

0.021601

0.021601

0.021601

0.021601

0.021601

0.021601

0.021601

0.021601

0.021601

0.021601

0.021601

0.021579

0.021561

0.021498

0.021498

0.021478

0.021443

0.021349

0.021261

0.021261

0.021261

0.021188

0.021051

0.020851

0.020814

0.020687

0.020687

0.020687

0.020687

0.020687

0.020687

0.020687

0.020687

0.020687 


$\begin{array}{ll}1457 & \text { L21P } \\ 1458 & \text { MobA } \\ 1459 & \text { HtrA } \\ 1460 & \text { FKBP } \\ 1461 & \text { CBS } \\ 1462 & \text { selenocysteine } \\ 1463 & \text { biogenesis } \\ 1464 & \text { sn } \\ 1465 & \text { TorD } \\ 1466 & \text { NifX } \\ 1467 & \text { FleS } \\ 1468 & \text { redox } \\ 1469 & \text { S8 } \\ 1470 & \text { heptaprenyl } \\ 1471 & \text { ribityllumazine } \\ 1472 & \text { NDP } \\ 1473 & \text { supressor } \\ 1474 & \text { CbpM } \\ 1475 & \text { CynS } \\ 1476 & \text { amyloplastic } \\ 1477 & \text { addition } \\ 1478 & \text { LfgB } \\ 1479 & \text { laccase } \\ 1480 & \text { ZMP1 } \\ 1481 & \text { rnfH } \\ 1482 & \text { M13 } \\ 1483 & \text { ASASE } \\ 1484 & \text { ASL } \\ 1485 & \text { PURB } \\ 1486 & \text { NRAMP } \\ 1487 & \text { GlcNAC } \\ 1488 & \text { Fe4S4 } \\ 1489 & \text { AMP } \\ 1490 & \text { nuclease } \\ 1491 & \text { penicillin } \\ 1492 & \text { NmrA } \\ 1493 & \text { hybrid } \\ 1494 & \text { deformylase } \\ 1495 & \text { enoyl } \\ 1496 & \text { Cold } \\ 1497 & \text { extrusion } \\ 1498 & \text { SecG } \\ 1499 & \text { altronate } \\ 1500 & \text { SRP54 } \\ 1501 & \text { orotidine } \\ 1502 & \text { EutR } \\ 1503 & \text { NusB } \\ 1504 & \text { MCP } \\ 1505 & \text { dTDP } \\ 1506 & \text { barrel } \\ 1507 & \text { coaE } \\ 1508 & \text { trpC } \\ 1509 & \text { yohN } \\ 1510 & \text { senter } \\ & \end{array}$
0.020687
0.020523
0.020523
0.020441
0.020441
0.02033
0.020252
0.020205
0.020205
0.020205
0.020091
0.020091
0.020068
0.019995
0.019889
0.019889
0.019772
0.019772
0.019772
0.019772
0.019772
0.019772
0.019772
0.019772
0.019772
0.019772
0.019772
0.019772
0.019772
0.019682
0.019682
0.019682
0.019613
0.019459
0.019433
0.019432
0.019427
0.019337
0.019337
0.019145
0.019142
0.018975
0.018975
0.018939
0.018939
0.018939
0.018912
0.018912
0.018873
0.018868
0.018858
0.018858
0.018858
0.018858 


$\begin{array}{ll}1511 & \text { CTPB } \\ 1512 & \text { yagt } \\ 1513 & \text { UPF0231 } \\ 1514 & \text { AscG } \\ 1515 & \text { HycI } \\ 1516 & \text { HydN } \\ 1517 & \text { deoxyoctulosonic } \\ 1518 & \text { AGzA } \\ 1519 & \text { phytochrome } \\ 1520 & \text { arabinose } \\ 1521 & \text { sarcosine } \\ 1522 & \text { part } \\ 1523 & \text { SecD } \\ 1524 & \text { depolymerase } \\ 1525 & \text { fhuC } \\ 1526 & \text { RecX } \\ 1527 & \text { inactive } \\ 1528 & \text { FliF } \\ 1529 & \text { Hpt } \\ 1530 & \text { HydA } \\ 1531 & \text { Chorismate } \\ 1532 & \text { cyanophycin } \\ 1533 & \text { fusaric } \\ 1534 & \text { UgpC } \\ 1535 & \text { dnaC } \\ 1536 & \text { DnaI } \\ 1537 & \text { OruR } \\ 1538 & \text { OppD } \\ 1539 & \text { NorA } \\ 1540 & \text { RCMNS } \\ 1541 & \text { YesN } \\ 1542 & \text { MenB } \\ 1543 & \text { lipid } \\ 1544 & \text { Chitin } \\ 1545 & \text { multicopper } \\ 1546 & \text { osmotically } \\ 1547 & \text { Hrp } \\ 1548 & \text { malonate } \\ 1549 & \text { Hup } \\ 1550 & \text { triacylglycerol } \\ 1551 & \text { cobalt } \\ 1552 & \text { dimethylallyl } \\ 1553 & \text { tartrate } \\ 1554 & \text { UreD } \\ 1555 & \text { oxononanoate } \\ 1556 & \text { TRAP } \\ 1557 & \text { mannose } \\ 1558 & \text { Polynucleotide } \\ 1559 & \text { L9 } \\ 1560 & \text { butanediol } \\ 1562 & \text { SHPD } \\ 1563 & \text { Chez } \\ 1564 & \text { amaB } \\ & \end{array}$
0.018858
0.018858
0.018858
0.018858
0.018858
0.018858
0.018858
0.018858
0.018858
0.018715
0.018584
0.018574
0.018455
0.018455
0.018265
0.018265
0.018265
0.018104
0.01806
0.01806
0.018006
0.017944
0.017944
0.017944
0.017944
0.017944
0.017944
0.017944
0.017944
0.017944
0.017944
0.017944
0.017872
0.017827
0.017759
0.017637
0.017619
0.017619
0.017619
0.017603
0.017543
0.017458
0.017454
0.017413
0.017413
0.017279
0.017235
0.017146
0.017146
0.017146
0.017081
0.017029
0.017029
0.017029 


$\begin{array}{ll}1565 & \text { FljK } \\ 1566 & \text { FljM } \\ 1567 & \text { FljL } \\ 1568 & \text { POT } \\ 1569 & \text { GshA } \\ 1570 & \text { NrtR } \\ 1571 & 51 \\ 1572 & \text { RpsB } \\ 1573 & \text { YaeT } \\ 1574 & \text { pp } \\ 1575 & \text { Oxydation } \\ 1576 & \text { Al } \\ 1577 & \text { PQQ } \\ 1578 & \text { NifN } \\ 1579 & \text { aconitate } \\ 1580 & \text { haloacid } \\ 1581 & \text { betaine } \\ 1582 & \text { transketolase } \\ 1583 & \text { lateral } \\ 1584 & \text { Oxalate } \\ 1585 & \text { med } \\ 1586 & \text { TenA } \\ 1587 & \text { OxyR } \\ 1588 & \text { Phaz } \\ 1589 & \text { TyrR } \\ 1590 & \text { PMM } \\ 1591 & \text { heterodisulfide } \\ 1592 & \text { TPR } \\ 1593 & \text { trigger } \\ 1594 & \text { MaoC } \\ 1595 & \text { enterobactin } \\ 1596 & \text { CspR } \\ 1597 & \text { BlpY } \\ 1598 & \text { OMPP1 } \\ 1599 & \text { STPK } \\ 1600 & \text { Cyp130 } \\ 1601 & \text { FadL } \\ 1602 & \text { l30 } \\ 1603 & \text { TodX } \\ 1604 & \text { ggppsase } \\ 1605 & \text { GGPP } \\ 1606 & \text { GadB } \\ 1607 & \text { FPP } \\ 1608 & \text { IdsA1 } \\ 1609 & \text { prenyltransferase } \\ 1610 & \text { PHYA } \\ 1611 & \text { YcCS } \\ 1612 & \text { YhfK } \\ 1613 & \text { intergral } \\ 1614 & \text { flage3llar } \\ 1615 & \text { EngD } \\ 1616 & \text { PrpE } \\ 1617 & \text { NLP } \\ 1618 & \text { S15 } \\ & \\ 1590\end{array}$
0.017029
0.017029
0.017029
0.017029
0.017029
0.017029
0.017029
0.017029
0.017029
0.016972
0.016972
0.016972
0.01686
0.016819
0.016814
0.016768
0.016517
0.016513
0.01641
0.016325
0.016325
0.016325
0.016325
0.016325
0.016325
0.016325
0.0163
0.01627
0.016231
0.016231
0.016168
0.016115
0.016115
0.016115
0.016115
0.016115
0.016115
0.016115
0.016115
0.016115
0.016115
0.016115
0.016115
0.016115
0.016115
0.016115
0.016115
0.016115
0.016115
0.016115
0.016115
0.016115
0.01603
0.01596 


\begin{tabular}{|c|c|c|}
\hline 1619 & pentapeptide & 0.015844 \\
\hline 1620 & NusA & 0.015772 \\
\hline 1621 & $\mathrm{PPE}$ & 0.015739 \\
\hline 1622 & IS66 & 0.015684 \\
\hline 1623 & methanol & 0.015679 \\
\hline 1624 & dnaB & 0.015679 \\
\hline 1625 & CUT2 & 0.015679 \\
\hline 1626 & turn & 0.015586 \\
\hline 1627 & $\mathrm{XRE}$ & 0.015529 \\
\hline 1628 & ion & 0.015491 \\
\hline 1629 & negative & 0.015342 \\
\hline 1630 & thioredoxin & 0.015303 \\
\hline 1631 & IS186 & 0.015201 \\
\hline 1632 & IS 421 & 0.015201 \\
\hline 1633 & EvgS & 0.015201 \\
\hline 1634 & except & 0.015201 \\
\hline 1635 & PPE3 & 0.015201 \\
\hline 1636 & GogA & 0.015201 \\
\hline 1637 & GtgA & 0.015201 \\
\hline 1638 & MdtA & 0.015201 \\
\hline 1639 & $\operatorname{DinI}$ & 0.015201 \\
\hline 1640 & PotA & 0.015201 \\
\hline 1641 & FliH & 0.015201 \\
\hline 1642 & GlyP & 0.015201 \\
\hline 1643 & YjbJ & 0.015201 \\
\hline 1644 & YejF & 0.015201 \\
\hline 1645 & AGCS & 0.015201 \\
\hline 1646 & trasnporter & 0.015201 \\
\hline 1647 & YCE & 0.015201 \\
\hline 1648 & Adh1 & 0.015201 \\
\hline 1649 & TraR & 0.015183 \\
\hline 1650 & DksA & 0.015183 \\
\hline 1651 & mycothiol & 0.015032 \\
\hline 1652 & M2 4 & 0.014993 \\
\hline 1653 & proteolytic & 0.014874 \\
\hline 1654 & hexose & 0.014859 \\
\hline 1655 & starch & 0.014859 \\
\hline 1656 & rich & 0.014824 \\
\hline 1657 & SsrA & 0.014774 \\
\hline 1658 & L35 & 0.014716 \\
\hline 1659 & HemK & 0.014716 \\
\hline 1660 & S7 & 0.014716 \\
\hline 1661 & LSU & 0.014647 \\
\hline 1662 & DctP & 0.014493 \\
\hline 1663 & $\mathrm{Ni}$ & 0.014402 \\
\hline 1664 & apparatus & 0.014402 \\
\hline 1665 & phytase & 0.014386 \\
\hline 1666 & ROCA & 0.014386 \\
\hline 1667 & LysRS & 0.014386 \\
\hline 1668 & orfA & 0.014306 \\
\hline 1669 & patatin & 0.014302 \\
\hline 1670 & $\mathrm{~F} \operatorname{lrC}$ & 0.014286 \\
\hline 1671 & $F \operatorname{lr} B$ & 0.014286 \\
\hline 1672 & FlaL & 0.014286 \\
\hline
\end{tabular}




$\begin{array}{ll}1673 & \text { MetE } \\ 1674 & \text { LipN } \\ 1675 & \text { DrrC } \\ 1676 & \text { DRRB } \\ 1677 & \text { DIM } \\ 1678 & \text { daunorubicin } \\ 1679 & \text { CydC } \\ 1680 & \text { trpB } \\ 1681 & \text { Sxy } \\ 1682 & \text { FtsJ } \\ 1683 & \text { RrmJ } \\ 1684 & \text { m2U2552 } \\ 1685 & \text { IS30 } \\ 1686 & \text { TfoX1 } \\ 1687 & \text { SufC } \\ 1688 & \text { SufBCD } \\ 1689 & \text { SufD } \\ 1690 & \text { ketodeoxygluconokinase } \\ 1691 & \text { UMP } \\ 1692 & \text { ThiH } \\ 1693 & \text { FixG } \\ 1694 & \text { PurT } \\ 1695 & \text { CoM } \\ 1696 & \text { nrdA } \\ 1697 & \text { bIF } \\ 1698 & \text { exoenzyme } \\ 1699 & \text { Acetone } \\ 1700 & \text { AcxA } \\ 1701 & \text { Octylprenyl } \\ 1702 & \text { IspB } \\ 1703 & \text { Hipo } \\ 1704 & \text { hippurate } \\ 1705 & \text { DsrF } \\ 1706 & \text { DsrE } \\ 1707 & \text { molecular } \\ 1708 & \text { xylanase } \\ 1709 & \text { YoeB } \\ 1710 & \text { Txe } \\ 1711 & \text { YajC } \\ 1712 & \text { Ste24 } \\ 1713 & \text { FecR } \\ 1714 & \text { triple } \\ 1715 & \text { primosomal } \\ 1716 & \text { UvrD } \\ 1717 & \text { glyoxylase } \\ 1718 & \text { ModE } \\ 1719 & \text { topoisomerase } \\ 1720 & \text { stage } \\ 1721 & \text { diketo } \\ 1722 & \text { Propanoyl } \\ 1723 & \text { EAL } \\ 1724 & \text { endopeptidase } \\ 1725 & \text { rieske } \\ 1726 & \text { monomer } \\ & \end{array}$

0.014286

0.014286

0.014286

0.014286

0.014286

0.014286

0.014286

0.014286

0.014286

0.014286

0.014286

0.014286

0.014286

0.014286

0.014286

0.014286

0.014286

0.014286

0.014286

0.014286

0.014286

0.014286

0.014286

0.014286

0.014286

0.014286

0.014286

0.014286

0.014286

0.014286

0.014286

0.014286

0.014286

0.014286

0.014193

0.014188

0.014188

0.014188

0.014188

0.014188

0.014188

0.014188

0.014093

0.014093

0.013956

0.013956

0.013952

0.013945

0.013945

0.013904

0.013904

0.013875

0.013863

0.013739 


$\begin{array}{ll}1727 & \text { CbpA } \\ 1728 & \text { b558 } \\ 1729 & \text { didehydrogluconate } \\ 1730 & \text { alkanesulfonate } \\ 1731 & \text { curved } \\ 1732 & \text { M23 } \\ 1733 & \text { agmatinase } \\ 1734 & \text { amidolyase } \\ 1735 & \text { copper } \\ 1736 & \text { polyketide } \\ 1737 & \text { FlgT } \\ 1738 & \text { YhgF } \\ 1739 & \text { AmgS } \\ 1740 & \text { family } \\ 1741 & \text { is982 } \\ 1742 & \text { StbB } \\ 1743 & \text { YbgC } \\ 1744 & \text { YbgF } \\ 1745 & \text { SS } \\ 1746 & \text { Sjoegren } \\ 1747 & \text { syndrome } \\ 1748 & \text { RoRNP } \\ 1749 & \text { Ro } \\ 1750 & \text { RimP } \\ 1751 & \text { IID } \\ 1752 & \text { A2 } \\ 1753 & \text { autoantigen } \\ 1754 & \text { TROVE } \\ 1755 & \text { S6P } \\ 1756 & \text { benzaldehyde } \\ 1757 & \text { MshF } \\ 1758 & \text { MshA } \\ 1759 & \text { energy } \\ 1760 & \text { hyperosmotically } \\ 1761 & \text { taxis } \\ 1762 & \text { NifV } \\ 1763 & \text { YngJ } \\ 1764 & \text { AO } \\ 1765 & \text { AcdA3 } \\ 1766 & \text { LAO } \\ 1767 & \text { purE } \\ 1768 & \text { cleavage } \\ 1769 & \text { rhamnogalacturonase } \\ 1770 & \text { Clps } \\ 1771 & \text { survival } \\ 1772 & \text { preferring } \\ 1773 & \text { ketoadipyl } \\ 1774 & \text { deoxyuronate } \\ 1775 & \text { RstA } \\ 1776 & \text { PilE } \\ 1777 & \text { sulphate } \\ 1778 & \text { Zn2 } \\ 1779 & \text { lolD } \\ 1780 & \text { PspF } \\ & \end{array}$
0.013739
0.013739
0.013739
0.01372
0.01366
0.013605
0.013546
0.013487
0.013425
0.013377
0.013372
0.013372
0.013372
0.013372
0.013372
0.013372
0.013372
0.013372
0.013372
0.013372
0.013372
0.013372
0.013372
0.013372
0.013372
0.013372
0.013372
0.013372
0.013372
0.013372
0.013372
0.013372
0.013372
0.013372
0.013372
0.013372
0.013372
0.013372
0.013372
0.013372
0.013372
0.013264
0.013137
0.013137
0.013137
0.013132
0.013132
0.013132
0.013132
0.013092
0.013092
0.013092
0.013092
0.013092 


\begin{tabular}{|c|c|}
\hline 1781 & hexulose \\
\hline 1782 & Dethiobiotin \\
\hline 1783 & anhydrase \\
\hline 1784 & fold \\
\hline 1785 & uridine \\
\hline 1786 & resolvase \\
\hline 1787 & isoaspartate \\
\hline 1788 & ROK \\
\hline 1789 & ParA \\
\hline 1790 & taurine \\
\hline 1791 & winged \\
\hline 1792 & nitrile \\
\hline 1793 & C550 \\
\hline 1794 & gaba \\
\hline 1795 & hand \\
\hline 1796 & ClpP \\
\hline 1797 & structural \\
\hline 1798 & LTP1 \\
\hline 1799 & CpxR \\
\hline 1800 & Icd2 \\
\hline 1801 & MppA \\
\hline 1802 & pepetidase \\
\hline 1803 & NrfJ \\
\hline 1804 & benzoquinone \\
\hline 1805 & DMK \\
\hline 1806 & SCS \\
\hline 1807 & bis \\
\hline 1808 & levR \\
\hline 1809 & hexapeptide \\
\hline 1810 & DASS \\
\hline 1811 & def \\
\hline 1812 & FMNH2 \\
\hline 1813 & LIV \\
\hline 1814 & pyruvoyl \\
\hline 1815 & SNF2 \\
\hline 1816 & antidote \\
\hline 1817 & M3 7 \\
\hline 1818 & TOBE \\
\hline 1819 & reducing \\
\hline 1820 & PilV \\
\hline 1821 & Rng \\
\hline 1822 & Melibiose \\
\hline 1823 & riboflavin \\
\hline 1824 & formiminoglutamase \\
\hline 1825 & arginase \\
\hline 1826 & quinone \\
\hline 1827 & $\mathrm{ECF}$ \\
\hline 1828 & farnesyltransferase \\
\hline 1829 & E2 \\
\hline 1830 & OppA \\
\hline 1831 & S20 \\
\hline 1832 & TetR \\
\hline 1833 & rubredoxin \\
\hline 1834 & HAMP \\
\hline
\end{tabular}
0.013092
0.013092
0.013088
0.013052
0.012995
0.012971
0.012934
0.012919
0.012806
0.012778
0.012745
0.01269
0.012604
0.012604
0.0126
0.0126
0.012463
0.012458
0.012458
0.012458
0.012458
0.012458
0.012458
0.012458
0.012458
0.012458
0.012458
0.012458
0.012458
0.012458
0.012446
0.012446
0.012446
0.012446
0.012446
0.012446
0.012446
0.012446
0.012446
0.012446
0.012446
0.012446
0.012376
0.012319
0.012319
0.012164
0.012121
0.012116
0.012076
0.012076
0.011963
0.011894
0.011882
0.011882 


$\begin{array}{ll}1835 & \text { RpoS } \\ 1836 & \text { SAM } \\ 1837 & \text { ThiJ } \\ 1838 & \text { HSP } \\ 1839 & \text { DTW } \\ 1840 & \text { Indole } \\ 1841 & \text { deoxyribonuclease } \\ 1842 & \text { peroxiredoxin } \\ 1843 & \text { pantoate } \\ 1844 & \text { C56 } \\ 1845 & \text { glycolate } \\ 1846 & \text { Prephenate } \\ 1847 & \text { enhanced } \\ 1848 & \text { CysZ } \\ 1849 & \text { PtsP } \\ 1850 & \text { thiocytidine } \\ 1851 & \text { TtcA } \\ 1852 & \text { xyldlegf } \\ 1853 & \text { 12TM } \\ 1854 & 4 \text { TM } \\ 1855 & \text { v6 } \\ 1856 & \text { Mom } \\ 1857 & \text { el4 } \\ 1858 & \text { AtmD } \\ 1859 & \text { MEROPS } \\ 1860 & \text { UptE } \\ 1861 & \text { AhpCTSA } \\ 1862 & \text { zipper } \\ 1863 & \text { bzIP } \\ 1864 & \text { Diacyglycerol } \\ 1865 & \text { Fabz } \\ 1866 & \text { AgxT } \\ 1867 & \text { geranylgeranyl } \\ 1868 & \text { S12 } \\ 1869 & \text { polyamine } \\ 1870 & \text { PhoU } \\ 1871 & \text { IX } \\ 1872 & \text { divalent } \\ 1873 & \text { Mrl } \\ 1874 & \text { RodA } \\ 1875 & \text { DO } \\ 1876 & \text { IIABC } \\ 1877 & \text { AmpC } \\ 1878 & \text { AmpD } \\ 1879 & \text { ExoD } \\ 1880 & \text { Choline } \\ 1881 & \text { Gln } \\ 1882 & \text { flavocytochrome } \\ 1883 & \text { S1 } \\ 1884 & \text { L13 } \\ 1885 & \text { Fe2 } \\ 1886 & \text { tellurium } \\ 1887 & \text { AFG1 } \\ 1888 & \text { LevD } \\ & \\ 1850\end{array}$
0.011882
0.011856
0.011853
0.011799
0.011799
0.011799
0.011662
0.011659
0.011548
0.011548
0.011548
0.011548
0.011548
0.011548
0.011548
0.011548
0.011548
0.011548
0.011543
0.011543
0.011543
0.011543
0.011543
0.011543
0.011543
0.011543
0.011543
0.011543
0.011543
0.011543
0.011543
0.011543
0.011501
0.011501
0.01147
0.011201
0.01119
0.011153
0.011153
0.011092
0.011092
0.011092
0.01102
0.01102
0.01102
0.010978
0.010773
0.010744
0.010733
0.010683
0.010683
0.010683
0.010683
0.010629 


\begin{tabular}{|c|c|}
\hline 1889 & cortex \\
\hline 1890 & L19P \\
\hline 1891 & $\mathrm{~T} 1$ \\
\hline 1892 & CueR \\
\hline 1893 & ZK632 \\
\hline 1894 & RIO1 \\
\hline 1895 & RIO \\
\hline 1896 & MJo 444 \\
\hline 1897 & TrpR \\
\hline 1898 & chains \\
\hline 1899 & $\operatorname{vnfE}$ \\
\hline 1900 & regA \\
\hline 1901 & $\mathrm{BkdR}$ \\
\hline 1902 & insulysin \\
\hline 1903 & acylase \\
\hline 1904 & DhaK \\
\hline 1905 & ParE \\
\hline 1906 & IS1016 \\
\hline 1907 & tol \\
\hline 1908 & pal \\
\hline 1909 & osmolarity \\
\hline 1910 & ModB \\
\hline 1911 & b556 \\
\hline 1912 & $\mathrm{PknH}$ \\
\hline 1913 & FlhC \\
\hline 1914 & sulfhydrylase \\
\hline 1915 & DhnA \\
\hline 1916 & $\mathrm{FO}$ \\
\hline 1917 & aminomethyltransferase \\
\hline 1918 & M2 0 \\
\hline 1919 & DoxX \\
\hline 1920 & OmpR \\
\hline 1921 & MreB \\
\hline 1922 & M4 8 \\
\hline 1923 & nitropropane \\
\hline 1924 & heavy \\
\hline 1925 & PfpI \\
\hline 1926 & fusion \\
\hline 1927 & gyrase \\
\hline 1928 & L31 \\
\hline 1929 & FliL \\
\hline 1930 & Obg \\
\hline 1931 & MreC \\
\hline 1932 & $\mathrm{NifU}$ \\
\hline 1933 & ene \\
\hline 1934 & dioic \\
\hline 1935 & PhzF \\
\hline 1936 & Phenazine \\
\hline 1937 & DppB \\
\hline 1938 & Gifsy \\
\hline 1939 & A3 7 \\
\hline 1940 & thiotransferase \\
\hline 1941 & FliG \\
\hline 1942 & CpaB \\
\hline
\end{tabular}
0.010629
0.010629
0.010629
0.010629
0.010629
0.010629
0.010629
0.010629
0.010629
0.010629
0.010629
0.010629
0.010629
0.010629
0.010629
0.010629
0.010629
0.010506
0.010506
0.010506
0.010506
0.010506
0.010506
0.010506
0.010506
0.010506
0.010506
0.010492
0.010445
0.01036
0.01036
0.01036
0.010287
0.010274
0.010274
0.010245
0.010179
0.010179
0.010165
0.010153
0.010123
0.009964
0.009964
0.0099455
0.0098654
0.0098654
0.0098594
0.0098594
0.0098594
0.0098594
0.0098594
0.0098594
0.0098594
0.0098594 


$\begin{array}{ll}1943 & \text { apolipoprotein } \\ 1944 & \text { divergent } \\ 1945 & \text { PstB } \\ 1946 & \text { OmlA } \\ 1947 & \text { SmpA } \\ 1948 & \text { tusA } \\ 1949 & \text { MacA } \\ 1950 & \text { eny1 } \\ 1951 & \text { LytB } \\ 1952 & \text { PilS } \\ 1953 & \text { RplU } \\ 1954 & \text { imino } \\ 1955 & \text { asaR } \\ 1956 & \text { ahyR } \\ 1957 & \text { nifF } \\ 1958 & \text { FldA } \\ 1959 & \text { mercuric } \\ 1960 & \text { yddQ } \\ 1961 & \text { aa3 } \\ 1962 & \text { DUF3442 } \\ 1963 & \text { EutB } \\ 1964 & \text { PleD } \\ 1965 & \text { acCepting } \\ 1966 & \text { allergen } \\ 1967 & \text { polypeptide } \\ 1968 & \text { GreB } \\ 1969 & 24 \\ 1970 & \text { retraction } \\ 1971 & \text { TrmE } \\ 1972 & \text { lolC } \\ 1973 & \text { TfoX } \\ 1974 & \text { CCoG } \\ 1975 & \text { N5 } \\ 1976 & \text { Rrf2 } \\ 1977 & \text { BadM } \\ 1978 & \text { L27 } \\ 1979 & \text { oligo } \\ 1980 & \text { GltS } \\ 1981 & \text { roadblock } \\ 1982 & \text { LC7 } \\ 1983 & \text { LipC } \\ 1984 & \text { CTP } \\ 1985 & \text { Hlx } \\ 1986 & \text { p69 } \\ 1987 & \text { putattive } \\ 1988 & \text { ClpC } \\ 1989 & \text { MecB } \\ 1990 & \text { L13P } \\ 1991 & \text { ISBm2 } \\ 1992 & \text { IS1477 } \\ 1993 & \text { IS1404 } \\ 1994 & \text { GluA } \\ 1995 & \text { GltL } \\ 1996 & \text { C32 } \\ & \end{array}$
0.0098294
0.0097144
0.0097144
0.0097144
0.0097144
0.0097144
0.0097144
0.0097144
0.0097144
0.0097144
0.0097144
0.0097144
0.0097144
0.0097144
0.0097144
0.0097144
0.0097144
0.0097144
0.0097144
0.0097144
0.0097144
0.0097144
0.009652
0.0094616
0.0094564
0.009436
0.0092394
0.0092128
0.0092128
0.0092128
0.0092128
0.0092128
0.0090474
0.0089149
0.0089149
0.008908
0.008908
0.0088
0.0088
0.0088
0.0088
0.0088
0.0088
0.0088
0.0088
0.0088
0.0088
0.0088
0.0088
0.0088
0.0088
0.0088
0.0088
0.0088 


\begin{tabular}{|c|c|}
\hline 1997 & $\mathrm{birA}$ \\
\hline 1998 & movement \\
\hline 1999 & mxaF1 \\
\hline 2000 & ISEC14 \\
\hline 2001 & IsAfe3 \\
\hline 2002 & $\mathrm{XoxF}$ \\
\hline 2003 & $\mathrm{hmw} 2 \mathrm{C}$ \\
\hline 2004 & hmw2a \\
\hline 2005 & hmwla \\
\hline 2006 & trpCF \\
\hline 2007 & Purs \\
\hline 2008 & PqqE \\
\hline 2009 & allophanate \\
\hline 2010 & cyanide \\
\hline 2011 & poly \\
\hline 2012 & adaptor \\
\hline 2013 & collagen \\
\hline 2014 & formylglutathione \\
\hline 2015 & oxobutanoate \\
\hline 2016 & RnfABCDGE \\
\hline 2017 & Elcomponent \\
\hline 2018 & polyribonucleotide \\
\hline 2019 & $\mathrm{DszC}$ \\
\hline 2020 & bmp \\
\hline 2021 & simple \\
\hline 2022 & PotD \\
\hline 2023 & microcin \\
\hline 2024 & Cache \\
\hline 2025 & TatD \\
\hline 2026 & S16 \\
\hline 2027 & Soluble \\
\hline 2028 & RimM \\
\hline 2029 & LRE \\
\hline 2030 & $\operatorname{RnfA}$ \\
\hline 2031 & IS 200 \\
\hline 2032 & phytoene \\
\hline 2033 & D15 \\
\hline 2034 & theronine \\
\hline 2035 & basic \\
\hline 2036 & Fap \\
\hline 2037 & HlyD \\
\hline 2038 & S6 \\
\hline 2039 & mnth \\
\hline 2040 & Fibronectin \\
\hline 2041 & $\mathrm{Co} 2$ \\
\hline 2042 & P4 50 \\
\hline 2043 & multi \\
\hline 2044 & FlaE \\
\hline 2045 & HlyB \\
\hline 2046 & GabP \\
\hline 2047 & lactoferrin \\
\hline 2048 & MoaE \\
\hline 2049 & PilG \\
\hline 2050 & KdsD \\
\hline
\end{tabular}

0.0088
0.0088
0.0088
0.0088
0.0088
0.0088
0.0088
0.0088
0.0088
0.0088
0.0088
0.0088
0.0087702
0.0087702
0.0087394
0.0087294
0.0087294
0.0086567
0.0086384
0.0086384
0.0085662
0.0085662
0.0085662
0.0085662
0.0085662
0.0085662
0.0085662
0.0084926
0.0084926
0.0084245
0.0084209
0.0083801
0.0083801
0.0083801
0.0083801
0.0083801
0.0083801
0.0083801
0.0083166
0.0082294
0.0081491
0.0081192
0.0081192
0.0080004
0.0080004
0.0079621
0.0079491
0.0079196
0.0079196
0.0079196
0.0079196
0.0079196
0.0078856
0.0078856




\begin{tabular}{|c|c|}
\hline 2051 & glycoendo \\
\hline 2052 & staR \\
\hline 2053 & stalk \\
\hline 2054 & FeaR \\
\hline 2055 & enoly \\
\hline 2056 & C552 \\
\hline 2057 & LplT \\
\hline 2058 & TrpE \\
\hline 2059 & Tils \\
\hline 2060 & PurA \\
\hline 2061 & $\mathrm{REC}$ \\
\hline 2062 & $\mathrm{Rp} f \mathrm{G}$ \\
\hline 2063 & PutA \\
\hline 2064 & $\mathrm{PlsX}$ \\
\hline 2065 & $\operatorname{GrcA}$ \\
\hline 2066 & FemA \\
\hline 2067 & YjgF \\
\hline 2068 & autonomous \\
\hline 2069 & Ohr \\
\hline 2070 & guanidinobutyrase \\
\hline 2071 & $\mathrm{M} 48 \mathrm{~B}$ \\
\hline 2072 & yggG \\
\hline 2073 & IISP \\
\hline 2074 & metaphosphatase \\
\hline 2075 & YvbU \\
\hline 2076 & Quino \\
\hline 2077 & RpoB \\
\hline 2078 & gidB \\
\hline 2079 & Arop \\
\hline 2080 & glycogenase \\
\hline 2081 & DJ \\
\hline 2082 & $3 R$ \\
\hline 2083 & $\mathrm{pH}$ \\
\hline 2084 & Isocitrate \\
\hline 2085 & segregation \\
\hline 2086 & chromosome \\
\hline 2087 & $\mathrm{DEAH}$ \\
\hline 2088 & lipase \\
\hline 2089 & $\mathrm{AP}$ \\
\hline 2090 & butanone \\
\hline 2091 & cardiolipin \\
\hline 2092 & naphthoate \\
\hline 2093 & StbE \\
\hline 2094 & attenuation \\
\hline 2095 & hexosaminidase \\
\hline 2096 & endoglucanase \\
\hline 2097 & septation \\
\hline 2098 & vanadium \\
\hline 2099 & $\operatorname{Ecn} A B$ \\
\hline 2100 & member \\
\hline 2101 & endoribonuclease \\
\hline 2102 & PSP \\
\hline 2103 & opine \\
\hline 2104 & Xaa \\
\hline
\end{tabular}
0.0078856
0.0078856
0.0078856
0.0078856
0.0078856
0.0078856
0.0078856
0.0078856
0.0078856
0.0078856
0.0078856
0.0078856
0.0078856
0.0078856
0.0078856
0.0078856
0.0078856
0.0078856
0.0078856
0.0078856
0.0078856
0.0078856
0.0078856
0.0078856
0.0078856
0.0078856
0.0078856
0.0078856
0.0078856
0.0078759
0.0078521
0.0078521
0.0078521
0.0075912
0.0075912
0.0075462
0.0075243
0.0074096
0.0073874
0.0073724
0.0073724
0.0073241
0.0073241
0.007273
0.007273
0.007273
0.007273
0.007273
0.007273
0.007273
0.0071341
0.0071341
0.0070859
0.0070416 


\begin{tabular}{|c|c|}
\hline 2105 & lysozyme \\
\hline 2106 & $\mathrm{CMP}$ \\
\hline 2107 & flgl \\
\hline 2108 & M1 6 \\
\hline 2109 & FOlEA \\
\hline 2110 & MtrB \\
\hline 2111 & UDPphosphate \\
\hline 2112 & xerc \\
\hline 2113 & resuscitation \\
\hline 2114 & PsiE \\
\hline 2115 & Pnp \\
\hline 2116 & opacity \\
\hline 2117 & FldB \\
\hline 2118 & IS285 \\
\hline 2119 & DUF1667 \\
\hline 2120 & SpuD \\
\hline 2121 & PotF1 \\
\hline 2122 & P9 \\
\hline 2123 & Dhas \\
\hline 2124 & Gfo \\
\hline 2125 & MOCA \\
\hline 2126 & Idh \\
\hline 2127 & DSG \\
\hline 2128 & GlyA \\
\hline 2129 & FixR \\
\hline 2130 & CPA2 \\
\hline 2131 & ybaL \\
\hline 2132 & PRAI \\
\hline 2133 & GadW \\
\hline 2134 & GadE \\
\hline 2135 & maltokinase \\
\hline 2136 & MdtF \\
\hline 2137 & $\operatorname{Sir} A$ \\
\hline 2138 & monophosphatase \\
\hline 2139 & homocitrate \\
\hline 2140 & lysophospholipase \\
\hline 2141 & RhlE \\
\hline 2142 & fumarylacetoacetate \\
\hline 2143 & bax \\
\hline 2144 & SurA \\
\hline 2145 & carbamyl \\
\hline 2146 & isoquinoline \\
\hline 2147 & HrpA \\
\hline 2148 & HII \\
\hline 2149 & LON \\
\hline 2150 & $\mathrm{HPA} 2$ \\
\hline 2151 & ribotide \\
\hline 2152 & aminoimidazole \\
\hline 2153 & U61 \\
\hline 2154 & LD \\
\hline 2155 & s4 \\
\hline 2156 & RuvB \\
\hline 2157 & XerD \\
\hline 2158 & CsbD \\
\hline
\end{tabular}
0.0070024
0.0070024
0.0070024
0.006999
0.0069713
0.0069713
0.0069713
0.0069713
0.0069713
0.0069713
0.0069713
0.0069713
0.0069713
0.0069713
0.0069713
0.0069713
0.0069713
0.0069713
0.0069713
0.0069713
0.0069713
0.0069713
0.0069713
0.0069713
0.0069713
0.0069713
0.0069713
0.0069713
0.0069713
0.0069713
0.0069713
0.0069713
0.0067962
0.0067319
0.0066286
0.0066286
0.0066264
0.0066264
0.0066264
0.0066264
0.0066264
0.0066264
0.0066264
0.0066264
0.0066264
0.0066264
0.0066264
0.0066264
0.0066264
0.0066264
0.0066256
0.0065934
0.0065934
0.0065934 


\begin{tabular}{|c|c|}
\hline 2159 & Sterol \\
\hline 2160 & SpoU \\
\hline 2161 & TrmH \\
\hline 2162 & pilin \\
\hline 2163 & $\mathrm{NrdR}$ \\
\hline 2164 & mRNA \\
\hline 2165 & insensitive \\
\hline 2166 & immunogenic \\
\hline 2167 & MsbA \\
\hline 2168 & RecO \\
\hline 2169 & motility \\
\hline 2170 & SSU \\
\hline 2171 & GGDEF \\
\hline 2172 & $A \operatorname{cox}$ \\
\hline 2173 & MiaB \\
\hline 2174 & N1 \\
\hline 2175 & dibenzothiophene \\
\hline 2176 & lagellar \\
\hline 2177 & $\mathrm{HslU}$ \\
\hline 2178 & PafA \\
\hline 2179 & YwmF \\
\hline 2180 & FbaB \\
\hline 2181 & $\mathrm{DME}$ \\
\hline 2182 & exsA \\
\hline 2183 & Args \\
\hline 2184 & LolB \\
\hline 2185 & parallel \\
\hline 2186 & $\mathrm{NudH}$ \\
\hline 2187 & YgdP \\
\hline 2188 & MIP \\
\hline 2189 & SypA \\
\hline 2190 & inosose \\
\hline 2191 & eric1 \\
\hline 2192 & $\mathrm{~T} 2$ \\
\hline 2193 & $\mathrm{C} 22$ \\
\hline 2194 & ahpc2 \\
\hline 2195 & TauT \\
\hline 2196 & $y b f E$ \\
\hline 2197 & natural \\
\hline 2198 & RhtC \\
\hline 2199 & macrophage \\
\hline 2200 & ompK \\
\hline 2201 & N7 \\
\hline 2202 & xylan \\
\hline 2203 & TRm2 0 \\
\hline 2204 & FliB \\
\hline 2205 & FliUV \\
\hline 2206 & LafV \\
\hline 2207 & LmbE \\
\hline 2208 & UreH \\
\hline 2209 & IS1541 \\
\hline 2210 & homodimeric \\
\hline 2211 & biphosphate \\
\hline 2212 & GIY \\
\hline
\end{tabular}
0.0065934
0.0063502
0.0063502
0.006324
0.0062682
0.0062682
0.0062682
0.0062682
0.0062682
0.0062682
0.0062522
0.0062522
0.0061925
0.0061844
0.0061844
0.0061844
0.0061713
0.0060569
0.0060569
0.0060569
0.0060569
0.0060569
0.0060569
0.0060569
0.0060569
0.0060569
0.0060569
0.0060569
0.0060569
0.0060569
0.0060569
0.0060569
0.0060569
0.0060569
0.0060569
0.0060569
0.0060569
0.0060569
0.0060569
0.0060569
0.0060569
0.0060569
0.0060569
0.0060569
0.0060569
0.0060569
0.0060569
0.0060569
0.0059798
0.0059798
0.0059798
0.0059798
0.0059798
0.0059798 


\begin{tabular}{|c|c|}
\hline 2213 & YIG \\
\hline 2214 & Bacitracin \\
\hline 2215 & $\mathrm{BacA}$ \\
\hline 2216 & MrsA \\
\hline 2217 & number \\
\hline 2218 & $\mathrm{HNH}$ \\
\hline 2219 & DEAD \\
\hline 2220 & WrbA \\
\hline 2221 & FliI \\
\hline 2222 & exsB \\
\hline 2223 & anion \\
\hline 2224 & fiber \\
\hline 2225 & aminoethylphosphonate \\
\hline 2226 & $\mathrm{DsbC}$ \\
\hline 2227 & duplication \\
\hline 2228 & 30 \\
\hline 2229 & SMP \\
\hline 2230 & shape \\
\hline 2231 & desaturase \\
\hline 2232 & coupling \\
\hline 2233 & twitching \\
\hline 2234 & Thic \\
\hline 2235 & iditol \\
\hline 2236 & IS407A \\
\hline 2237 & peri \\
\hline 2238 & Rlud \\
\hline 2239 & 43 \\
\hline 2240 & DivJ \\
\hline 2241 & SeqA \\
\hline 2242 & quinoprotein \\
\hline 2243 & morphogenesis \\
\hline 2244 & driven \\
\hline 2245 & DUF16 \\
\hline 2246 & L36 \\
\hline 2247 & S9B \\
\hline 2248 & YCII \\
\hline 2249 & NLPA \\
\hline 2250 & MapA \\
\hline 2251 & YhaG \\
\hline 2252 & liproprotein \\
\hline 2253 & TrpP \\
\hline 2254 & origin \\
\hline 2255 & N5N10 \\
\hline 2256 & YhdH \\
\hline 2257 & CstA \\
\hline 2258 & ISGme 9 \\
\hline 2259 & IS481 \\
\hline 2260 & cytolysin \\
\hline 2261 & ESAT \\
\hline 2262 & EsxM \\
\hline 2263 & $\operatorname{EsxP}$ \\
\hline 2264 & CoZnCd \\
\hline 2265 & PglB \\
\hline 2266 & PglD \\
\hline
\end{tabular}
0.0059798
0.0059798
0.0059798
0.0059798
0.0059798
0.0059798
0.0058048
0.0057754
0.0057754
0.0057402
0.0057402
0.0057402
0.0057402
0.0057402
0.0057141
0.0057141
0.0057141
0.0056755
0.0055662
0.0055055
0.0053663
0.0053663
0.0053332
0.0053332
0.0053332
0.0053332
0.0053332
0.0053332
0.0053332
0.0052568
0.0052568
0.0052568
0.0052568
0.0052123
0.0052123
0.0052123
0.0052123
0.0051425
0.0051425
0.0051425
0.0051425
0.0051425
0.0051425
0.0051425
0.0051425
0.0051425
0.0051425
0.0051425
0.0051425
0.0051425
0.0051425
0.0051425
0.0051425
0.0051425 


\begin{tabular}{|c|c|}
\hline 2267 & $\mathrm{BCfF}$ \\
\hline 2268 & $\operatorname{BcfA}$ \\
\hline 2269 & ydgu \\
\hline 2270 & PdxS4 \\
\hline 2271 & PdxS \\
\hline 2272 & NarX \\
\hline 2273 & BetI \\
\hline 2274 & oxacillin \\
\hline 2275 & Ssud \\
\hline 2276 & fmtc \\
\hline 2277 & alcane \\
\hline 2278 & $\mathrm{HrcV}$ \\
\hline 2279 & hrc \\
\hline 2280 & SerC \\
\hline 2281 & cyochrome \\
\hline 2282 & YxnA \\
\hline 2283 & YegQ \\
\hline 2284 & $\mathrm{EC} 1$ \\
\hline 2285 & acidtransporter \\
\hline 2286 & DpsA \\
\hline 2287 & IS1647 \\
\hline 2288 & units \\
\hline 2289 & tuaE \\
\hline 2290 & teichuronic \\
\hline 2291 & puitative \\
\hline 2292 & PepT \\
\hline 2293 & bleomycin \\
\hline 2294 & manganese \\
\hline 2295 & Tfp \\
\hline 2296 & hemoglobin \\
\hline 2297 & ZipA \\
\hline 2298 & GutQ \\
\hline 2299 & forming \\
\hline 2300 & OrfB \\
\hline 2301 & oxalocrotonate \\
\hline 2302 & debranching \\
\hline 2303 & mostly \\
\hline 2304 & NorM \\
\hline 2305 & alkylphosphonate \\
\hline 2306 & homeostasis \\
\hline 2307 & trifunctional \\
\hline 2308 & collar \\
\hline 2309 & $\mathrm{NrdH}$ \\
\hline 2310 & FliQ \\
\hline 2311 & developmental \\
\hline 2312 & $\operatorname{MinC}$ \\
\hline 2313 & $\operatorname{TrkA}$ \\
\hline 2314 & Tsx \\
\hline 2315 & PnuC \\
\hline 2316 & YChF \\
\hline 2317 & form \\
\hline 2318 & tolerance \\
\hline 2319 & NifL \\
\hline 2320 & quinate \\
\hline
\end{tabular}

0.0051425

0.0051425

0.0051425

0.0051425

0.0051425

0.0051425

0.0051425

0.0051425

0.0051425

0.0051425

0.0051425

0.0051425

0.0051425

0.0051425

0.0051425

0.0051425

0.0051425

0.0051425

0.0051425

0.0051425

0.0051425

0.0051425

0.0051425

0.0051425

0.0051425

0.0051425

0.0050297

0.0049876

0.0049674

0.0049674

0.0047996

0.0047996

0.0047964

0.0047619

0.0047587

0.0047587

0.0046866

0.0046866

0.0046866

0.0046866

0.0046866

0.0046866

0.0046866

0.0046866

0.0046866

0.0046866

0.0046843

0.0046843

0.0046843

0.0046843

0.0046843

0.0046843

0.0046843

0.0046843 


\begin{tabular}{|c|c|}
\hline 2321 & KpsF \\
\hline 2322 & $\operatorname{cof}$ \\
\hline 2323 & glyoxalase \\
\hline 2324 & MarR \\
\hline 2325 & P47K \\
\hline 2326 & S10 \\
\hline 2327 & cyclodiphosphate \\
\hline 2328 & arsenite \\
\hline 2329 & Kef \\
\hline 2330 & pilB \\
\hline 2331 & module \\
\hline 2332 & addiction \\
\hline 2333 & DnaK \\
\hline 2334 & YkzV \\
\hline 2335 & YkpC \\
\hline 2336 & PsrA \\
\hline 2337 & swapped \\
\hline 2338 & YjiB \\
\hline 2339 & homoaconitate \\
\hline 2340 & $\mathrm{Dcu}$ \\
\hline 2341 & S41A \\
\hline 2342 & Nhac \\
\hline 2343 & AtsE \\
\hline 2344 & NahR \\
\hline 2345 & MetR \\
\hline 2346 & arge1 \\
\hline 2347 & PEPCase \\
\hline 2348 & PEPC \\
\hline 2349 & $\mathrm{BcrC}$ \\
\hline 2350 & Benzoyl \\
\hline 2351 & BadD \\
\hline 2352 & YahK \\
\hline 2353 & IS3411 \\
\hline 2354 & isxal1 \\
\hline 2355 & $\mathrm{HgdB}$ \\
\hline 2356 & DUF 8 81 \\
\hline 2357 & Corrin \\
\hline 2358 & $\mathrm{CzCC}$ \\
\hline 2359 & RseA \\
\hline 2360 & porphyrin \\
\hline 2361 & innermembrane \\
\hline 2362 & implicated \\
\hline 2363 & tauc \\
\hline 2364 & PSA \\
\hline 2365 & Puromycin \\
\hline 2366 & $2 \mathrm{R}$ \\
\hline 2367 & $\mathrm{DAPDH}$ \\
\hline 2368 & Amn \\
\hline 2369 & Tnpc \\
\hline 2370 & DinG \\
\hline 2371 & recycling \\
\hline 2372 & PqqC \\
\hline 2373 & Single \\
\hline 2374 & $\mathrm{ACT}$ \\
\hline
\end{tabular}

0.0046843
0.0046843
0.0046488
0.00459
0.0045483
0.0045483
0.0043853
0.0043853
0.0043423
0.0043423
0.004276
0.004276
0.004276

0.0042281

0.0042281

0.0042281

0.0042281

0.0042281

0.0042281

0.0042281

0.0042281

0.0042281

0.0042281

0.0042281

0.0042281

0.0042281

0.0042281

0.0042281

0.0042281

0.0042281

0.0042281

0.0042281

0.0042281

0.0042281

0.0042281

0.0042281

0.0042281

0.0042281

0.0042281

0.0042281

0.0042281

0.0042281

0.0042281

0.0042281

0.0042281

0.0042281

0.0042281

0.0042281

0.0042281

0.0042281

0.0041563

0.0041563

0.004152

0.0041393 


\begin{tabular}{|c|c|}
\hline 2375 & GlgX \\
\hline 2376 & starvation \\
\hline 2377 & $\operatorname{Rad3}$ \\
\hline 2378 & stability \\
\hline 379 & $\mathrm{CzCD}$ \\
\hline 2380 & OHCU \\
\hline 2381 & ferrisiderophore \\
\hline 2382 & $\mathrm{~b} 1$ \\
\hline 2383 & A $24 \mathrm{~A}$ \\
\hline 2384 & PriA \\
\hline 2385 & cellular \\
\hline 2386 & homologs \\
\hline 2387 & Tat \\
\hline 2388 & MsCS \\
\hline 2389 & leader \\
\hline 2390 & Flp \\
\hline 2391 & diester \\
\hline 2392 & cytidylate \\
\hline 2393 & SpoT \\
\hline 2394 & $\operatorname{RelA}$ \\
\hline 2395 & muramoylpentapeptide \\
\hline 2396 & SpoVR \\
\hline 2397 & antimicrobial \\
\hline 2398 & $\mathrm{P} 1$ \\
\hline 2399 & oxoprolinase \\
\hline 2400 & MATE \\
\hline 2401 & plasmid \\
\hline 2402 & PRC \\
\hline 2403 & F420 \\
\hline 2404 & MviN \\
\hline 2405 & mononucleotide \\
\hline 2406 & de \\
\hline 2407 & YjiH \\
\hline 2408 & polyadenylase \\
\hline 2409 & $\mathrm{~N} 10$ \\
\hline 2410 & pectate \\
\hline 2411 & IPP \\
\hline 2412 & quinohemoprotein \\
\hline 2413 & L32 \\
\hline 2414 & Hemo \\
\hline 2415 & thymidylyltransferase \\
\hline 2416 & $\mathrm{~K} 01322$ \\
\hline 2417 & TpF1 \\
\hline 2418 & S4 9 \\
\hline 2419 & Moty \\
\hline 2420 & U7 \\
\hline 2421 & $24 \mathrm{~K}$ \\
\hline 2422 & $\mathrm{Y} 4 \mathrm{vD}$ \\
\hline 2423 & Prxs \\
\hline 2424 & ComA2 \\
\hline 2425 & PhoB \\
\hline 2426 & J2 \\
\hline 2427 & FlaB \\
\hline 2428 & Uropathogenic \\
\hline
\end{tabular}

0.0041393

0.0041146

0.00404

0.00404

0.00404

0.00404

0.00404

0.00404

0.00404

0.00404

0.0040119

0.0040119

0.0039302

0.0038858

0.003885

0.0038784

0.0037303

0.0036943

0.0036283

0.0036283

0.0036283

0.0036283

0.0036283

0.0036283

0.0035845

0.0035814

0.0035487

0.0035422

0.0035411

0.0034278

0.0034278

0.0033934

0.0033934

0.0033934

0.0033934

0.0033934

0.0033934

0.0033934

0.0033934

0.0033934

0.0033594

0.0033138

0.0033138

0.0033138

0.0033138

0.0033138

0.0033138

0.0033138

0.0033138

0.0033138

0.0033138

0.0033138

0.0033138

0.0033138 


\begin{tabular}{|c|c|}
\hline 2429 & PseE \\
\hline 2430 & USP \\
\hline 2431 & EutH \\
\hline 2432 & $\operatorname{Mog} A$ \\
\hline 2433 & YggT \\
\hline 2434 & ExbD \\
\hline 2435 & TolR \\
\hline 2436 & Cols \\
\hline 2437 & ColR \\
\hline 2438 & ISGme 5 \\
\hline 2439 & ISL3 \\
\hline 2440 & $\mathrm{PCnB}$ \\
\hline 2441 & $S 20 P$ \\
\hline 2442 & FleN \\
\hline 2443 & ISGsu4 \\
\hline 2444 & DppF \\
\hline 2445 & other \\
\hline 2446 & Camelysin \\
\hline 2447 & Uxu \\
\hline 2448 & HydH \\
\hline 2449 & YkuI \\
\hline 2450 & trpEGDC \\
\hline 2451 & TrpL \\
\hline 2452 & cyclotransferase \\
\hline 2453 & 100 \\
\hline 2454 & panthothenate \\
\hline 2455 & GlmU \\
\hline 2456 & PilU \\
\hline 2457 & Zwf \\
\hline 2458 & InsB \\
\hline 2459 & InsA \\
\hline 2460 & S7P \\
\hline 2461 & SypI \\
\hline 2462 & thiokinase \\
\hline 2463 & YHS \\
\hline 2464 & sdaB \\
\hline 2465 & SdaA \\
\hline 2466 & GalU \\
\hline 2467 & Cpsy \\
\hline 2468 & $3 B$ \\
\hline 2469 & $\mathrm{C} 17$ \\
\hline 2470 & riboside \\
\hline 2471 & Lys34 \\
\hline 2472 & DkgB \\
\hline 2473 & $\mathrm{poxB}$ \\
\hline 2474 & vinylacetyl \\
\hline 2475 & $A b \pm D$ \\
\hline 2476 & YjeN \\
\hline 2477 & HexR \\
\hline 2478 & capsular \\
\hline 2479 & GrpE \\
\hline 2480 & Pilus \\
\hline 2481 & hemerythrin \\
\hline 2482 & $\operatorname{Mn} 2$ \\
\hline
\end{tabular}
0.0033138
0.0033138
0.0033138
0.0033138
0.0033138
0.0033138
0.0033138
0.0033138
0.0033138
0.0033138
0.0033138
0.0033138
0.0033138
0.0033138
0.0033138
0.0033138
0.0033138
0.0033138
0.0033138
0.0033138
0.0033138
0.0033138
0.0033138
0.0033138
0.0033138
0.0033138
0.0033138
0.0033138
0.0033138
0.0033138
0.0033138
0.0033138
0.0033138
0.0033138
0.0033138
0.0033138
0.0033138
0.0033138
0.0033138
0.0033138
0.0033138
0.0033138
0.0033138
0.0033138
0.0033138
0.0033138
0.0033138
0.0033138
0.0033138
0.0032388
0.0032388
0.0031762
0.0031148
0.0031004 


$\begin{array}{ll}2483 & \text { Asn } \\ 2484 & \text { urocanate } \\ 2485 & \text { Lactoylglutathione } \\ 2486 & \text { sulfite } \\ 2487 & \text { truncated } \\ 2488 & \text { BioY } \\ 2489 & \text { isovaleryl } \\ 2490 & \text { MxiC } \\ 2491 & \text { YopN } \\ 2492 & \text { InvE } \\ 2493 & \text { LcrE } \\ 2494 & \text { DszA } \\ 2495 & \text { Ferroxidase } \\ 2496 & \text { frpA } \\ 2497 & \text { DhaL } \\ 2498 & \text { modifying } \\ 2499 & \text { CsgG } \\ 2500 & \text { SAH } \\ 2501 & \text { MTA } \\ 2502 & \text { orn } \\ 2503 & \text { arg } \\ 2504 & \text { lys } \\ 2505 & \text { RibD } \\ 2506 & \text { linked } \\ 2507 & \text { Pro } \\ 2508 & \text { anthranilate } \\ 2509 & \text { ferritin } \\ 2510 & \text { benzoate } \\ 2511 & \text { Autoinducer } \\ 2512 & \text { Vanillate } \\ 2513 & \text { bn } \\ 2514 & \text { inosine } \\ 2515 & \text { UBA } \\ 2516 & \text { SurE } \\ 2517 & \text { IS4 } \\ 2518 & \text { S9 } \\ 2519 & \text { Anr } \\ 2520 & \text { GMC } \\ 2521 & \text { PspB } \\ 2522 & \text { PspA2 } \\ 2523 & \text { growth } \\ 2524 & \text { SpoIIE } \\ 2525 & \text { napE } \\ 2526 & \text { YciF } \\ 2527 & \text { ADA } \\ 2528 & \text { PqqD } \\ 2529 & \text { S43 } \\ 2530 & \text { YdjC } \\ 2531 & \text { Pbp } \\ 2532 & \text { gppa2 } \\ 2533 & \text { SspD } \\ 2534 & \text { bypass } \\ 2535 & \text { MiaA } \\ 2536 & \text { IS1554 } \\ & \\ 259\end{array}$
0.0031004
0.0029705
0.0029705
0.0029323
0.0029323
0.0027468
0.0027468
0.0027468
0.0027468
0.0027468
0.0027468
0.0027468
0.0027468
0.0027468
0.0027468
0.0027468
0.0027468
0.0027468
0.0027468
0.0027468
0.0027468
0.0027468
0.0027468
0.0027211
0.0026733
0.0026733
0.0026274
0.0025848
0.0025724
0.0025724
0.0025474
0.0025183
0.0025133
0.0025133
0.0025132
0.0025033
0.0023994
0.0023994
0.0023994
0.0023994
0.0023994
0.0023994
0.0023994
0.0023994
0.0023994
0.0023994
0.0023994
0.0023994
0.0023994
0.0023994
0.0023994
0.0023994
0.0023994
0.0023994 


\begin{tabular}{|c|c|}
\hline 2537 & IS1479 \\
\hline 2538 & ISXO1 \\
\hline 2539 & ISPSSY \\
\hline 2540 & $\mathrm{HspH}$ \\
\hline 2541 & CumA \\
\hline 2542 & CttP \\
\hline 2543 & BarA \\
\hline 2544 & BpeR \\
\hline 2545 & BpeA \\
\hline 2546 & Adherence \\
\hline 2547 & Lost \\
\hline 2548 & linker \\
\hline 2549 & Lads \\
\hline 2550 & trichloroethylene \\
\hline 2551 & DUF3 540 \\
\hline 2552 & GP3 \\
\hline 2553 & $\operatorname{Col} \mathrm{A}$ \\
\hline 2554 & MRP \\
\hline 2555 & Microbial \\
\hline 2556 & YpfJ \\
\hline 2557 & InsL \\
\hline 2558 & likeprotein \\
\hline 2559 & TraC \\
\hline 2560 & tet \\
\hline 2561 & PRPP \\
\hline 2562 & spaB \\
\hline 2563 & GYD \\
\hline 2564 & SA1877 \\
\hline 2565 & SA0281 \\
\hline 2566 & cycloisomerase \\
\hline 2567 & maltooligosyl \\
\hline 2568 & gentisate \\
\hline 2569 & NGG1p \\
\hline 2570 & NIF3 \\
\hline 2571 & YbgI \\
\hline 2572 & mannoheptose \\
\hline 2573 & lysylphosphatidylglycerol \\
\hline 2574 & LysX \\
\hline 2575 & ctr \\
\hline 2576 & RCsA \\
\hline 2577 & BLUF \\
\hline 2578 & eukaryotic \\
\hline 2579 & accesory \\
\hline 2580 & DSRB \\
\hline 2581 & dextransucrase \\
\hline 2582 & cmo5U34 \\
\hline 2583 & YegH \\
\hline 2584 & hemagglutinin \\
\hline 2585 & alkyl \\
\hline 2586 & gp16 \\
\hline 2587 & $\mathrm{Mu}$ \\
\hline 2588 & IsCS \\
\hline 2589 & SohB \\
\hline 2590 & atypical \\
\hline
\end{tabular}

0.0023994

0.0023994

0.0023994

0.0023994

0.0023994

0.0023994

0.0023994

0.0023994

0.0023994

0.0023994

0.0023994

0.0023994

0.0023994

0.0023994

0.0023994

0.0023994

0.0023994

0.0023994

0.0023994

0.0023994

0.0023994

0.0023994

0.0023994

0.0023994

0.0023994

0.0023994

0.0023994

0.0023994

0.0023994

0.0023994

0.0023994

0.0023994

0.0023994

0.0023994

0.0023994

0.0023994

0.0023994

0.0023994

0.0023994

0.0023994

0.0023994

0.0023994

0.0023994

0.0023994

0.0023994

0.0023994

0.0023994

0.0022614

0.0021366

0.0021002

0.0021002

0.0021002

0.0021002

0.0021002 


\begin{tabular}{|c|c|}
\hline 2591 & lysis \\
\hline 2592 & UPF0115 \\
\hline 2593 & macroglobulin \\
\hline 2594 & RpoE \\
\hline 2595 & $r \mathrm{bsA}$ \\
\hline 2596 & PPIASE \\
\hline 2597 & DhaT \\
\hline 2598 & J1 \\
\hline 2599 & Eric \\
\hline 2600 & splicing \\
\hline 2601 & GlpP \\
\hline 2602 & SAF \\
\hline 2603 & orotate \\
\hline 2604 & Flid \\
\hline 2605 & flavohemoprotein \\
\hline 2606 & $\mathrm{U} 32$ \\
\hline 2607 & eita \\
\hline 2608 & ChlI \\
\hline 2609 & Superoxide \\
\hline 2610 & Cro \\
\hline 2611 & $\mathrm{HHE}$ \\
\hline 2612 & prepilin \\
\hline 2613 & OsmC \\
\hline 2614 & wall \\
\hline 2615 & DinB \\
\hline 2616 & glucokinase \\
\hline 2617 & THIF \\
\hline 2618 & calcium \\
\hline 2619 & L19 \\
\hline 2620 & PmbA \\
\hline 2621 & xylosidase \\
\hline 2622 & $\mathrm{Cl}$ \\
\hline 2623 & acetamidase \\
\hline 2624 & YjgQ \\
\hline 2625 & YjgP \\
\hline 2626 & KaiC \\
\hline 2627 & circadian \\
\hline 2628 & clock \\
\hline 2629 & cadmium \\
\hline 2630 & aminodeoxychorismate \\
\hline 2631 & TAXI \\
\hline 2632 & Yags \\
\hline 2633 & cognate \\
\hline 2634 & MttC \\
\hline 2635 & EutP \\
\hline 2636 & Polydeoxyribonucleotide \\
\hline 2637 & PFL \\
\hline 2638 & RibA \\
\hline 2639 & YedZ \\
\hline 2640 & LicA \\
\hline 2641 & reguatory \\
\hline 2642 & MdoD \\
\hline 2643 & elop \\
\hline 2644 & orthophosphate \\
\hline
\end{tabular}

0.0021002
0.0021002
0.0021002
0.0021002
0.0021002
0.0021002
0.0021002
0.0021002
0.0021002
0.0021002
0.0021002
0.002056
0.002056
0.002056

0.0020444

0.0020444

0.0020444

0.0020444

0.001938

0.001856

0.0018054

0.0018054

0.0017716

0.0017138

0.0016853

0.0016853

0.0016853

0.0016475

0.0015987

0.0015165

0.0015165

0.0015165

0.0015165

0.0015165

0.0015165

0.0015165

0.0015165

0.0015165

0.0015165

0.0015165

0.0015103

0.001485

0.001485

0.001485

0.001485

0.001485

0.001485

0.001485

0.001485

0.001485

0.001485

0.001485

0.001485

0.001485 


\begin{tabular}{|c|c|}
\hline 2645 & $\mathrm{ICDH}$ \\
\hline 2646 & ptsH \\
\hline 2647 & acetophenone \\
\hline 2648 & $\mathrm{WxL}$ \\
\hline 2649 & SmpB \\
\hline 2650 & Raffinose \\
\hline 2651 & rafR \\
\hline 2652 & UreG \\
\hline 2653 & end \\
\hline 2654 & $\mathrm{bd} 2$ \\
\hline 2655 & IsFtul \\
\hline 2656 & EnvZ \\
\hline 2657 & acylating \\
\hline 2658 & $\operatorname{amiF}$ \\
\hline 2659 & $\mathrm{HsdR}$ \\
\hline 2660 & alcdhyde \\
\hline 2661 & HpnN \\
\hline 2662 & FhcB \\
\hline 2663 & $2 a$ \\
\hline 2664 & modified \\
\hline 2665 & Mga \\
\hline 2666 & YtfM \\
\hline 2667 & URI \\
\hline 2668 & actinomycete \\
\hline 2669 & lyna \\
\hline 2670 & letA \\
\hline 2671 & hexaprenyltranstransferase \\
\hline 2672 & $\operatorname{TrxA}$ \\
\hline 2673 & DUF3 592 \\
\hline 2674 & $\mathrm{DacC}$ \\
\hline 2675 & pyrBI \\
\hline 2676 & SlyA \\
\hline 2677 & FAA \\
\hline 2678 & $12 \mathrm{~S}$ \\
\hline 2679 & $\operatorname{Sin} I$ \\
\hline 2680 & $y f l J$ \\
\hline 2681 & editing \\
\hline 2682 & lik \\
\hline 2683 & tubulin \\
\hline 2684 & DUF3120 \\
\hline 2685 & ProX \\
\hline 2686 & YCiL \\
\hline 2687 & SilE \\
\hline 2688 & Cucumisin \\
\hline 2689 & ChvD \\
\hline 2690 & YhcM \\
\hline 2691 & tetrameric \\
\hline 2692 & only \\
\hline 2693 & gate \\
\hline 2694 & S33 \\
\hline 2695 & $\operatorname{AnfA}$ \\
\hline 2696 & $\mathrm{PCMT}$ \\
\hline 2697 & srta 3 \\
\hline 2698 & TnpB \\
\hline
\end{tabular}

0.001485

0.001485

0.001485

0.001485

0.001485

0.001485

0.001485

0.001485

0.001485

0.001485

0.001485

0.001485

0.001485

0.001485

0.001485

0.001485

0.001485

0.001485

0.001485

0.001485

0.001485

0.001485

0.001485

0.001485

0.001485

0.001485

0.001485

0.001485

0.001485

0.001485

0.001485

0.001485

0.001485

0.001485

0.001485

0.001485

0.001485

0.001485

0.001485

0.001485

0.001485

0.001485

0.001485

0.001485

0.001485

0.001485

0.001485

0.001485

0.001485

0.001485

0.001485

0.001485

0.001485

0.001485 


\begin{tabular}{|c|c|c|}
\hline 2699 & PrpC & 0.001485 \\
\hline 2700 & CtrA & 0.001485 \\
\hline 2701 & $r c d A$ & 0.001485 \\
\hline 2702 & YxeF & 0.001485 \\
\hline 2703 & PctA & 0.001485 \\
\hline 2704 & ethylmaleimide & 0.001485 \\
\hline 2705 & amido & 0.001485 \\
\hline 2706 & trinitrate & 0.001485 \\
\hline 2707 & TrpG & 0.001485 \\
\hline 2708 & PapD & 0.001485 \\
\hline 2709 & $\operatorname{MvfR}$ & 0.001485 \\
\hline 2710 & silver & 0.0014537 \\
\hline 2711 & IS600 & 0.0014537 \\
\hline 2712 & $\mathrm{ScOl}$ & 0.0014537 \\
\hline 2713 & SenC & 0.0014537 \\
\hline 2714 & cleaving & 0.0014537 \\
\hline 2715 & dimer & 0.0014537 \\
\hline 2716 & AhpC & 0.0014537 \\
\hline 2717 & classes & 0.0014537 \\
\hline 2718 & $\mathrm{VC}$ & 0.0014537 \\
\hline 2719 & $\mathrm{Mn}$ & 0.0014537 \\
\hline 2720 & CapD & 0.0014537 \\
\hline 2721 & Corc & 0.0014537 \\
\hline 2722 & myo & 0.0013982 \\
\hline 2723 & 17 & 0.0012912 \\
\hline 2724 & monovalent & 0.0011646 \\
\hline 2725 & $\mathrm{SEC}$ & 0.0011646 \\
\hline 2726 & tau & 0.0011646 \\
\hline 2727 & L17 & 0.0011415 \\
\hline 2728 & germination & 0.0011415 \\
\hline 2729 & gated & 0.0011415 \\
\hline 2730 & voltage & 0.0011415 \\
\hline 2731 & spore & 0.0010438 \\
\hline 2732 & SMC & 0.0010248 \\
\hline 2733 & FtsZ & 0.0010248 \\
\hline 2734 & promoters & 0.00098849 \\
\hline 2735 & KdpD & 0.00098849 \\
\hline 2736 & UbiE & 0.00098849 \\
\hline 2737 & S18P & 0.00098849 \\
\hline 2738 & Relaxase & 0.00098849 \\
\hline 2739 & FtsK & 0.00096783 \\
\hline 2740 & ArsR & 0.00093754 \\
\hline 2741 & oxygen & 0.00086727 \\
\hline 2742 & invertase & 0.00086727 \\
\hline 2743 & photosystem & 0.00086727 \\
\hline 2744 & Chac & 0.00086727 \\
\hline 2745 & Cys & 0.00081885 \\
\hline 2746 & PilT & 0.00081885 \\
\hline 2747 & hslV & 0.00080706 \\
\hline 2748 & YbiT & 0.00080706 \\
\hline 2749 & analog & 0.00080706 \\
\hline 2750 & mercaptopyruvate & 0.00080706 \\
\hline 2751 & FtsA & 0.00080706 \\
\hline 2752 & OmpW & 0.00080706 \\
\hline
\end{tabular}




\begin{tabular}{|c|c|}
\hline 2753 & CvpA \\
\hline 2754 & RnfD \\
\hline 2755 & xanthosine \\
\hline 2756 & HutH \\
\hline 2757 & $\mathrm{Ku}$ \\
\hline 2758 & ISCmi2 \\
\hline 2759 & $\mathrm{HrpB}$ \\
\hline 2760 & neurotransmitter \\
\hline 2761 & dismutase \\
\hline 2762 & $\mathrm{UTP}$ \\
\hline 2763 & GNAT \\
\hline 2764 & enolase \\
\hline 2765 & L2 8 \\
\hline 2766 & MsrA \\
\hline 2767 & dikinase \\
\hline 2768 & S5 \\
\hline 2769 & tripeptide \\
\hline 2770 & antioxidant \\
\hline 2771 & NlpD \\
\hline 2772 & GvpA \\
\hline 2773 & hupU \\
\hline 2774 & SIR2 \\
\hline 2775 & AioA \\
\hline 2776 & $\operatorname{trp}$ \\
\hline 2777 & YedY \\
\hline 2778 & CysRS \\
\hline 2779 & KleE \\
\hline 2780 & stable \\
\hline 2781 & inheritance \\
\hline 2782 & NadE \\
\hline 2783 & ComM \\
\hline 2784 & osmY \\
\hline 2785 & bodies \\
\hline 2786 & polyhedral \\
\hline 2787 & PduA \\
\hline 2788 & PpiB \\
\hline 2789 & calmodulin \\
\hline 2790 & $\mathrm{~T} 4$ \\
\hline 2791 & Orf 7 \\
\hline 2792 & $\operatorname{CTn} 1$ \\
\hline 2793 & MnhB \\
\hline 2794 & $\operatorname{Tn} 916$ \\
\hline 2795 & energized \\
\hline 2796 & exoDNAse \\
\hline 2797 & TrwC \\
\hline 2798 & $\mathrm{ClCB}$ \\
\hline 2799 & ketothiolase \\
\hline 2800 & L11P \\
\hline 2801 & Toluene \\
\hline 2802 & sulpur \\
\hline 2803 & cassette \\
\hline 2804 & YhbH \\
\hline 2805 & PerM \\
\hline 2806 & EDD \\
\hline
\end{tabular}
0.00080706
0.00080706
0.00080706
0.00080706
0.00080706
0.00080706
0.00080706
0.00080706
0.0007979
0.0007979
0.00069752
0.00068421
0.00068421
0.00068421
0.00068421
0.00068421
0.00065138
0.00064443
0.00057065
0.00057065
0.00057065
0.00057065
0.00057065
0.00057065
0.00057065
0.00057065
0.00057065
0.00057065
0.00057065
0.00057065
0.00057065
0.00057065
0.00057065
0.00057065
0.00057065
0.00057065
0.00057065
0.00057065
0.00057065
0.00057065
0.00057065
0.00057065
0.00057065
0.00057065
0.00057065
0.00057065
0.00057065
0.00057065
0.00057065
0.00057065
0.00057065
0.00057065
0.00057065
0.00057065 


\begin{tabular}{|c|c|}
\hline 2807 & serotype \\
\hline 2808 & Ugd \\
\hline 2809 & $\mathrm{AlgB}$ \\
\hline 2810 & coiled \\
\hline 2811 & $\operatorname{coil}$ \\
\hline 2812 & blasticidin \\
\hline 2813 & FdhA \\
\hline 2814 & PstC \\
\hline 2815 & Phot \\
\hline 2816 & UreC \\
\hline 2817 & $\mathrm{HmsH}$ \\
\hline 2818 & Hmsprotein \\
\hline 2819 & PgaA \\
\hline 2820 & Mt $1 F$ \\
\hline 2821 & polyols \\
\hline 2822 & CitB \\
\hline 2823 & Yjbc \\
\hline 2824 & LonB \\
\hline 2825 & Atld \\
\hline 2826 & $O C C Q$ \\
\hline 2827 & Octopine \\
\hline 2828 & NudE \\
\hline 2829 & FepB \\
\hline 2830 & crotonobetaine \\
\hline 2831 & DeoD \\
\hline 2832 & HepN \\
\hline 2833 & SrtA \\
\hline 2834 & HyaE \\
\hline 2835 & pheromone \\
\hline 2836 & CLsynthase \\
\hline 2837 & BetB \\
\hline 2838 & ArgG \\
\hline 2839 & HslUV \\
\hline 2840 & Kelongation \\
\hline 2841 & ExuT \\
\hline 2842 & Mob1 \\
\hline 2843 & UTRA \\
\hline 2844 & MetQ \\
\hline 2845 & 556 \\
\hline 2846 & G966 \\
\hline 2847 & $y f c N$ \\
\hline 2848 & AdhC \\
\hline 2849 & dicitrate \\
\hline 2850 & facyl \\
\hline 2851 & FadA6 \\
\hline 2852 & $\mathrm{H} 2 \mathrm{TH}$ \\
\hline 2853 & TSase \\
\hline 2854 & DUF 2173 \\
\hline 2855 & ell \\
\hline 2856 & PanB \\
\hline 2857 & likesulfurtransferase \\
\hline 2858 & YuzG \\
\hline 2859 & 42 \\
\hline 2860 & NMT1 \\
\hline
\end{tabular}

0.00057065

0.00057065

0.00057065

0.00057065

0.00057065

0.00057065

0.00057065

0.00057065

0.00057065

0.00057065

0.00057065

0.00057065

0.00057065

0.00057065

0.00057065

0.00057065

0.00057065

0.00057065

0.00057065

0.00057065

0.00057065

0.00057065

0.00057065

0.00057065

0.00057065

0.00057065

0.00057065

0.00057065

0.00057065

0.00057065

0.00057065

0.00057065

0.00057065

0.00057065

0.00057065

0.00057065

0.00057065

0.00057065

0.00057065

0.00057065

0.00057065

0.00057065

0.00057065

0.00057065

0.00057065

0.00057065

0.00057065

0.00057065

0.00057065

0.00057065

0.00057065

0.00057065

0.00057065

0.00051442 


\begin{tabular}{|c|c|}
\hline 2861 & THI5 \\
\hline 2862 & $\mathrm{CI}$ \\
\hline 2863 & Flio \\
\hline 2864 & pirin \\
\hline 2865 & xenobiotic \\
\hline 2866 & acylneuraminate \\
\hline 2867 & Flic \\
\hline 2868 & Queuine \\
\hline 2869 & $\mathrm{Cu}$ \\
\hline 2870 & Biofilm \\
\hline 2871 & 57 \\
\hline 2872 & monosaccharide \\
\hline 2873 & BolA \\
\hline 2874 & bile \\
\hline 2875 & RpoD \\
\hline 2876 & MgtC \\
\hline 2877 & quinol \\
\hline 2878 & s21 \\
\hline 2879 & LigB \\
\hline 2880 & colanic \\
\hline 2881 & $\operatorname{CcdA}$ \\
\hline 2882 & catechol \\
\hline 2883 & ArdC \\
\hline 2884 & TfdA \\
\hline 2885 & GppA \\
\hline 2886 & Ppx \\
\hline 2887 & dolichol \\
\hline 2888 & IS2606 \\
\hline 2889 & $\mathrm{~m} 41$ \\
\hline 2890 & aciid \\
\hline 2891 & chymotrypsin \\
\hline 2892 & GYP \\
\hline 2893 & $\mathrm{HD}$ \\
\hline 2894 & head \\
\hline 2895 & PoxA \\
\hline 2896 & porphobilinogen \\
\hline 2897 & catalase \\
\hline 2898 & ankyrin \\
\hline 2899 & $\mathrm{HisH}$ \\
\hline 2900 & REP \\
\hline 2901 & yl \\
\hline 2902 & en \\
\hline 2903 & Holliday \\
\hline 2904 & $\mathrm{bd}$ \\
\hline 2905 & $40 \mathrm{H}$ \\
\hline 2906 & 15 \\
\hline 2907 & thermolysin \\
\hline 2908 & NTP \\
\hline 2909 & $\mathrm{HrpL}$ \\
\hline 2910 & mono \\
\hline 2911 & deiminase \\
\hline 2912 & LexA \\
\hline 2913 & endo \\
\hline 2914 & $\mathrm{~b} 6$ \\
\hline
\end{tabular}




\begin{tabular}{|c|c|}
\hline 2915 & Virulence \\
\hline 2916 & MerR \\
\hline 2917 & extra \\
\hline 2918 & CopY \\
\hline 2919 & osmosensitive \\
\hline 2920 & DnaJ \\
\hline 2921 & Tolc \\
\hline 2922 & IS911 \\
\hline 2923 & IS3 \\
\hline 2924 & VagC \\
\hline 2925 & Suft \\
\hline 2926 & 38 \\
\hline 2927 & RpsD \\
\hline 2928 & $\mathrm{BlC}$ \\
\hline 2929 & lipocalin \\
\hline 2930 & KptA \\
\hline 2931 & $\mathrm{NCS} 2$ \\
\hline 2932 & ketopantoate \\
\hline 2933 & ApbA \\
\hline 2934 & PanE \\
\hline 2935 & SecY \\
\hline 2936 & $\mathrm{~S} 13$ \\
\hline 2937 & XopP \\
\hline 2938 & EI IAcomponent \\
\hline 2939 & lyt \\
\hline 2940 & $\mathrm{BioH}$ \\
\hline 2941 & KatB \\
\hline 2942 & kipI \\
\hline 2943 & autophosphorylation \\
\hline 2944 & cap \\
\hline 2945 & LafB \\
\hline 2946 & subgroup \\
\hline 2947 & LprE \\
\hline 2948 & $\mathrm{BRCT}$ \\
\hline 2949 & malonic \\
\hline 2950 & pyrR \\
\hline 2951 & SufE \\
\hline 2952 & architecture \\
\hline 2953 & YxlA \\
\hline 2954 & irad \\
\hline 2955 & ECOPI \\
\hline 2956 & ModA \\
\hline 2957 & azurin \\
\hline 2958 & composite \\
\hline 2959 & SpoVG \\
\hline 2960 & GluP \\
\hline 2961 & $\operatorname{vnfD}$ \\
\hline 2962 & RepC \\
\hline 2963 & IS431 \\
\hline 2964 & exoR \\
\hline 2965 & brake \\
\hline 2966 & YcbD \\
\hline 2967 & FlaN \\
\hline 2968 & polarity \\
\hline
\end{tabular}

$-0.00011686$

$-0.00016534$

$-0.00021829$

$-0.00023031$

$-0.00023031$

$-0.00030882$

$-0.00032577$

$-0.00032577$

$-0.00032577$

$-0.00034373$

$-0.00034373$

$-0.00034373$

$-0.00034373$

$-0.00034373$

$-0.00034373$

$-0.00034373$

$-0.00034373$

$-0.00034373$

$-0.00034373$

$-0.00034373$

$-0.00034373$

$-0.00034373$

$-0.00034373$

$-0.00034373$

$-0.00034373$

$-0.00034373$

$-0.00034373$

$-0.00034373$

$-0.00034373$

$-0.00034373$

$-0.00034373$

$-0.00034373$

$-0.00034373$

$-0.00034373$

$-0.00034373$

$-0.00034373$

$-0.00034373$

$-0.00034373$

$-0.00034373$

$-0.00034373$

$-0.00034373$

$-0.00034373$

$-0.00034373$

$-0.00034373$

$-0.00034373$

$-0.00034373$

$-0.00034373$

$-0.00034373$

$-0.00034373$

$-0.00034373$

$-0.00034373$

$-0.00034373$

$-0.00034373$

$-0.00034373$ 


\begin{tabular}{|c|c|}
\hline 2969 & his \\
\hline 2970 & HasI \\
\hline 2971 & I2 \\
\hline 2972 & acyclic \\
\hline 2973 & Atur \\
\hline 2974 & terpenes \\
\hline 2975 & stearoyl \\
\hline 2976 & CatR \\
\hline 2977 & Endopolygalacturonase \\
\hline 2978 & MdcA \\
\hline 2979 & HasR \\
\hline 2980 & HemY \\
\hline 2981 & AtfA \\
\hline 2982 & CobG \\
\hline 2983 & FadD29 \\
\hline 2984 & DACB2 \\
\hline 2985 & lppK \\
\hline 2986 & KpsF3 \\
\hline 2987 & YveA \\
\hline 2988 & ECsA \\
\hline 2989 & PhnA \\
\hline 2990 & FtsN \\
\hline 2991 & $\mathrm{RfaH}$ \\
\hline 2992 & enzymatic \\
\hline 2993 & DHA2 \\
\hline 2994 & plopB \\
\hline 2995 & YgeY \\
\hline 2996 & DapE \\
\hline 2997 & Оррв \\
\hline 2998 & lipo \\
\hline 2999 & ArgP \\
\hline 3000 & DGQHR \\
\hline 3001 & nat \\
\hline 3002 & arylamine \\
\hline 3003 & YD \\
\hline 3004 & Thrs \\
\hline 3005 & lpqV \\
\hline 3006 & morphine \\
\hline 3007 & SigB \\
\hline 3008 & Hda \\
\hline 3009 & DnaA \\
\hline 3010 & NasR \\
\hline 3011 & yobA \\
\hline 3012 & AcrF \\
\hline 3013 & $A c r B$ \\
\hline 3014 & AcrD \\
\hline 3015 & DmpK \\
\hline 3016 & FhuA \\
\hline 3017 & SoxA \\
\hline 3018 & SnaA \\
\hline 3019 & NtaA \\
\hline 3020 & yutd \\
\hline 3021 & NhaB \\
\hline 3022 & L25p \\
\hline
\end{tabular}

$-0.00034373$

$-0.00034373$

$-0.00034373$

$-0.00034373$

$-0.00034373$

$-0.00034373$

$-0.00034373$

$-0.00034373$

$-0.00034373$

$-0.00034373$

$-0.00034373$

$-0.00034373$

$-0.00034373$

$-0.00034373$

$-0.00034373$

$-0.00034373$

$-0.00034373$

$-0.00034373$

$-0.00034373$

$-0.00034373$

$-0.00034373$

$-0.00034373$

$-0.00034373$

$-0.00034373$

$-0.00034373$

$-0.00034373$

$-0.00034373$

$-0.00034373$

$-0.00034373$

$-0.00034373$

$-0.00034373$

$-0.00034373$

$-0.00034373$

$-0.00034373$

$-0.00034373$

$-0.00034373$

$-0.00034373$

$-0.00034373$

$-0.00034373$

$-0.00034373$

$-0.00034373$

$-0.00034373$

$-0.00034373$

$-0.00034373$

$-0.00034373$

$-0.00034373$

$-0.00034373$

$-0.00034373$

$-0.00034373$

$-0.00034373$

$-0.00034373$

$-0.00034373$

$-0.00034373$

$-0.00034373$ 


\begin{tabular}{|c|c|}
\hline 3023 & DppC \\
\hline 3024 & YeeV \\
\hline 3025 & YeeU \\
\hline 3026 & dienoyl \\
\hline 3027 & $\mathrm{MbtH}$ \\
\hline 3028 & enterochelin \\
\hline 3029 & GpJ \\
\hline 3030 & baseplate \\
\hline 3031 & intron \\
\hline 3032 & ISStmaD4 \\
\hline 3033 & FliT \\
\hline 3034 & cyd \\
\hline 3035 & YbgT \\
\hline 3036 & $\mathrm{ADT}$ \\
\hline 3037 & ExuR \\
\hline 3038 & occlusion \\
\hline 3039 & OlsB \\
\hline 3040 & $\mathrm{Crc}$ \\
\hline 3041 & Lsa \\
\hline 3042 & IC \\
\hline 3043 & Prrc \\
\hline 3044 & $\mathrm{BCCT}$ \\
\hline 3045 & spanning \\
\hline 3046 & Isopentenyl \\
\hline 3047 & $\operatorname{RelE}$ \\
\hline 3048 & msrB \\
\hline 3049 & NHL \\
\hline 3050 & enzymes \\
\hline 3051 & DUF1236 \\
\hline 3052 & LysA \\
\hline 3053 & KdgR \\
\hline 3054 & GcvT \\
\hline 3055 & carbinolamine \\
\hline 3056 & HupG \\
\hline 3057 & OYE \\
\hline 3058 & glucuronosyltransferase \\
\hline 3059 & hemolytic \\
\hline 3060 & allosteric \\
\hline 3061 & U1939 \\
\hline 3062 & $\mathrm{ClC}$ \\
\hline 3063 & Hypc \\
\hline 3064 & production \\
\hline 3065 & SapB \\
\hline 3066 & Streptogrisin \\
\hline 3067 & cupin \\
\hline 3068 & demethylase \\
\hline 3069 & $\mathrm{PhoH}$ \\
\hline 3070 & exosortase \\
\hline 3071 & MoxR \\
\hline 3072 & CP4 \\
\hline 3073 & 90 \\
\hline 3074 & chromate \\
\hline 3075 & U62 \\
\hline 3076 & leucyl \\
\hline
\end{tabular}

$-0.00034373$

$-0.00034373$

$-0.00034373$

$-0.00034373$

$-0.00034373$

$-0.00034373$

$-0.00034373$

$-0.00034373$

$-0.00034373$

$-0.00034373$

$-0.00034373$

$-0.00034373$

$-0.00034373$

$-0.00034373$

$-0.00034373$

$-0.00034373$

$-0.00034373$

$-0.00034373$

$-0.00034373$

$-0.00034373$

$-0.00034373$

$-0.00034373$

$-0.00035975$

$-0.00042178$

$-0.00048613$

$-0.00048613$

$-0.00048613$

$-0.00048613$

$-0.00048613$

$-0.00048613$

$-0.00048613$

$-0.00048613$

$-0.00048613$

$-0.00048613$

$-0.00048613$

$-0.00048613$

$-0.00048613$

$-0.00048613$

$-0.00048613$

$-0.00048613$

$-0.00048613$

$-0.0005089$

$-0.000564$

$-0.00059542$

$-0.00062344$

$-0.00064917$

$-0.00066318$

$-0.00068756$

$-0.00068756$

$-0.00068756$

$-0.00068756$

$-0.00076876$

$-0.00076876$

$-0.00079791$ 


\begin{tabular}{|c|c|}
\hline 3077 & SecA \\
\hline 3078 & antagonist \\
\hline 3079 & UspA \\
\hline 3080 & PilA \\
\hline 3081 & selective \\
\hline 3082 & HupF \\
\hline 3083 & acylphosphatase \\
\hline 3084 & $\mathrm{xth}$ \\
\hline 3085 & aminolevulinate \\
\hline 3086 & IS2404 \\
\hline 3087 & HSP70 \\
\hline 3088 & required \\
\hline 3089 & detoxification \\
\hline 3090 & ElaB \\
\hline 3091 & mobile \\
\hline 3092 & PhoR \\
\hline 3093 & ribofuranosylaminobenzene \\
\hline 3094 & FtsY \\
\hline 3095 & docking \\
\hline 3096 & ISC \\
\hline 3097 & $\mathrm{C} 2$ \\
\hline 3098 & MOCR \\
\hline 3099 & 20 \\
\hline 3100 & 31 \\
\hline 3101 & saccharopine \\
\hline 3102 & YbaK \\
\hline 3103 & GalR \\
\hline 3104 & DppA \\
\hline 3105 & PEBP \\
\hline 3106 & $\mathrm{TM}$ \\
\hline 3107 & FecI \\
\hline 3108 & cytosol \\
\hline 3109 & Crystallin \\
\hline 3110 & diheme \\
\hline 3111 & septum \\
\hline 3112 & $\operatorname{RadA}$ \\
\hline 3113 & $\mathrm{Cd}$ \\
\hline 3114 & IS605 \\
\hline 3115 & corrinoid \\
\hline 3116 & Sortase \\
\hline 3117 & arginyl \\
\hline 3118 & PpiC \\
\hline 3119 & LpqX \\
\hline 3120 & SoxS \\
\hline 3121 & triesterase \\
\hline 3122 & L12P \\
\hline 3123 & 55 \\
\hline 3124 & entc \\
\hline 3125 & ETA \\
\hline 3126 & Phi \\
\hline 3127 & MurC \\
\hline 3128 & $\mathrm{XkdP}$ \\
\hline 3129 & Pard1 \\
\hline 3130 & FolM \\
\hline
\end{tabular}

$-0.00084218$

$-0.00084218$

$-0.00098635$

$-0.0010875$

$-0.0011234$

$-0.0011234$

$-0.0011234$

$-0.0011234$

$-0.0011234$

$-0.0011234$

$-0.0011234$

$-0.0011234$

$-0.0011234$

$-0.0011327$

$-0.0011327$

$-0.0011327$

$-0.0011327$

$-0.0011327$

$-0.0011327$

$-0.0011327$

$-0.0011327$

$-0.0011327$

$-0.0011327$

$-0.0011327$

$-0.0011327$

$-0.0011327$

$-0.0011327$

$-0.0011327$

$-0.0011327$

$-0.0011327$

$-0.0011327$

$-0.0011327$

$-0.0011448$

$-0.0011448$

$-0.0011778$

$-0.0011778$

$-0.0011778$

$-0.0012156$

$-0.0012156$

$-0.0012156$

$-0.0012554$

$-0.0012554$

$-0.0012581$

$-0.0012581$

$-0.0012581$

$-0.0012581$

$-0.0012581$

$-0.0012581$

$-0.0012581$

$-0.0012581$

$-0.0012581$

$-0.0012581$

$-0.0012581$

$-0.0012581$ 


$\begin{array}{ll}3131 & \text { Gly } \\ 3132 & \text { ribbon } \\ 3133 & \text { aryl } \\ 3134 & \text { TRm21 } \\ 3135 & \text { isoprenylcysteine } \\ 3136 & \text { removing } \\ 3137 & \text { M19 } \\ 3138 & \text { RapA } \\ 3139 & \text { HepA } \\ 3140 & \text { 34 } \\ 3141 & \text { targeting } \\ 3142 & \text { RDD } \\ 3143 & \text { dUTP } \\ 3144 & \text { TCp } \\ 3145 & \text { YbiN } \\ 3146 & \text { PCtC } \\ 3147 & \text { CwlB } \\ 3148 & \text { Cwlu } \\ 3149 & \text { Telomeric } \\ 3150 & \text { sinR } \\ 3151 & \text { CysT } \\ 3152 & 2 B \\ 3153 & \text { eIF } \\ 3154 & \text { GyaR } \\ 3155 & \text { YffH } \\ 3156 & \text { YfCH } \\ 3157 & \text { HtrB } \\ 3158 & \text { TIGR02947 } \\ 3159 & \text { Mevalonate } \\ 3160 & \text { PqiA } \\ 3161 & \text { DuS } \\ 3162 & \text { AlaS } \\ 3163 & \text { GPR1 } \\ 3164 & \text { FUN34 } \\ 3165 & \text { yaaH } \\ 3166 & \text { YdaM } \\ 3167 & \text { tartronate } \\ 3168 & \text { FlrA } \\ 3169 & \text { RmlA } \\ 3170 & \text { hypersensitive } \\ 3171 & \text { GpmB } \\ 3172 & \text { FadE36 } \\ 3173 & \text { SsuC } \\ 3174 & \text { SipR } \\ 3175 & \text { AlgR } \\ 3176 & \text { LytR } \\ 3177 & \text { PedR1 } \\ 3178 & \text { SctT } \\ 3179 & \text { SipA } \\ 3180 & \text { ApbE } \\ 3181 & \text { deoxyxylulose } \\ 3182 & \text { Pilo } \\ 3183 & \text { PilQ } \\ 3184 & \text { stem } \\ 317 & \end{array}$

$-0.0012581$

$-0.0012581$

$-0.0012581$

$-0.0012581$

$-0.0012581$

$-0.0012581$

$-0.0012581$

$-0.0012581$

$-0.0012581$

$-0.0012581$

$-0.0012581$

$-0.0012581$

$-0.0012581$

$-0.0012581$

$-0.0012581$

$-0.0012581$

$-0.0012581$

$-0.0012581$

$-0.0012581$

$-0.0012581$

$-0.0012581$

$-0.0012581$

$-0.0012581$

$-0.0012581$

$-0.0012581$

$-0.0012581$

$-0.0012581$

$-0.0012581$

$-0.0012581$

$-0.0012581$

$-0.0012581$

$-0.0012581$

$-0.0012581$

$-0.0012581$

$-0.0012581$

$-0.0012581$

$-0.0012581$

$-0.0012581$

$-0.0012581$

$-0.0012581$

$-0.0012581$

$-0.0012581$

$-0.0012581$

$-0.0012581$

$-0.0012581$

$-0.0012581$

$-0.0012581$

$-0.0012581$

$-0.0012581$

$-0.0012581$

$-0.0012581$

$-0.0012581$

$-0.0012581$

$-0.0012581$ 


$\begin{array}{ll}3185 & \text { mesenchymal } \\ 3186 & \text { UpsA } \\ 3187 & \text { DSCD75 } \\ 3188 & \text { bauD } \\ 3189 & \text { acinetobactin } \\ 3190 & \text { GlcD } \\ 3191 & \text { myosin } \\ 3192 & \text { ecotin } \\ 3193 & \text { RihC } \\ 3194 & \text { M3 } \\ 3195 & \text { YtbI } \\ 3196 & \text { acylhomoserine } \\ 3197 & \text { PIG3 } \\ 3198 & \text { pepF } \\ 3199 & \text { gammas } \\ 3200 & \text { AceK } \\ 3201 & \text { OprB } \\ 3202 & \text { kup } \\ 3203 & \text { ClpXP } \\ 3204 & \text { mannan } \\ 3205 & \text { YwbM } \\ 3206 & \text { pu } \\ 3207 & \text { NirQ } \\ 3208 & \text { denitrification } \\ 3209 & \text { CitMHS } \\ 3210 & \text { SigC } \\ 3211 & \text { lipoic } \\ 3212 & \text { XYlS } \\ 3213 & \text { distant } \\ 3214 & \text { Octamer } \\ 3215 & \text { ice } \\ 3216 & \text { fdxa } \\ 3217 & \text { MoaB } \\ 3218 & \text { GidA } \\ 3219 & \text { nitrous } \\ 3220 & \text { MnmG } \\ 3221 & \text { FdxC } \\ 3222 & \text { HutU } \\ 3223 & \text { hexameric } \\ 3224 & \text { IS1547 } \\ 3225 & \text { CesT } \\ 3226 & \text { FoF1 } \\ 3227 & \text { spmX } \\ 3228 & \text { localization } \\ 3229 & \text { Spas } \\ 3230 & \text { CcmE } \\ 3231 & \text { IVb } \\ 3232 & \text { MutS2 } \\ 3233 & \text { YjdJ } \\ 3234 & \text { YdcK } \\ 3235 & \text { Tn3 } \\ 3236 & \text { proprotein } \\ 3237 & \text { Y4wA } \\ 3238 & \text { isocaprenoyl } \\ & \end{array}$

$-0.0012581$

$-0.0012581$

$-0.0012581$

$-0.0012581$

$-0.0012581$

$-0.0012581$

$-0.0012581$

$-0.0012581$

$-0.0012581$

$-0.0012581$

$-0.0012581$

$-0.0012581$

$-0.0012581$

$-0.0012581$

$-0.0012581$

$-0.0012581$

$-0.0012581$

$-0.0012581$

$-0.0012581$

$-0.0012581$

$-0.0012581$

$-0.0012581$

$-0.0012581$

$-0.0012581$

$-0.0012581$

$-0.0012581$

$-0.0012581$

$-0.0012581$

$-0.0012581$

$-0.0012581$

$-0.0012581$

$-0.0012581$

$-0.0012581$

$-0.0012581$

$-0.0012581$

$-0.0012581$

$-0.0012581$

$-0.0012581$

$-0.0012581$

$-0.0012581$

$-0.0012581$

$-0.0012581$

$-0.0012581$

$-0.0012581$

$-0.0012581$

$-0.0012581$

$-0.0012581$

$-0.0012581$

$-0.0012581$

$-0.0012581$

$-0.0012581$

$-0.0012581$

$-0.0012581$

$-0.0012581$ 


\begin{tabular}{|c|c|}
\hline 3239 & ribazole \\
\hline 3240 & Agas \\
\hline 3241 & hyaluronan \\
\hline 3242 & monoamine \\
\hline 3243 & SigG \\
\hline 3244 & HemL \\
\hline 3245 & $\mathrm{Mca}$ \\
\hline 3246 & $\mathrm{HpcH}$ \\
\hline 3247 & Hpa I \\
\hline 3248 & reponse \\
\hline 3249 & HsfB \\
\hline 3250 & HsfA \\
\hline 3251 & $\mathrm{MlrC}$ \\
\hline 3252 & Ras \\
\hline 3253 & $\mathrm{RCC1}$ \\
\hline 3254 & Pat \\
\hline 3255 & BdhA \\
\hline 3256 & Psts \\
\hline 3257 & $\mathrm{DCC}$ \\
\hline 3258 & TrbB \\
\hline 3259 & PHB \\
\hline 3260 & PpsR \\
\hline 3261 & sulfoacetaldehyde \\
\hline 3262 & $\mathrm{NrfH}$ \\
\hline 3263 & YafE \\
\hline 3264 & YcaC \\
\hline 3265 & BtuB \\
\hline 3266 & Torf \\
\hline 3267 & czccba \\
\hline 3268 & YedW \\
\hline 3269 & Spr \\
\hline 3270 & CobD \\
\hline 3271 & M5 6 \\
\hline 3272 & families \\
\hline 3273 & $\mathrm{PilH}$ \\
\hline 3274 & anminopeptidase \\
\hline 3275 & YsCN \\
\hline 3276 & PomA \\
\hline 3277 & ProQ \\
\hline 3278 & Prop \\
\hline 3279 & PulE \\
\hline 3280 & omp16 \\
\hline 3281 & NnrU \\
\hline 3282 & proten \\
\hline 3283 & Arc \\
\hline 3284 & GlpK2 \\
\hline 3285 & PatA \\
\hline 3286 & disaccharide \\
\hline 3287 & SypP \\
\hline 3288 & DedD \\
\hline 3289 & 11 \\
\hline 3290 & FHA \\
\hline 3291 & ketol \\
\hline 3292 & MdoG \\
\hline
\end{tabular}

$-0.0012581$

$-0.0012581$

$-0.0012581$

$-0.0012581$

$-0.0012581$

$-0.0012581$

$-0.0012581$

$-0.0012581$

$-0.0012581$

$-0.0012581$

$-0.0012581$

$-0.0012581$

$-0.0012581$

$-0.0012581$

$-0.0012581$

$-0.0012581$

$-0.0012581$

$-0.0012581$

$-0.0012581$

$-0.0012581$

$-0.0012581$

$-0.0012581$

$-0.0012581$

$-0.0012581$

$-0.0012581$

$-0.0012581$

$-0.0012581$

$-0.0012581$

$-0.0012581$

$-0.0012581$

$-0.0012581$

$-0.0012581$

$-0.0012581$

$-0.0012581$

$-0.0012581$

$-0.0012581$

$-0.0012581$

$-0.0012581$

$-0.0012581$

$-0.0012581$

$-0.0012581$

$-0.0012581$

$-0.0012581$

$-0.0012581$

$-0.0012581$

$-0.0012581$

$-0.0012581$

$-0.0012581$

$-0.0012581$

$-0.0012581$

$-0.0013768$

$-0.001589$

$-0.001589$

$-0.0016021$ 


\begin{tabular}{|c|c|}
\hline 3293 & adducin \\
\hline 3294 & $\mathrm{HxlR}$ \\
\hline 3295 & variant \\
\hline 3296 & dCMP \\
\hline 3297 & S53 \\
\hline 3298 & inorganic \\
\hline 3299 & various \\
\hline 3300 & $\mathrm{~m} 4$ \\
\hline 3301 & trimethylamine \\
\hline 3302 & GLGF \\
\hline 3303 & DHR \\
\hline 3304 & PDZ \\
\hline 3305 & kexin \\
\hline 3306 & sedolisin \\
\hline 3307 & middle \\
\hline 3308 & kynurenine \\
\hline 3309 & PspA \\
\hline 3310 & This \\
\hline 3311 & TldD \\
\hline 3312 & photo \\
\hline 3313 & Taud \\
\hline 3314 & microcompartments \\
\hline 3315 & measure \\
\hline 3316 & tape \\
\hline 3317 & Terd \\
\hline 3318 & $\mathrm{C} 5$ \\
\hline 3319 & Gnt \\
\hline 3320 & polyol \\
\hline 3321 & CsrA \\
\hline 3322 & PGA \\
\hline 3323 & HtpX \\
\hline 3324 & amidinotransferase \\
\hline 3325 & $\operatorname{MglB}$ \\
\hline 3326 & $\operatorname{RarA}$ \\
\hline 3327 & DegV \\
\hline 3328 & azoreductase \\
\hline 3329 & AidA \\
\hline 3330 & KynB \\
\hline 3331 & Strain \\
\hline 3332 & SUF \\
\hline 3333 & M61 \\
\hline 3334 & LigA \\
\hline 3335 & alamin \\
\hline 3336 & MetJ \\
\hline 3337 & ketose \\
\hline 3338 & pili \\
\hline 3339 & Mce \\
\hline 3340 & $2 \mathrm{OG}$ \\
\hline 3341 & antirestriction \\
\hline 3342 & center \\
\hline 3343 & junction \\
\hline 3344 & IVSP \\
\hline 3345 & TDT \\
\hline 3346 & Pyrd \\
\hline
\end{tabular}

$-0.0016021$

$-0.0016021$

$-0.0016021$

$-0.0016021$

$-0.0016021$

$-0.0016194$

$-0.0016514$

$-0.0016514$

$-0.0016514$

$-0.0016514$

$-0.0016514$

$-0.0016514$

$-0.0016514$

$-0.0016514$

$-0.0016514$

$-0.0016514$

$-0.0016514$

$-0.0016514$

$-0.0016514$

$-0.0016514$

$-0.0016514$

$-0.0017793$

$-0.0017793$

$-0.0017793$

$-0.0017793$

$-0.0017793$

$-0.0017793$

$-0.0017793$

$-0.0017793$

$-0.0017793$

$-0.0017793$

$-0.0017793$

$-0.0017793$

$-0.0017793$

$-0.0017793$

$-0.0017793$

$-0.0017793$

$-0.0017793$

$-0.0017793$

$-0.0017793$

$-0.0017793$

$-0.0017793$

$-0.0017793$

$-0.0017793$

$-0.0017793$

$-0.0017793$

$-0.0017793$

$-0.0019958$

$-0.0020593$

$-0.0020593$

$-0.0020843$

$-0.0021725$

$-0.0021725$

$-0.0021725$ 


$\begin{array}{ll}3347 & \text { RutC } \\ 3348 & \text { IlvC } \\ 3349 & \text { lo5C1 } \\ 3350 & \text { AcCB } \\ 3351 & \text { BJ } \\ 3352 & \text { cap5A } \\ 3353 & \text { rhizopine } \\ 3354 & \text { SPbeta } \\ 3355 & \text { CotV } \\ 3356 & \text { 6S } \\ 3357 & \text { 6Fe } \\ 3358 & \text { fraction } \\ 3359 & \text { insoluble } \\ 3360 & \text { prismane } \\ 3361 & \text { PmrA } \\ 3362 & \text { Cut3 } \\ 3363 & \text { C1962 } \\ 3364 & \text { RlmI } \\ 3365 & \text { m5C1962 } \\ 3366 & \text { IS904 } \\ 3367 & \text { IS1068 } \\ 3368 & \text { AB } \\ 3369 & \text { ecpC } \\ 3370 & \text { YcaI } \\ 3371 & \text { PeaA } \\ 3372 & \text { ChrA } \\ 3373 & \text { ABCtransporter } \\ 3374 & \text { degenerate } \\ 3375 & \text { pair } \\ 3376 & \text { GH3 } \\ 3377 & \text { GatC } \\ 3378 & \text { 2C } \\ 3379 & \text { PedD } \\ 3380 & \text { hdig } \\ 3381 & \text { CytX } \\ 3382 & \text { MadN } \\ 3383 & \text { pipecolate } \\ 3384 & \text { COactivator } \\ 3385 & \text { CydA } \\ 3386 & \text { Ca2 } \\ 3387 & \text { step } \\ 3388 & \text { CsiE } \\ 3389 & \text { biosysnthesis } \\ 3390 & \text { BHC } \\ 3391 & \text { MT } \\ 3392 & \text { A70 } \\ 3393 & \text { PMT } \\ 3394 & \text { ExoL } \\ 3395 & \text { chi } \\ 3396 & \text { HolC } \\ 3397 & \text { CqsA } \\ 3398 & \text { CAI } \\ 3399 & \text { ferripyochelin } \\ 3400 & \text { MtgC } \\ & \end{array}$

$-0.0021725$

$-0.0021725$

$-0.0021725$

$-0.0021725$

$-0.0021725$

$-0.0021725$

$-0.0021725$

$-0.0021725$

$-0.0021725$

$-0.0021725$

$-0.0021725$

$-0.0021725$

$-0.0021725$

$-0.0021725$

$-0.0021725$

$-0.0021725$

$-0.0021725$

$-0.0021725$

$-0.0021725$

$-0.0021725$

$-0.0021725$

$-0.0021725$

$-0.0021725$

$-0.0021725$

$-0.0021725$

$-0.0021725$

$-0.0021725$

$-0.0021725$

$-0.0021725$

$-0.0021725$

$-0.0021725$

$-0.0021725$

$-0.0021725$

$-0.0021725$

$-0.0021725$

$-0.0021725$

$-0.0021725$

$-0.0021725$

$-0.0021725$

$-0.0021725$

$-0.0021725$

$-0.0021725$

$-0.0021725$

$-0.0021725$

$-0.0021725$

$-0.0021725$

$-0.0021725$

$-0.0021725$

$-0.0021725$

$-0.0021725$

$-0.0021725$

$-0.0021725$

$-0.0021725$

$-0.0021725$ 


$\begin{array}{ll}3401 & \text { alkylated } \\ 3402 & \text { NapD } \\ 3403 & \text { M15A } \\ 3404 & \text { HSP60 } \\ 3405 & \text { trefoil } \\ 3406 & \text { TadE } \\ 3407 & \text { butenyl } \\ 3408 & \text { IleS } \\ 3409 & \text { lignostilbene } \\ 3410 & \text { LPTX } \\ 3411 & \text { PhnB } \\ 3412 & \text { NikM } \\ 3413 & \text { marine } \\ 3414 & \text { Ygfo } \\ 3415 & \text { YrbE } \\ 3416 & \text { HPI } \\ 3417 & \text { TSA } \\ 3418 & \text { PylB } \\ 3419 & \text { MMPL } \\ 3420 & \text { Calcineurin } \\ 3421 & \text { YecS } \\ 3422 & \text { HPD } \\ 3423 & \text { HPPDase } \\ 3424 & 4 \text { HPPD } \\ 3425 & \text { entry } \\ 3426 & \text { exclusion } \\ 3427 & \text { Cobo } \\ 3428 & \text { Yrinic } \\ 3429 & \text { Cfr } \\ 3430 & \text { S1A } \\ 3431 & 33 \\ 3432 & \text { YhhW } \\ 3433 & \text { qercetin } \\ 3434 & \text { M12A } \\ 3435 & \text { YcgM } \\ 3436 & \text { oligogalacturonide } \\ 3437 & \text { MazG } \\ 3438 & \text { KguT } \\ 3439 & 73 \\ 3440 & \text { SppA } \\ 3441 & 67 K \\ 3442 & \text { Flavanone } \\ 3443 & \text { MXKDX } \\ 3444 & \text { Yggs } \\ 3445 & \text { anticodon } \\ 3446 & \text { AsnA } \\ 3447 & \text { PaaY } \\ 3448 & \text { OsmE } \\ 3449 & \text { MoeA3 } \\ 3450 & \text { MoeA } \\ 3451 & \text { YqgE } \\ 3452 & \text { tcyP } \\ 3453 & \text { MxaR } \\ 3454 & \text { STAS } \\ & \end{array}$

$-0.0021725$

$-0.0021725$

$-0.0021725$

$-0.0021725$

$-0.0021725$

$-0.0021725$

$-0.0021725$

$-0.0021725$

$-0.0021725$

$-0.0021725$

$-0.0021725$

$-0.0021725$

$-0.0021725$

$-0.0021725$

$-0.0021725$

$-0.0021725$

$-0.0021725$

$-0.0021725$

$-0.0021725$

$-0.0021725$

$-0.0021725$

$-0.0021725$

$-0.0021725$

$-0.0021725$

$-0.0021725$

$-0.0021725$

$-0.0021725$

$-0.0021725$

$-0.0021725$

$-0.0021725$

$-0.0021725$

$-0.0021725$

$-0.0021725$

$-0.0021725$

$-0.0021725$

$-0.0021725$

$-0.0021725$

$-0.0021725$

$-0.0021725$

$-0.0021725$

$-0.0021725$

$-0.0021725$

$-0.0021725$

$-0.0021725$

$-0.0021725$

$-0.0021725$

$-0.0021725$

$-0.0021725$

$-0.0021725$

$-0.0021725$

$-0.0021725$

$-0.0021725$

$-0.0021725$

$-0.0021725$ 


$\begin{array}{ll}3455 & \text { VgrGla } \\ 3456 & \text { RumB } \\ 3457 & \text { ImpD } \\ 3458 & \text { Sucraseferredoxin } \\ 3459 & \text { hemopexin } \\ 3460 & \text { GlmR } \\ 3461 & \text { CtaG } \\ 3462 & \text { YihE } \\ 3463 & \text { RspL } \\ 3464 & \text { kbaZ } \\ 3465 & \text { AthL } \\ 3466 & \text { pyrogallol } \\ 3467 & \text { Vicibactin } \\ 3468 & \text { RTX } \\ 3469 & \text { ETC } \\ 3470 & \text { mcbC } \\ 3471 & \text { AcoK } \\ 3472 & \text { LolA } \\ 3473 & \text { AnfU } \\ 3474 & \text { heparinase } \\ 3475 & \text { XylR } \\ 3476 & \text { DmpR } \\ 3477 & \text { sesnor } \\ 3478 & \text { AerP } \\ 3479 & \text { LapB } \\ 3480 & \text { PdhC } \\ 3481 & \text { L15 } \\ 3482 & \text { YacC } \\ 3483 & \text { ModA2 } \\ 3484 & \text { M30 } \\ 3485 & \text { hyicolysin } \\ 3486 & \text { ClpL } \\ 3487 & \text { sulfoquinovose } \\ 3488 & \text { soret } \\ 3489 & \text { split } \\ 3490 & \text { VWFA } \\ 3491 & \text { HyfE } \\ 3492 & \text { AcoR } \\ 3493 & \text { LC } \\ 3494 & \text { hexosyltransferase } \\ 3495 & \text { MexE } \\ 3496 & \text { arabitol } \\ 3497 & \text { PDF } \\ 3498 & \text { Csel } \\ 3499 & \text { NspV } \\ 3500 & \text { extensin } \\ 3501 & \text { trao } \\ 3502 & \text { DicB } \\ 3503 & \text { NolR } \\ 3504 & \text { VirR } \\ 3505 & \text { MBOAT } \\ 3506 & \text { AlgI } \\ 3507 & \text { gpl1 } \\ 3508 & \text { Dcp } \\ & \end{array}$

$-0.0021725$

$-0.0021725$

$-0.0021725$

$-0.0021725$

$-0.0021725$

$-0.0021725$

$-0.0021725$

$-0.0021725$

$-0.0021725$

$-0.0021725$

$-0.0021725$

$-0.0021725$

$-0.0021725$

$-0.0021725$

$-0.0021725$

$-0.0021725$

$-0.0021725$

$-0.0021725$

$-0.0021725$

$-0.0021725$

$-0.0021725$

$-0.0021725$

$-0.0021725$

$-0.0021725$

$-0.0021725$

$-0.0021725$

$-0.0021725$

$-0.0021725$

$-0.0021725$

$-0.0021725$

$-0.0021725$

$-0.0021725$

$-0.0021725$

$-0.0021725$

$-0.0021725$

$-0.0021725$

$-0.0021725$

$-0.0021725$

$-0.0021725$

$-0.0021725$

$-0.0021725$

$-0.0021725$

$-0.0021725$

$-0.0021725$

$-0.0021725$

$-0.0021725$

$-0.0021725$

$-0.0021725$

$-0.0021725$

$-0.0021725$

$-0.0021725$

$-0.0021725$

$-0.0021725$

$-0.0021725$ 


$\begin{array}{ll}3509 & \text { Pgi } \\ 3510 & \text { defense } \\ 3511 & \text { KdpB } \\ 3512 & \text { MexA } \\ 3513 & \text { TrpA } \\ 3514 & \text { CoaA } \\ 3515 & \text { G2251 } \\ 3516 & \text { IS861 } \\ 3517 & \text { L16 } \\ 3518 & \text { GumB } \\ 3519 & \text { azo } \\ 3520 & \text { xanthan } \\ 3521 & \text { dye } \\ 3522 & \text { RluA } \\ 3523 & \text { FdhA1 } \\ 3524 & \text { EhuC } \\ 3525 & \text { P4 } \\ 3526 & \text { RemN } \\ 3527 & \text { Pup } \\ 3528 & \text { spp } \\ 3529 & \text { C13 } \\ 3530 & \text { MORN } \\ 3531 & \text { IstB } \\ 3532 & \text { legumain } \\ 3533 & \text { hupD } \\ 3534 & \text { DUF2317 } \\ 3535 & \text { Cyanobacterial } \\ 3536 & \text { RsmE } \\ 3537 & \text { eicosanoid } \\ 3538 & \text { tRNATyr } \\ 3539 & \text { ShET2 } \\ 3540 & \text { Cdd } \\ 3541 & \text { near } \\ 3542 & \text { torque } \\ 3543 & 45 \\ 3544 & \text { Dopa } \\ 3545 & \text { surfeit } \\ 3546 & \text { permiase } \\ 3547 & \text { aminase } \\ 3548 & \text { CbbX } \\ 3549 & \text { PsaC } \\ 3550 & \text { KatA } \\ 3551 & \text { mcd } \\ 3552 & \text { etherase } \\ 3553 & \text { alkanal } \\ 3554 & \text { PdhR } \\ 3555 & \text { RimJ } \\ 3556 & \text { AEC } \\ 3557 & \text { he } \\ 3558 & \text { RumA } \\ 3559 & \text { DUF1738 } \\ 3560 & \text { CopA } \\ 3561 & \text { Bh } \\ 3562 & \text { MalE } \\ 35 & \\ 3510\end{array}$

$-0.0021725$

$-0.0021725$

$-0.0021725$

$-0.0021725$

$-0.0021725$

$-0.0021725$

$-0.0021725$

$-0.0021725$

$-0.0021725$

$-0.0021725$

$-0.0021725$

$-0.0021725$

$-0.0021725$

$-0.0021725$

$-0.0021725$

$-0.0021725$

$-0.0021725$

$-0.0021725$

$-0.0021725$

$-0.0021725$

$-0.0021725$

$-0.0021725$

$-0.0021725$

$-0.0021725$

$-0.0021725$

$-0.0021725$

$-0.0021725$

$-0.0021725$

$-0.0021725$

$-0.0021725$

$-0.0021725$

$-0.0021725$

$-0.0021725$

$-0.0021725$

$-0.0021725$

$-0.0021725$

$-0.0021725$

$-0.0021725$

$-0.0021725$

$-0.0021725$

$-0.0021725$

$-0.0021725$

$-0.0021725$

$-0.0021725$

$-0.0021725$

$-0.0021725$

$-0.0021725$

$-0.0021725$

$-0.0021725$

$-0.0021725$

$-0.0021725$

$-0.0021725$

$-0.0021725$

$-0.0021725$ 


\begin{tabular}{|c|c|}
\hline 3563 & ISSfl4 \\
\hline 3564 & Int \\
\hline 3565 & LuxC \\
\hline 3566 & PuuB \\
\hline 3567 & Stc \\
\hline 3568 & CoaBC \\
\hline 3569 & BcrA \\
\hline 3570 & BcrD \\
\hline 3571 & BadG \\
\hline 3572 & BadF \\
\hline 3573 & MetN \\
\hline 3574 & MtnA \\
\hline 3575 & thioribose \\
\hline 3576 & Dac \\
\hline 3577 & PeaH \\
\hline 3578 & $\mathrm{M} 12 \mathrm{~B}$ \\
\hline 3579 & matrixin \\
\hline 3580 & adamalysin \\
\hline 3581 & $\mathrm{GlpM}$ \\
\hline 3582 & Bend \\
\hline 3583 & MJ0936 \\
\hline 3584 & thiopurine \\
\hline 3585 & diene \\
\hline 3586 & AlkJ \\
\hline 3587 & VgrG \\
\hline 3588 & UbiC \\
\hline 3589 & MnmA \\
\hline 3590 & НуиА \\
\hline 3591 & FTR1 \\
\hline 3592 & YqxD \\
\hline 3593 & YaiI \\
\hline 3594 & LolE \\
\hline 3595 & Dcm \\
\hline 3596 & organelle \\
\hline 3597 & PsbM \\
\hline 3598 & DcuC \\
\hline 3599 & IS905 \\
\hline 3600 & toxic \\
\hline 3601 & IS21 \\
\hline 3602 & $\operatorname{Irg} 4$ \\
\hline 3603 & Icm \\
\hline 3604 & Dot \\
\hline 3605 & PUA \\
\hline 3606 & CodY \\
\hline 3607 & CheX \\
\hline 3608 & $24 \mathrm{H}$ \\
\hline 3609 & Skp \\
\hline 3610 & AIR \\
\hline 3611 & PepA \\
\hline 3612 & glyxalase \\
\hline 3613 & ChvE \\
\hline 3614 & YusZ \\
\hline 3615 & endonulease \\
\hline 3616 & AraF \\
\hline
\end{tabular}

$-0.0021725$

$-0.0021725$

$-0.0021725$

$-0.0021725$

$-0.0021725$

$-0.0021725$

$-0.0021725$

$-0.0021725$

$-0.0021725$

$-0.0021725$

$-0.0021725$

$-0.0021725$

$-0.0021725$

$-0.0021725$

$-0.0021725$

$-0.0021725$

$-0.0021725$

$-0.0021725$

$-0.0021725$

$-0.0021725$

$-0.0021725$

$-0.0021725$

$-0.0021725$

$-0.0021725$

$-0.0021725$

$-0.0021725$

$-0.0021725$

$-0.0021725$

$-0.0021725$

$-0.0021725$

$-0.0021725$

$-0.0021725$

$-0.0021725$

$-0.0021725$

$-0.0021725$

$-0.0021725$

$-0.0021725$

$-0.0021725$

$-0.0021725$

$-0.0021725$

$-0.0021725$

$-0.0021725$

$-0.0021725$

$-0.0021725$

$-0.0021725$

$-0.0021725$

$-0.0021725$

$-0.0021725$

$-0.0021725$

$-0.0021725$

$-0.0021725$

$-0.0021725$

$-0.0021725$

$-0.0021725$ 


$\begin{array}{ll}3617 & \text { Dld } \\ 3618 & \text { rspA } \\ 3619 & \text { YfmN } \\ 3620 & \text { EpsH } \\ 3621 & \text { holdfast } \\ 3622 & \text { HfaA } \\ 3623 & \text { NRDEF } \\ 3624 & \text { MycP3 } \\ 3625 & \text { Ald } \\ 3626 & \text { SpoVK } \\ 3627 & \text { Cyp153A16 } \\ 3628 & \text { 153Al6 } \\ 3629 & \text { L29 } \\ 3630 & \text { NarU } \\ 3631 & \text { Pin } \\ 3632 & \text { HPr } \\ 3633 & \text { eubacterial } \\ 3634 & \text { Competence } \\ 3635 & \text { NS } \\ 3636 & \text { lipoate } \\ 3637 & \text { FmdB } \\ 3638 & \text { TatA } \\ 3639 & \text { deoxychorismate } \\ 3640 & \text { L34 } \\ 3641 & \text { rhomboid } \\ 3642 & \text { archaeosine } \\ 3643 & \text { ig } \\ 3644 & \text { Sua5 } \\ 3645 & \text { YwlC } \\ 3646 & \text { Ycio } \\ 3647 & \text { YrdC } \\ 3648 & \text { PurR } \\ 3649 & \text { YceI } \\ 3650 & \text { HtpG } \\ 3651 & \text { rraa } \\ 3652 & \text { sh3 } \\ 3653 & \text { RutG } \\ 3654 & \text { grasp } \\ 3655 & \text { YcgR } \\ 3656 & \text { Cobs } \\ 3657 & \text { SstT } \\ 3658 & \text { antifreeze } \\ 3659 & \text { disulphide } \\ 3660 & \text { PqqA } \\ 3661 & \text { medium } \\ 3662 & \text { feruloyl } \\ 3663 & \text { tannase } \\ 3664 & \text { CHAD } \\ 3665 & \text { uronate } \\ 3666 & \text { hexosulose } \\ 3667 & \text { threo } \\ 3668 & \text { RelB } \\ 3669 & \text { ISxcC1 } \\ 3670 & \text { preQ0 } \\ & \end{array}$

$-0.0021725$

$-0.0021725$

$-0.0021725$

$-0.0021725$

$-0.0021725$

$-0.0021725$

$-0.0021725$

$-0.0021725$

$-0.0021725$

$-0.0021725$

$-0.0021725$

$-0.0021725$

$-0.0021725$

$-0.0021725$

$-0.0021793$

$-0.0021793$

$-0.0021793$

$-0.002244$

$-0.0023357$

$-0.0024048$

$-0.0024048$

$-0.0024048$

$-0.0024048$

$-0.0024048$

$-0.0024259$

$-0.0024259$

$-0.0024259$

$-0.0024259$

$-0.0024259$

$-0.0024259$

$-0.0024259$

$-0.0024259$

$-0.0024259$

$-0.0024259$

$-0.0024259$

$-0.0024259$

$-0.0024259$

$-0.0024259$

$-0.0024259$

$-0.0024259$

$-0.0024259$

$-0.0024259$

$-0.0024259$

$-0.0024259$

$-0.0024259$

$-0.0024259$

$-0.0024259$

$-0.0024259$

$-0.0024259$

$-0.0024259$

$-0.0024259$

$-0.0024259$

$-0.0024259$

$-0.0024259$ 


$\begin{array}{ll}3671 & \text { Tir } \\ 3672 & \text { photolyase } \\ 3673 & \text { tetraacyldisaccharide } \\ 3674 & \text { ac } \\ 3675 & \text { creatinase } \\ 3676 & \text { Multimodular } \\ 3677 & \text { cob } \\ 3678 & \text { CorA } \\ 3679 & \text { lantibiotic } \\ 3680 & \text { Stringent } \\ 3681 & \text { polygalacturonase } \\ 3682 & \text { GlnD } \\ 3683 & \text { TatC } \\ 3684 & \text { tagatose } \\ 3685 & \text { mobilization } \\ 3686 & \text { multiple } \\ 3687 & \text { PfkB } \\ 3688 & \text { gas } \\ 3689 & \text { vesicle } \\ 3690 & \text { l3 } \\ 3691 & \text { pepsy } \\ 3692 & \text { cotransporter } \\ 3693 & \text { lptD } \\ 3694 & \text { iminopeptidase } \\ 3695 & \text { Hsp20 } \\ 3696 & \text { la } \\ 3697 & \text { condensation } \\ 3698 & \text { portal } \\ 3699 & \text { conjugation } \\ 3700 & \text { GltX } \\ 3701 & \text { MoeB } \\ 3702 & \text { EngA } \\ 3703 & \text { Dak } \\ 3704 & \text { DegP } \\ 3705 & \text { Cross } \\ 3706 & \text { FliN } \\ 3707 & \text { oligoendopeptidase } \\ 3708 & \text { secb } \\ 3709 & \text { YcsF } \\ 3710 & \text { oligoribonuclease } \\ 3711 & \text { Nidogen } \\ 3712 & \text { Copg } \\ 3713 & \text { mannonate } \\ 3714 & \text { cobw } \\ 3715 & \text { PdxJ } \\ 3716 & \text { TrkH } \\ 3717 & \text { Old } \\ 3718 & \text { citryl } \\ 3719 & \text { RhtB } \\ 3720 & \text { rare } \\ 3721 & \text { PilM } \\ 3722 & \text { LIVCS } \\ 3723 & \text { MloA } \\ 3724 & \text { S5P }\end{array}$

$-0.0024259$

$-0.0024259$

$-0.0024259$

$-0.0025166$

$-0.0025166$

$-0.0025166$

$-0.0027073$

$-0.0027073$

$-0.0027073$

$-0.0027073$

$-0.0027073$

$-0.0027073$

$-0.0027073$

$-0.0027073$

$-0.0027073$

$-0.0027073$

$-0.0027073$

$-0.0027073$

$-0.0027073$

$-0.0027073$

$-0.0027073$

$-0.0027073$

$-0.0027073$

$-0.0027073$

$-0.0027957$

$-0.0029739$

$-0.0029739$

$-0.0029739$

$-0.002984$

$-0.0030725$

$-0.0030725$

$-0.0030725$

$-0.0030725$

$-0.0030725$

$-0.0030725$

$-0.0030725$

$-0.0030725$

$-0.0030725$

$-0.0030725$

$-0.0030725$

$-0.0030725$

$-0.0030725$

$-0.0030725$

$-0.0030725$

$-0.0030725$

$-0.0030725$

$-0.0030725$

$-0.0030725$

$-0.0030725$

$-0.0030725$

$-0.0030725$

$-0.0030725$

$-0.0030725$

$-0.0030725$ 


\begin{tabular}{|c|c|}
\hline 3725 & Pterin \\
\hline 3726 & M2 3B \\
\hline 3727 & SigK \\
\hline 3728 & RpsK \\
\hline 3729 & 27 \\
\hline 3730 & CgtA \\
\hline 3731 & macroH $2 \mathrm{~A} 1$ \\
\hline 3732 & Cbid \\
\hline 3733 & $\mathrm{C} 11$ \\
\hline 3734 & chlamydial \\
\hline 3735 & polymorphic \\
\hline 3736 & Ktr \\
\hline 3737 & Sers \\
\hline 3738 & Undefined \\
\hline 3739 & tapA \\
\hline 3740 & Foldase \\
\hline 3741 & TusD \\
\hline 3742 & PrsA \\
\hline 3743 & $\mathrm{KH}$ \\
\hline 3744 & $\mathrm{CXXCH}$ \\
\hline 3745 & doubled \\
\hline 3746 & NrgB \\
\hline 3747 & Pfk \\
\hline 3748 & RAMP \\
\hline 3749 & AlgU \\
\hline 3750 & Хynв \\
\hline 3751 & HydE \\
\hline 3752 & part 2 \\
\hline 3753 & G6PDH \\
\hline 3754 & $y f l B$ \\
\hline 3755 & protochlorophyllide \\
\hline 3756 & BenE \\
\hline 3757 & SerB \\
\hline 3758 & ThiB \\
\hline 3759 & orf 6 \\
\hline 3760 & granaticin \\
\hline 3761 & gra \\
\hline 3762 & EXOY \\
\hline 3763 & $\operatorname{RegX} 3$ \\
\hline 3764 & YshA \\
\hline 3765 & Fibrobacter \\
\hline 3766 & Fib \\
\hline 3767 & IA \\
\hline 3768 & ED \\
\hline 3769 & having \\
\hline 3770 & third \\
\hline 3771 & $\mathrm{Dx}$ \\
\hline 3772 & DUF3 575 \\
\hline 3773 & L2 7P \\
\hline 3774 & E3 \\
\hline 3775 & cmnm \\
\hline 3776 & mnm \\
\hline 3777 & U3 4 \\
\hline 3778 & aidB \\
\hline
\end{tabular}

$-0.0030825$

$-0.0030825$

$-0.0030868$

$-0.0030868$

$-0.0030868$

$-0.0030868$

$-0.0030868$

$-0.0030868$

$-0.0030868$

$-0.0030868$

$-0.0030868$

$-0.0030868$

$-0.0030868$

$-0.0030868$

$-0.0030868$

$-0.0030868$

$-0.0030868$

$-0.0030868$

$-0.0030868$

$-0.0030868$

$-0.0030868$

$-0.0030868$

$-0.0030868$

$-0.0030868$

$-0.0030868$

$-0.0030868$

$-0.0030868$

$-0.0030868$

$-0.0030868$

$-0.0030868$

$-0.0030868$

$-0.0030868$

$-0.0030868$

$-0.0030868$

$-0.0030868$

$-0.0030868$

$-0.0030868$

$-0.0030868$

$-0.0030868$

$-0.0030868$

$-0.0030868$

$-0.0030868$

$-0.0030868$

$-0.0030868$

$-0.0030868$

$-0.0030868$

$-0.0030868$

$-0.0030868$

$-0.0030868$

$-0.0030868$

$-0.0030868$

$-0.0030868$

$-0.0030868$

$-0.0030868$ 


$\begin{array}{ll}3779 & \text { plant } \\ 3780 & \text { YoaA } \\ 3781 & \text { YidA } \\ 3782 & \text { Y4sB } \\ 3783 & \text { Y4pF } \\ 3784 & \text { Shy23 } \\ 3785 & \text { YxjL } \\ 3786 & \text { Rhizobactin } \\ 3787 & \text { EamA } \\ 3788 & \text { TMS } \\ 3789 & \text { puruvate } \\ 3790 & \text { gp49 } \\ 3791 & \text { SasN } \\ 3792 & \text { Mov34 } \\ 3793 & \text { MPN } \\ 3794 & \text { PAD } \\ 3795 & \text { PpkA } \\ 3796 & \text { glucopyranoside } \\ 3797 & \text { inosityl } \\ 3798 & \text { tuberculin } \\ 3799 & \text { dipicolinate } \\ 3800 & \text { KguE } \\ 3801 & \text { CCOI } \\ 3802 & \text { dGTPase } \\ 3803 & \text { RnfC } \\ 3804 & \text { RhaS } \\ 3805 & \text { rhaBAD } \\ 3806 & \text { HtrA2 } \\ 3807 & \text { ProV } \\ 3808 & \text { CoxM } \\ 3809 & \text { CutM } \\ 3810 & \text { F1 } \\ 3811 & \text { gfCE } \\ 3812 & \text { VIII } \\ 3813 & \text { DivIC } \\ 3814 & \text { gala } \\ 3815 & \text { FarA } \\ 3816 & \text { DUF6 } \\ 3817 & \text { p49 } \\ 3818 & \text { YbfF } \\ 3819 & \text { PecM } \\ 3820 & \text { AmfC } \\ 3821 & \text { HslJ } \\ 3822 & \text { SURF1 } \\ 3823 & \text { Csd5d } \\ 3824 & \text { gp1 } \\ 3825 & \text { Glt } \\ 3826 & \text { Odh } \\ 3827 & \text { SasR } \\ 3828 & \text { DhaM1 } \\ 3829 & \text { exo } \\ 3830 & \text { Some } \\ 3831 & \text { M17 } \\ 3832 & \text { proper } \\ & \end{array}$

$-0.0030868$

$-0.0030868$

$-0.0030868$

$-0.0030868$

$-0.0030868$

$-0.0030868$

$-0.0030868$

$-0.0030868$

$-0.0030868$

$-0.0030868$

$-0.0030868$

$-0.0030868$

$-0.0030868$

$-0.0030868$

$-0.0030868$

$-0.0030868$

$-0.0030868$

$-0.0030868$

$-0.0030868$

$-0.0030868$

$-0.0030868$

$-0.0030868$

$-0.0030868$

$-0.0030868$

$-0.0030868$

$-0.0030868$

$-0.0030868$

$-0.0030868$

$-0.0030868$

$-0.0030868$

$-0.0030868$

$-0.0030868$

$-0.0030868$

$-0.0030868$

$-0.0030868$

$-0.0030868$

$-0.0030868$

$-0.0030868$

$-0.0030868$

$-0.0030868$

$-0.0030868$

$-0.0030868$

$-0.0030868$

$-0.0030868$

$-0.0030868$

$-0.0030868$

$-0.0030868$

$-0.0030868$

$-0.0030868$

$-0.0030868$

$-0.0030868$

$-0.0030868$

$-0.0030868$

$-0.0030868$ 


\begin{tabular}{|c|c|}
\hline 3833 & $\mathrm{C} 40$ \\
\hline 3834 & rusticyanin \\
\hline 3835 & holin \\
\hline 3836 & PilP \\
\hline 3837 & $\operatorname{Reg} B$ \\
\hline 3838 & BNR \\
\hline 3839 & $\mathrm{~m} 75$ \\
\hline 3840 & imelysin \\
\hline 3841 & PrrB \\
\hline 3842 & MobC \\
\hline 3843 & COQ5 \\
\hline 3844 & Dam \\
\hline 3845 & DrsE \\
\hline 3846 & cobalbumin \\
\hline 3847 & flanked \\
\hline 3848 & yfgL \\
\hline 3849 & YqgF \\
\hline 3850 & trafficking \\
\hline 3851 & psi \\
\hline 3852 & SleB \\
\hline 3853 & PhhR \\
\hline 3854 & transription \\
\hline 3855 & s51 \\
\hline 3856 & strictosidine \\
\hline 3857 & LamG \\
\hline 3858 & HisD \\
\hline 3859 & dienelactone \\
\hline 3860 & Gin \\
\hline 3861 & MauE \\
\hline 3862 & pore \\
\hline 3863 & Appr \\
\hline 3864 & DapA \\
\hline 3865 & 550 \\
\hline 3866 & L1p \\
\hline 3867 & L10Ae \\
\hline 3868 & polyferredoxin \\
\hline 3869 & YbiR \\
\hline 3870 & Pyl \\
\hline 3871 & RseB \\
\hline 3872 & MucB \\
\hline 3873 & Vanz \\
\hline 3874 & potF \\
\hline 3875 & OprG \\
\hline 3876 & YbbN \\
\hline 3877 & HrpQ \\
\hline 3878 & $\mathrm{GalcD}$ \\
\hline 3879 & NADB \\
\hline 3880 & Nnrs \\
\hline 3881 & XylG \\
\hline 3882 & Dnrd \\
\hline 3883 & FruR \\
\hline 3884 & L44e \\
\hline 3885 & broad \\
\hline 3886 & 561 \\
\hline
\end{tabular}

$-0.0030868$

$-0.0030868$

$-0.0030868$

$-0.0030868$

$-0.0030868$

$-0.0030868$

$-0.0030868$

$-0.0030868$

$-0.0030868$

$-0.0030868$

$-0.0030868$

$-0.0030868$

$-0.0030868$

$-0.0030868$

$-0.0030868$

$-0.0030868$

$-0.0030868$

$-0.0030868$

$-0.0030868$

$-0.0030868$

$-0.0030868$

$-0.0030868$

$-0.0030868$

$-0.0030868$

$-0.0030868$

$-0.0030868$

$-0.0030868$

$-0.0030868$

$-0.0030868$

$-0.0030868$

$-0.0030868$

$-0.0030868$

$-0.0030868$

$-0.0030868$

$-0.0030868$

$-0.0030868$

$-0.0030868$

$-0.0030868$

$-0.0030868$

$-0.0030868$

$-0.0030868$

$-0.0030868$

$-0.0030868$

$-0.0030868$

$-0.0030868$

$-0.0030868$

$-0.0030868$

$-0.0030868$

$-0.0030868$

$-0.0030868$

$-0.0030868$

$-0.0030868$

$-0.0030868$

$-0.0030868$ 


\begin{tabular}{|c|c|}
\hline 3887 & AGT \\
\hline 3888 & GltR \\
\hline 3889 & PsiF \\
\hline 3890 & DcyD \\
\hline 3891 & yhhN \\
\hline 3892 & Ibp \\
\hline 3893 & Hut I \\
\hline 3894 & CсpA \\
\hline 3895 & Vat \\
\hline 3896 & DUF1217 \\
\hline 3897 & S58 \\
\hline 3898 & DmpA \\
\hline 3899 & LPXTG \\
\hline 3900 & $\mathrm{CaCA}$ \\
\hline 3901 & $\mathrm{Ca}$ \\
\hline 3902 & FUN14 \\
\hline 3903 & DUF1599 \\
\hline 3904 & S54 \\
\hline 3905 & $\operatorname{Rrx} 2$ \\
\hline 3906 & HpaG2 \\
\hline 3907 & Vsr \\
\hline 3908 & $27 \mathrm{~A}$ \\
\hline 3909 & YNFM \\
\hline 3910 & ImpI \\
\hline 3911 & TagF \\
\hline 3912 & DUF2 874 \\
\hline 3913 & former \\
\hline 3914 & fasciclin \\
\hline 3915 & YajL \\
\hline 3916 & Hsp31 \\
\hline 3917 & h3 \\
\hline 3918 & HemP \\
\hline 3919 & parkinsonism \\
\hline 3920 & cone \\
\hline 3921 & UreJ \\
\hline 3922 & HupE \\
\hline 3923 & fimb \\
\hline 3924 & N2N2 \\
\hline 3925 & vinyl \\
\hline 3926 & $4 \mathrm{VR}$ \\
\hline 3927 & $S 26 B$ \\
\hline 3928 & S26A \\
\hline 3929 & $\mathrm{~S} 24$ \\
\hline 3930 & organocation \\
\hline 3931 & Virb6 \\
\hline 3932 & PucR \\
\hline 3933 & VirB2 \\
\hline 3934 & SusD \\
\hline 3935 & IB \\
\hline 3936 & DUF1713 \\
\hline 3937 & SPOA \\
\hline 3938 & ACR3 \\
\hline 3939 & $\operatorname{TrmA}$ \\
\hline 3940 & oxopent \\
\hline
\end{tabular}

$-0.0030868$

$-0.0030868$

$-0.0030868$

$-0.0030868$

$-0.0030868$

$-0.0030868$

$-0.0030868$

$-0.0030868$

$-0.0030868$

$-0.0030868$

$-0.0030868$

$-0.0030868$

$-0.0030868$

$-0.0030868$

$-0.0030868$

$-0.0030868$

$-0.0030868$

$-0.0030868$

$-0.0030868$

$-0.0030868$

$-0.0030868$

$-0.0030868$

$-0.0030868$

$-0.0030868$

$-0.0030868$

$-0.0030868$

$-0.0030868$

$-0.0030868$

$-0.0030868$

$-0.0030868$

$-0.0030868$

$-0.0030868$

$-0.0030868$

$-0.0030868$

$-0.0030868$

$-0.0030868$

$-0.0030868$

$-0.0030868$

$-0.0030868$

$-0.0030868$

$-0.0030868$

$-0.0030868$

$-0.0030868$

$-0.0030868$

$-0.0030868$

$-0.0030868$

$-0.0030868$

$-0.0030868$

$-0.0030868$

$-0.0030868$

$-0.0030868$

$-0.0030868$

$-0.0030868$

$-0.0030868$ 


$\begin{array}{ll}3941 & \text { mdlA } \\ 3942 & \text { YdfJ } \\ 3943 & \text { YcCT } \\ 3944 & \text { SAICAR } \\ 3945 & \text { ade1 } \\ 3946 & \text { pur7 } \\ 3947 & \text { fucosidase } \\ 3948 & \text { S16P } \\ 3949 & \text { Caffeoyl } \\ 3950 & \text { IS1354 } \\ 3951 & \text { amd } \\ 3952 & \text { MdtJ } \\ 3953 & \text { band } \\ 3954 & \text { MotA3 } \\ 3955 & \text { CdaR } \\ 3956 & \text { CUT1 } \\ 3957 & \text { iso } \\ 3958 & \text { within } \\ 3959 & \text { BAIF } \\ 3960 & \text { GspD } \\ 3961 & \text { YhnG } \\ 3962 & \text { AglR } \\ 3963 & \text { Fha1 } \\ 3964 & \text { SsDNA } \\ 3965 & \text { COmigratory } \\ 3966 & \text { DgCB } \\ 3967 & \text { RecJ } \\ 3968 & \text { glxK } \\ 3969 & \text { CitA } \\ 3970 & \text { MntR } \\ 3971 & \text { MttB2 } \\ 3972 & \text { MttB1 } \\ 3973 & \text { DtxR } \\ 3974 & \text { FpvR } \\ 3975 & \text { sCafolding } \\ 3976 & \text { SoxY } \\ 3977 & \text { HpnH } \\ 3978 & \text { TraG } \\ 3979 & \text { TraD } \\ 3980 & \text { Curing } \\ 3981 & \text { MazE } \\ 3982 & \text { Fae } \\ 3983 & \text { glycanase } \\ 3984 & \text { gp229 } \\ 3985 & \text { intrrupted } \\ 3986 & \text { PhsA } \\ 3987 & \text { Taq } \\ 3988 & \text { GlCC } \\ 3989 & \text { Usg } \\ 3990 & \text { NhaR } \\ 3991 & \text { IroE } \\ 3992 & \text { A24 } \\ 3993 & \text { AbgT } \\ 3994 & \text { BioD } \\ & \end{array}$

$-0.0030868$

$-0.0030868$

$-0.0030868$

$-0.0030868$

$-0.0030868$

$-0.0030868$

$-0.0030868$

$-0.0030868$

$-0.0030868$

$-0.0030868$

$-0.0030868$

$-0.0030868$

$-0.0030868$

$-0.0030868$

$-0.0030868$

$-0.0030868$

$-0.0030868$

$-0.0030868$

$-0.0030868$

$-0.0030868$

$-0.0030868$

$-0.0030868$

$-0.0030868$

$-0.0030868$

$-0.0030868$

$-0.0030868$

$-0.0030868$

$-0.0030868$

$-0.0030868$

$-0.0030868$

$-0.0030868$

$-0.0030868$

$-0.0030868$

$-0.0030868$

$-0.0030868$

$-0.0030868$

$-0.0030868$

$-0.0030868$

$-0.0030868$

$-0.0030868$

$-0.0030868$

$-0.0030868$

$-0.0030868$

$-0.0030868$

$-0.0030868$

$-0.0030868$

$-0.0030868$

$-0.0030868$

$-0.0030868$

$-0.0030868$

$-0.0030868$

$-0.0030868$

$-0.0030868$

$-0.0030868$ 


\begin{tabular}{|c|c|}
\hline 3995 & CAAX \\
\hline 3996 & flippase \\
\hline 3997 & MurJ \\
\hline 3998 & YghU \\
\hline 3999 & Mlr3171 \\
\hline 4000 & angiotensin \\
\hline 4001 & xylA \\
\hline 4002 & ISSod13 \\
\hline 4003 & ISBmu 27 \\
\hline 4004 & ISPg1 \\
\hline 4005 & partial \\
\hline 4006 & YapH \\
\hline 4007 & FlhD \\
\hline 4008 & GtrA \\
\hline 4009 & $\mathrm{dATP}$ \\
\hline 4010 & UbiD \\
\hline 4011 & tim44 \\
\hline 4012 & $\mathrm{SAD}$ \\
\hline 4013 & WhiB 7 \\
\hline 4014 & pra \\
\hline 4015 & NrdI \\
\hline 4016 & HyuE \\
\hline 4017 & FadD32 \\
\hline 4018 & FadD \\
\hline 4019 & LprB \\
\hline 4020 & COMEA \\
\hline 4021 & CelD \\
\hline 4022 & hairpin \\
\hline 4023 & PedF \\
\hline 4024 & arylsulfatase \\
\hline 4025 & $\mathrm{PBS}$ \\
\hline 4026 & SnoaL \\
\hline 4027 & NapC \\
\hline 4028 & CM55 \\
\hline 4029 & antitrypsin \\
\hline 4030 & uup \\
\hline 4031 & unique \\
\hline 4032 & CyaY \\
\hline 4033 & donor \\
\hline 4034 & SBP 56 \\
\hline 4035 & $56 \mathrm{kDa}$ \\
\hline 4036 & 67 \\
\hline 4037 & hemimethylated \\
\hline 4038 & DHA1 \\
\hline 4039 & NifD5 \\
\hline 4040 & GumN \\
\hline 4041 & caspase \\
\hline 4042 & ethylaminohydrolase \\
\hline 4043 & MecA \\
\hline 4044 & sulfotransferase \\
\hline 4045 & WGR \\
\hline 4046 & CopT \\
\hline 4047 & QseB \\
\hline 4048 & CadC \\
\hline
\end{tabular}

$-0.0030868$

$-0.0030868$

$-0.0030868$

$-0.0030868$

$-0.0030868$

$-0.0030868$

$-0.0030868$

$-0.0030868$

$-0.0030868$

$-0.0030868$

$-0.0030868$

$-0.0030868$

$-0.0030868$

$-0.0030868$

$-0.0030868$

$-0.0030868$

$-0.0030868$

$-0.0030868$

$-0.0030868$

$-0.0030868$

$-0.0030868$

$-0.0030868$

$-0.0030868$

$-0.0030868$

$-0.0030868$

$-0.0030868$

$-0.0030868$

$-0.0030868$

$-0.0030868$

$-0.0030868$

$-0.0030868$

$-0.0030868$

$-0.0030868$

$-0.0030868$

$-0.0030868$

$-0.0030868$

$-0.0030868$

$-0.0030868$

$-0.0030868$

$-0.0030868$

$-0.0030868$

$-0.0030868$

$-0.0030868$

$-0.0030868$

$-0.0030868$

$-0.0030868$

$-0.0030868$

$-0.0030868$

$-0.0030868$

$-0.0030868$

$-0.0030868$

$-0.0030868$

$-0.0030868$

$-0.0030868$ 


\begin{tabular}{|c|c|}
\hline 4049 & endoprotease \\
\hline 4050 & DegQ \\
\hline 4051 & SWIM \\
\hline 4052 & $\operatorname{RepB}$ \\
\hline 4053 & CoxF \\
\hline 4054 & mutarotase \\
\hline 4055 & GP55 \\
\hline 4056 & IscU \\
\hline 4057 & GPD \\
\hline 4058 & $\mathrm{HhH}$ \\
\hline 4059 & DUF2933 \\
\hline 4060 & MucD \\
\hline 4061 & ISRSO5 \\
\hline 4062 & Scis \\
\hline 4063 & DivIVA \\
\hline 4064 & sun \\
\hline 4065 & npd \\
\hline 4066 & tola \\
\hline 4067 & PbpG \\
\hline 4068 & dioxopentanoate \\
\hline 4069 & pgap1 \\
\hline 4070 & L14 \\
\hline 4071 & AIG2 \\
\hline 4072 & MdoH \\
\hline 4073 & proenzyme \\
\hline 4074 & MBR \\
\hline 4075 & Tspo \\
\hline 4076 & sialophosphoprotein \\
\hline 4077 & dentin \\
\hline 4078 & RimK \\
\hline 4079 & mrmbrane \\
\hline 4080 & oligomer \\
\hline 4081 & EssC \\
\hline 4082 & $\mathrm{Pcp}$ \\
\hline 4083 & $\mathrm{RfbB}$ \\
\hline 4084 & RpmB2 \\
\hline 4085 & LipU \\
\hline 4086 & glft \\
\hline 4087 & malyl \\
\hline 4088 & SprT \\
\hline 4089 & calpastatin \\
\hline 4090 & $\operatorname{slp}$ \\
\hline 4091 & LipL21 \\
\hline 4092 & Xers \\
\hline 4093 & endoflagellar \\
\hline 4094 & $\operatorname{modD}$ \\
\hline 4095 & PrpF \\
\hline 4096 & $\mathrm{Alg} A$ \\
\hline 4097 & FimV \\
\hline 4098 & enolpyruvyl \\
\hline 4099 & LCfB \\
\hline 4100 & Lyts \\
\hline 4101 & GumL \\
\hline 4102 & PO \\
\hline
\end{tabular}

$-0.0030868$

$-0.0030868$

$-0.0030868$

$-0.0030868$

$-0.0030868$

$-0.0030868$

$-0.0030868$

$-0.0030868$

$-0.0030868$

$-0.0030868$

$-0.0030868$

$-0.0030868$

$-0.0030868$

$-0.0030868$

$-0.0030868$

$-0.0030868$

$-0.0030868$

$-0.0030868$

$-0.0030868$

$-0.0030868$

$-0.0030868$

$-0.0030868$

$-0.0030868$

$-0.0030868$

$-0.0030868$

$-0.0030868$

$-0.0030868$

$-0.0030868$

$-0.0030868$

$-0.0030868$

$-0.0030868$

$-0.0030868$

$-0.0030868$

$-0.0030868$

$-0.0030868$

$-0.0030868$

$-0.0030868$

$-0.0030868$

$-0.0030868$

$-0.0030868$

$-0.0030868$

$-0.0030868$

$-0.0030868$

$-0.0030868$

$-0.0030868$

$-0.0030868$

$-0.0030868$

$-0.0030868$

$-0.0030868$

$-0.0030868$

$-0.0030868$

$-0.0030868$

$-0.0030868$

$-0.0030868$ 


\begin{tabular}{|c|c|}
\hline 4103 & $\mathrm{CPxCG}$ \\
\hline 4104 & isolated \\
\hline 4105 & $6 \mathrm{~A}$ \\
\hline 4106 & Yellow \\
\hline 4107 & isoniazid \\
\hline 4108 & IniC \\
\hline 4109 & inductible \\
\hline 4110 & vanw \\
\hline 4111 & WS \\
\hline 4112 & FrzG \\
\hline 4113 & MGAT \\
\hline 4114 & DGAT \\
\hline 4115 & pgac \\
\hline 4116 & argninine \\
\hline 4117 & allo \\
\hline 4118 & DmsD \\
\hline 4119 & SycD \\
\hline 4120 & $\mathrm{LCrH}$ \\
\hline 4121 & PhnG \\
\hline 4122 & TomA0 \\
\hline 4123 & Ompc \\
\hline 4124 & sulphonates \\
\hline 4125 & antimonite \\
\hline 4126 & ArsB \\
\hline 4127 & COQ7 \\
\hline 4128 & UvrA \\
\hline 4129 & diadenosine \\
\hline 4130 & WPhi \\
\hline 4131 & maintenance \\
\hline 4132 & NolW \\
\hline 4133 & ApaG \\
\hline 4134 & GcrA \\
\hline 4135 & cycle \\
\hline 4136 & Fpg \\
\hline 4137 & UptF \\
\hline 4138 & $\operatorname{ErpA}$ \\
\hline 4139 & GlpE \\
\hline 4140 & raxR \\
\hline 4141 & AvrXa21 \\
\hline 4142 & batB \\
\hline 4143 & sigW \\
\hline 4144 & retinol \\
\hline 4145 & $\mathrm{SCP}$ \\
\hline 4146 & $\mathrm{SbcCD}$ \\
\hline 4147 & coadecarboxylase \\
\hline 4148 & $\mathrm{XadA}$ \\
\hline 4149 & conditioned \\
\hline 4150 & density \\
\hline 4151 & $36 \mathrm{H}$ \\
\hline 4152 & sigma28 \\
\hline 4153 & hap3 \\
\hline 4154 & wece \\
\hline 4155 & CipB \\
\hline 4156 & inclusion \\
\hline
\end{tabular}

$-0.0030868$

$-0.0030868$

$-0.0030868$

$-0.0030868$

$-0.0030868$

$-0.0030868$

$-0.0030868$

$-0.0030868$

$-0.0030868$

$-0.0030868$

$-0.0030868$

$-0.0030868$

$-0.0030868$

$-0.0030868$

$-0.0030868$

$-0.0030868$

$-0.0030868$

$-0.0030868$

$-0.0030868$

$-0.0030868$

$-0.0030868$

$-0.0030868$

$-0.0030868$

$-0.0030868$

$-0.0030868$

$-0.0030868$

$-0.0030868$

$-0.0030868$

$-0.0030868$

$-0.0030868$

$-0.0030868$

$-0.0030868$

$-0.0030868$

$-0.0030868$

$-0.0030868$

$-0.0030868$

$-0.0030868$

$-0.0030868$

$-0.0030868$

$-0.0030868$

$-0.0030868$

$-0.0030868$

$-0.0030868$

$-0.0030868$

$-0.0030868$

$-0.0030868$

$-0.0030868$

$-0.0030868$

$-0.0030868$

$-0.0030868$

$-0.0030868$

$-0.0030868$

$-0.0030868$

$-0.0030868$ 


\begin{tabular}{|c|c|}
\hline 4157 & $54 \mathrm{~K}$ \\
\hline 4158 & NPr \\
\hline 4159 & harvesting \\
\hline 4160 & PBSX \\
\hline 4161 & nonfunctional \\
\hline 4162 & TM2 \\
\hline 4163 & domin \\
\hline 4164 & cycloartenol \\
\hline 4165 & Trax \\
\hline 4166 & OprM \\
\hline 4167 & IS3525 \\
\hline 4168 & DMSO \\
\hline 4169 & IS1548 \\
\hline 4170 & NirV \\
\hline 4171 & haloperoxidase \\
\hline 4172 & Toxs \\
\hline 4173 & TOxR \\
\hline 4174 & CitG \\
\hline 4175 & Luxs \\
\hline 4176 & ScpA \\
\hline 4177 & BtpA \\
\hline 4178 & PCXA \\
\hline 4179 & enol \\
\hline 4180 & $\operatorname{arcA}$ \\
\hline 4181 & autoagglutination \\
\hline 4182 & $\mathrm{cbiO}$ \\
\hline 4183 & CbiQ \\
\hline 4184 & YloV \\
\hline 4185 & Octanoyltransferase \\
\hline 4186 & OprD \\
\hline 4187 & Ahal \\
\hline 4188 & HyaE2 \\
\hline 4189 & SotB \\
\hline 4190 & blue \\
\hline 4191 & AroB \\
\hline 4192 & LlpA1 \\
\hline 4193 & PcaK \\
\hline 4194 & Rex \\
\hline 4195 & YbhD \\
\hline 4196 & PaaI \\
\hline 4197 & oxydoreductase \\
\hline 4198 & ExOA \\
\hline 4199 & $\mathrm{HisZ}$ \\
\hline 4200 & frataxin \\
\hline 4201 & $\mathrm{HmCD}$ \\
\hline 4202 & $\mathrm{COP}$ \\
\hline 4203 & FtrA \\
\hline 4204 & Legionaminic \\
\hline 4205 & exposed \\
\hline 4206 & $\mathrm{BCCP}$ \\
\hline 4207 & GSPF \\
\hline 4208 & ISRSO10 \\
\hline 4209 & C14 \\
\hline 4210 & yir2 \\
\hline
\end{tabular}

$-0.0030868$

$-0.0030868$

$-0.0030868$

$-0.0030868$

$-0.0030868$

$-0.0030868$

$-0.0030868$

$-0.0030868$

$-0.0030868$

$-0.0030868$

$-0.0030868$

$-0.0030868$

$-0.0030868$

$-0.0030868$

$-0.0030868$

$-0.0030868$

$-0.0030868$

$-0.0030868$

$-0.0030868$

$-0.0030868$

$-0.0030868$

$-0.0030868$

$-0.0030868$

$-0.0030868$

$-0.0030868$

$-0.0030868$

$-0.0030868$

$-0.0030868$

$-0.0030868$

$-0.0030868$

$-0.0030868$

$-0.0030868$

$-0.0030868$

$-0.0030868$

$-0.0030868$

$-0.0030868$

$-0.0030868$

$-0.0030868$

$-0.0030868$

$-0.0030868$

$-0.0030868$

$-0.0030868$

$-0.0030868$

$-0.0030868$

$-0.0030868$

$-0.0030868$

$-0.0030868$

$-0.0030868$

$-0.0030868$

$-0.0030868$

$-0.0030868$

$-0.0030868$

$-0.0030868$

$-0.0030868$ 


\begin{tabular}{|c|c|}
\hline 4211 & EIIABC \\
\hline 4212 & Ner \\
\hline 4213 & $\mathrm{HlyC}$ \\
\hline 4214 & ribitol \\
\hline 4215 & hemoprotein \\
\hline 4216 & TssM \\
\hline 4217 & syringe \\
\hline 4218 & needle \\
\hline 4219 & TssI \\
\hline 4220 & $\mathrm{PA} 2$ \\
\hline 4221 & PapA2 \\
\hline 4222 & IscR \\
\hline 4223 & $44 \mathrm{H}$ \\
\hline 4224 & bidirectional \\
\hline 4225 & diaphorase \\
\hline 4226 & $\mathrm{Cbc} 6$ \\
\hline 4227 & SPOR \\
\hline 4228 & Ykud \\
\hline 4229 & TssG \\
\hline 4230 & TssH \\
\hline 4231 & PP2C \\
\hline 4232 & articulin \\
\hline 4233 & FrpC \\
\hline 4234 & MycP1 \\
\hline 4235 & $\mathrm{RdgC}$ \\
\hline 4236 & MraW \\
\hline 4237 & LPPG \\
\hline 4238 & rbsc \\
\hline 4239 & AcrR \\
\hline 4240 & killer \\
\hline 4241 & ectoine \\
\hline 4242 & aminocyclopropane \\
\hline 4243 & Card \\
\hline 4244 & prevent \\
\hline 4245 & chlorite \\
\hline 4246 & formamidase \\
\hline 4247 & stationary \\
\hline 4248 & phase \\
\hline 4249 & CTERM \\
\hline 4250 & Fic \\
\hline 4251 & SLT \\
\hline 4252 & FliJ \\
\hline 4253 & reverse \\
\hline 4254 & transhydrogenase \\
\hline 4255 & partitioning \\
\hline 4256 & Cobyrinic \\
\hline 4257 & acylaminoacyl \\
\hline 4258 & сур141 \\
\hline 4259 & 141 \\
\hline 4260 & Hsp3 3 \\
\hline 4261 & subtype \\
\hline 4262 & S41 \\
\hline 4263 & different \\
\hline 4264 & $\mathrm{HIT}$ \\
\hline
\end{tabular}

$-0.0030868$

$-0.0030868$

$-0.0030868$

$-0.0030868$

$-0.0030868$

$-0.0030868$

$-0.0030868$

$-0.0030868$

$-0.0030868$

$-0.0030868$

$-0.0030868$

$-0.0030868$

$-0.0030868$

$-0.0030868$

$-0.0030868$

$-0.0030868$

$-0.0030868$

$-0.0030868$

$-0.0030868$

$-0.0030868$

$-0.0030868$

$-0.0030868$

$-0.0030868$

$-0.0030868$

$-0.0030868$

$-0.0030868$

$-0.0030868$

$-0.0030868$

$-0.0032228$

$-0.0032353$

$-0.0032353$

$-0.0032353$

$-0.0032353$

$-0.0032353$

$-0.0032364$

$-0.0034018$

$-0.0034311$

$-0.0034311$

$-0.0034311$

$-0.0034311$

$-0.0034311$

$-0.0034311$

$-0.0034311$

$-0.0036754$

$-0.0036754$

$-0.0036911$

$-0.0037191$

$-0.0037191$

$-0.0037191$

$-0.0037191$

$-0.0037191$

$-0.0037191$

$-0.0037191$

$-0.0037191$ 


\begin{tabular}{|c|c|}
\hline 4265 & L24 \\
\hline 4266 & patch \\
\hline 4267 & NhaA \\
\hline 4268 & dithiol \\
\hline 4269 & OPT \\
\hline 4270 & plasma \\
\hline 4271 & CheB \\
\hline 4272 & CflA \\
\hline 4273 & RsbU \\
\hline 4274 & ketosteroid \\
\hline 4275 & YbjL \\
\hline 4276 & YidE \\
\hline 4277 & arylformamidase \\
\hline 4278 & $\mathrm{Pi}$ \\
\hline 4279 & $\operatorname{cin} A$ \\
\hline 4280 & $\mathrm{BON}$ \\
\hline 4281 & YsxC \\
\hline 4282 & HipA \\
\hline 4283 & $\mathrm{C} 26$ \\
\hline 4284 & nodulation \\
\hline 4285 & mycosin \\
\hline 4286 & M4 2 \\
\hline 4287 & active \\
\hline 4288 & DAK2 \\
\hline 4289 & ech \\
\hline 4290 & $3 S$ \\
\hline 4291 & HisF \\
\hline 4292 & GFA \\
\hline 4293 & $1 \mathrm{C}$ \\
\hline 4294 & DprA \\
\hline 4295 & pyranopterin \\
\hline 4296 & TctC \\
\hline 4297 & helper \\
\hline 4298 & DUF1555 \\
\hline 4299 & main \\
\hline 4300 & $\operatorname{Mg} 2$ \\
\hline 4301 & MOSC \\
\hline 4302 & PqqB \\
\hline 4303 & LamB \\
\hline 4304 & ricin \\
\hline 4305 & lectin \\
\hline 4306 & minor \\
\hline 4307 & AbrB \\
\hline 4308 & ester \\
\hline 4309 & mota \\
\hline 4310 & exbb \\
\hline 4311 & tolq \\
\hline 4312 & autotransporter \\
\hline 4313 & water \\
\hline 4314 & PcrA \\
\hline 4315 & Alginate \\
\hline 4316 & bond \\
\hline 4317 & phenol \\
\hline 4318 & WecB \\
\hline
\end{tabular}

$-0.0037191$

$-0.0037191$

$-0.0037191$

$-0.0037191$

$-0.0037191$

$-0.0037191$

$-0.0037191$

$-0.0037191$

$-0.0037191$

$-0.0037191$

$-0.0037191$

$-0.0037191$

$-0.0037191$

$-0.0037191$

$-0.0037191$

$-0.0037191$

$-0.0037191$

$-0.0037191$

$-0.0037191$

$-0.0037191$

$-0.0037191$

$-0.0037191$

$-0.0037191$

$-0.0037191$

$-0.0037191$

$-0.0037191$

$-0.0037191$

$-0.0037191$

$-0.0037191$

$-0.0037191$

$-0.0037191$

$-0.0037191$

$-0.0037191$

$-0.0037191$

$-0.0037191$

$-0.0037632$

$-0.0037632$

$-0.0037632$

$-0.0037632$

$-0.0037632$

$-0.0037632$

$-0.0037632$

$-0.0037632$

$-0.0037632$

$-0.0038832$

$-0.0038832$

$-0.0038832$

$-0.0038832$

$-0.0038884$

$-0.0038884$

$-0.0038884$

$-0.0038884$

$-0.0040211$

$-0.0040408$ 


\begin{tabular}{|c|c|}
\hline 4319 & seryl \\
\hline 4320 & CpsF \\
\hline 4321 & aldose \\
\hline 4322 & carnitine \\
\hline 4323 & $\mathrm{~s} 11$ \\
\hline 4324 & VacJ \\
\hline 4325 & MucR \\
\hline 4326 & IM30 \\
\hline 4327 & DL \\
\hline 4328 & ribokinase \\
\hline 4329 & Folate \\
\hline 4330 & YgfZ \\
\hline 4331 & Met \\
\hline 4332 & death \\
\hline 4333 & extradiol \\
\hline 4334 & outermembrane \\
\hline 4335 & SpeA \\
\hline 4336 & PCS \\
\hline 4337 & NreB \\
\hline 4338 & DD \\
\hline 4339 & steroid \\
\hline 4340 & RibF \\
\hline 4341 & IS256 \\
\hline 4342 & prime \\
\hline 4343 & dbpA \\
\hline 4344 & WhiE \\
\hline 4345 & F390 \\
\hline 4346 & enoate \\
\hline 4347 & mycelium \\
\hline 4348 & aerial \\
\hline 4349 & sialoglycoprotein \\
\hline 4350 & monomethyl \\
\hline 4351 & Carotenoid \\
\hline 4352 & L5 \\
\hline 4353 & RuvA \\
\hline 4354 & SseB \\
\hline 4355 & choloyL \\
\hline 4356 & SET \\
\hline 4357 & infection \\
\hline 4358 & abortive \\
\hline 4359 & $\mathrm{~d} 1$ \\
\hline 4360 & $\mathrm{RecN}$ \\
\hline 4361 & ISXoo2 \\
\hline 4362 & layer \\
\hline 4363 & ISPsy6 \\
\hline 4364 & molydopterin \\
\hline 4365 & WD4 0 \\
\hline 4366 & ispsy11 \\
\hline 4367 & sheath \\
\hline 4368 & AhpD \\
\hline 4369 & CAIB \\
\hline 4370 & $\operatorname{RecF}$ \\
\hline 4371 & Mraz \\
\hline 4372 & DRTGG \\
\hline
\end{tabular}

$-0.0040408$

$-0.0042027$

$-0.0042066$

$-0.0042294$

$-0.0042912$

$-0.0042912$

$-0.0042912$

$-0.0042912$

$-0.0042912$

$-0.0042912$

$-0.0042912$

$-0.0042912$

$-0.0043456$

$-0.0043456$

$-0.0043456$

$-0.0043456$

$-0.0043657$

$-0.0043657$

$-0.0043657$

$-0.0043657$

$-0.0043657$

$-0.0043657$

$-0.0043657$

$-0.0043657$

$-0.0043657$

$-0.0043657$

$-0.0043657$

$-0.0043657$

$-0.0043657$

$-0.0043657$

$-0.0043657$

$-0.0043657$

$-0.0043657$

$-0.0043657$

$-0.0043657$

$-0.0043657$

$-0.0043657$

$-0.0043657$

$-0.0043657$

$-0.0043657$

$-0.0043657$

$-0.0043657$

$-0.0043657$

$-0.0043657$

$-0.0043657$

$-0.0043657$

$-0.0043657$

$-0.0043657$

$-0.0043657$

$-0.0043657$

$-0.0043657$

$-0.0043657$

$-0.0043657$

$-0.0043657$ 


\begin{tabular}{|c|c|}
\hline 4373 & Bvgs \\
\hline 4374 & HTR \\
\hline 4375 & DEXX \\
\hline 4376 & MarC \\
\hline 4377 & 12 \\
\hline 4378 & $\mathrm{PA}$ \\
\hline 4379 & Pilz \\
\hline 4380 & diamide \\
\hline 4381 & indolepyruvate \\
\hline 4382 & cyclo \\
\hline 4383 & triad \\
\hline 4384 & ResB \\
\hline 4385 & CcmA \\
\hline 4386 & $\mathrm{MCP} s$ \\
\hline 4387 & IS1328 \\
\hline 4388 & IS1533 \\
\hline 4389 & IS111A \\
\hline 4390 & cutinase \\
\hline 4391 & CHASE \\
\hline 4392 & IS630 \\
\hline 4393 & ComEC \\
\hline 4394 & $\operatorname{Rec} 2$ \\
\hline 4395 & WhiB \\
\hline 4396 & $\mathrm{~b} 561$ \\
\hline 4397 & teichoic \\
\hline 4398 & CobB \\
\hline 4399 & reacting \\
\hline 4400 & $\mathrm{DMT}$ \\
\hline 4401 & trypsin \\
\hline 4402 & CobQ \\
\hline 4403 & coat \\
\hline 4404 & sites \\
\hline 4405 & G2445 \\
\hline 4406 & SpoIIIE \\
\hline 4407 & recr \\
\hline 4408 & mitochondrial \\
\hline 4409 & Gingipain \\
\hline 4410 & crossover \\
\hline 4411 & endodeoxyribonuclease \\
\hline 4412 & amylase \\
\hline 4413 & Fur \\
\hline 4414 & thiouridylate \\
\hline 4415 & $\mathrm{P} 60$ \\
\hline 4416 & typically \\
\hline 4417 & TagA \\
\hline 4418 & LysM \\
\hline 4419 & subtilisin \\
\hline 4420 & Muts \\
\hline 4421 & RuvC \\
\hline 4422 & $\operatorname{Rec} Q$ \\
\hline 4423 & autolysis \\
\hline 4424 & globin \\
\hline 4425 & mutator \\
\hline 4426 & MinD \\
\hline
\end{tabular}

$-0.0043657$

$-0.0043657$

$-0.0043657$

$-0.0043657$

$-0.0044498$

$-0.0045761$

$-0.0045761$

$-0.0046907$

$-0.0048029$

$-0.0048192$

$-0.0048192$

$-0.0048192$

$-0.0048192$

$-0.0048192$

$-0.0048192$

$-0.0048192$

$-0.0048192$

$-0.0048192$

$-0.0048192$

$-0.0048192$

$-0.0048192$

$-0.0048192$

$-0.0048192$

$-0.0048192$

$-0.0048192$

$-0.0048192$

$-0.0048588$

$-0.0052602$

$-0.0052602$

$-0.0052602$

$-0.0052678$

$-0.0053471$

$-0.0053471$

$-0.0053471$

$-0.0053471$

$-0.0053471$

$-0.0053471$

$-0.0053471$

$-0.0053471$

$-0.0054172$

$-0.0055002$

$-0.0056768$

$-0.0056963$

$-0.0057174$

$-0.0057174$

$-0.0057174$

$-0.0057497$

$-0.0061747$

$-0.0061747$

$-0.0061747$

$-0.0061747$

$-0.0064411$

$-0.0064411$

$-0.006443$ 
4427 formaldehyde

4428 auxin

4429 MotB

4430 OmpA

4431 GDP

4432 motif

4433 nitroreductase
$-0.0066574$

$-0.0067938$

$-0.0068666$

$-0.0072839$

$-0.0074782$

$-0.0077608$

$-0.0080366$ 